\title{
EXAMINING HEALTH ANXIETY AMONG LYNCH SYNDROME CARRIERS
}

\author{
by \\ Jenna J. Albiani \\ Master of Arts, Ryerson University, 2009 \\ Bachelor of Arts, Wilfrid Laurier University, 2007
}

\author{
A dissertation \\ presented to Ryerson University \\ in partial fulfillment of the \\ requirements for the degree of \\ Doctor of Philosophy \\ in the Program of \\ Psychology
}

Toronto, Ontario, Canada, 2014

(C) Jenna Albiani 2014 


\section{Author's Declaration for Electronic Submission of a Dissertation}

I hereby declare that I am the sole author of this dissertation. This is a true copy of the dissertation, including any required final revisions, as accepted by my examiners.

I authorize Ryerson University to lend this dissertation to other institutions or individuals for the purpose of scholarly research.

I further authorize Ryerson University to reproduce this dissertation by photocopying or by other means, in total or in part, at the request of other institutions or individuals for the purpose of scholarly research.

I understand that my dissertation may be made electronically available to the public. 


\title{
Examining Health Anxiety among Lynch Syndrome Carriers \\ Doctor of Philosophy, 2014
}

\author{
Jenna J. Albiani \\ Psychology Program \\ Ryerson University
}

\begin{abstract}
The current research was designed to examine health anxiety among individuals with Lynch syndrome; a genetic predisposition to adult onset cancers. This research had two aims: 1) To examine the severity of health anxiety in Lynch syndrome patients and identify predictors and consequences associated with health anxiety, and 2) To examine the additional impact health anxiety has on parents with Lynch syndrome. Two studies were conducted. In Study I, 209 individuals with Lynch syndrome, selected from a genetic cancer registry, completed self-report measures assessing health anxiety, medical and psychological variables, and medical service utilization. Results indicated that $30 \%$ of participants reported clinically significant levels of health anxiety. Regression analyses revealed that younger age, greater depression, anxiety, worry interference and emotional preoccupation coping were predictive of increased health anxiety. Increased health anxiety was associated with greater overall medical service utilization; specifically, visits to gastroenterologists and emergency departments. In Study II, purposive sampling was used to identify parents from Study I who reported the highest and lowest health anxiety. Twenty-one individuals completed semi-structured telephone interviews about their experience of being a parent with Lynch syndrome, their concerns of potentially passing down the genetic mutation to their children, and their perceptions
\end{abstract}


of their children's health. Qualitative content analysis using a template coding approach was used to examine the differences between parents with high and low health anxiety. Findings revealed that the most prevalent difference was in relation to parent's perceptions of their personal health. Those with high health anxiety experienced worries that were more extreme, demonstrated a hypervigilance towards physical symptoms, discussed the emotional and psychological consequences of Lynch syndrome as more negative and severe, and had a tendency to engage in more dysfunctional coping strategies. Unexpectedly, with regards to their perceptions of their children, the parents in the high and low health anxiety groups exhibited similar worries. Taken together, the findings from Studies I and II suggest that health anxiety is of clinical significance for individuals with Lynch syndrome. Accurately identifying and treating health anxiety among this population may be one avenue to reduce the distress experienced by Lynch syndrome carriers. 


\section{Acknowledgements}

This project would not have been possible without the support and encouragement of many individuals. First and foremost, I would first like to express my sincerest thanks to my advisor, Dr. Tae Hart, for continually offering me the insight, guidance, time, and mentorship that have been instrumental in every step of my graduate pursuits. I would like to express my appreciation to my supervisory committee member, Dr. Kelly McShane, whose guidance and expertise have helped to strengthen this project. In addition, I would like to thank the additional members of my examining committee, Dr. Stephanie Cassin, Dr. Faith Donald and Dr. Margo Watt for agreeing to take time out of their busy schedules to review my dissertation and participate in my defense. I would also like to thank the amazing members of the Psychosocial Medicine Lab, particularly Lindsey Torbit and Noemi Katona, for their participation in this research.

Very special thanks are extended to the extraordinary Mount Sinai Hospital Zane Cohen Centre for Digestive Diseases Team for their support of this project and assistance with data collection.

For financial support, I thank the Canadian Institutes of Health Research.

I would also like to thank my wonderful classmates for their helpful advice, support, and friendship over the years. Finally, I would like to thank all of my family and friends for supporting my ambitions. I would especially like to thank my husband, Matthew.

Without your love, encouragement, and patience, none of this would have been possible. 
Dedication

To my mom. 


\section{Table of Contents}

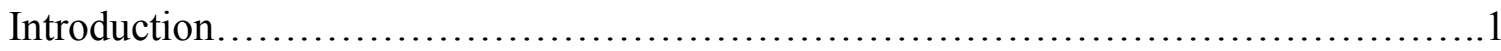

An Introduction to Lynch Syndrome..........................................

Psychological Reactions to Genetic Testing for Lynch Syndrome................2

Critiques of the Current Status of the Literature Examining Psychological

Functioning of Lynch Syndrome Carriers................................... 4

An Introduction to Health Anxiety.........................................

Health Anxiety: The Conceptual Model......................................7

Health Anxiety in Lynch Syndrome Carriers................................... 8

Health Anxiety in Medical Populations......................................8

Health Anxiety in Individuals at Risk for Developing HBOC....................10

Health Anxiety in Cancer Patients..........................................11

Predictors of Health Anxiety.............................................12

Consequences of Health Anxiety............................................ 14

Health Anxiety: The Additional Impact on Parents with Lynch Syndrome.......15

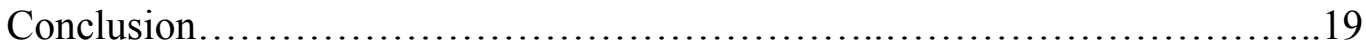

Study Aims and Hypotheses.............................................20

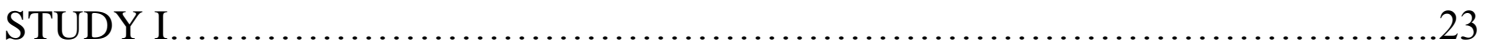

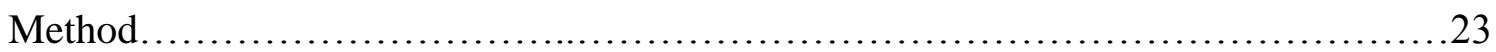

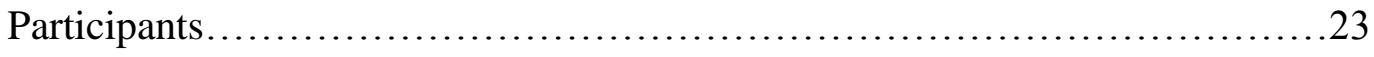

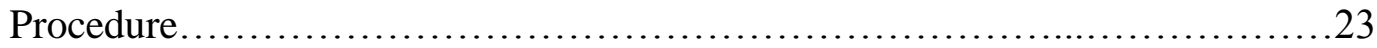

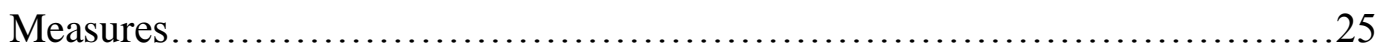

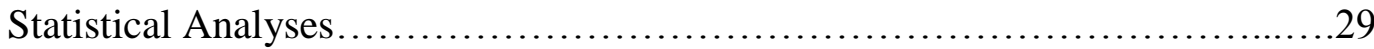


Results.

Preliminary Analyses................................................ 31

Sample Characteristics.............................................. 32

Descriptive Information on Key Variables..............................4 41

Hypothesis Testing .................................................44

Severity of Health Anxiety among Lynch Syndrome Carriers.............44

Predictors of Health Anxiety....................................48

Consequences of Health Anxiety.................................50

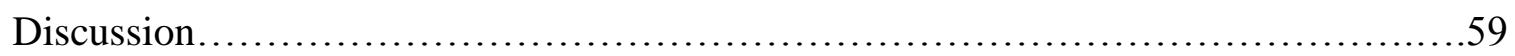

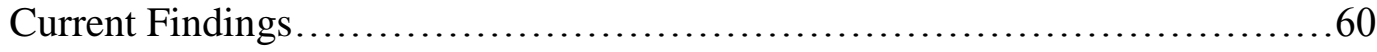

Severity of Health Anxiety among Lynch Syndrome Carriers............60

Predictors of Health Anxiety...................................65

Consequences of Health Anxiety.................................73

Limitations of the Present Study..................................... 77

STUDY II............................................................... 81

Method.................................................................. 81

Research Approach.................................................... 81

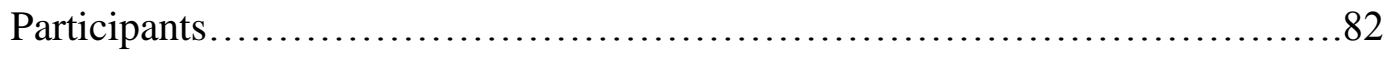

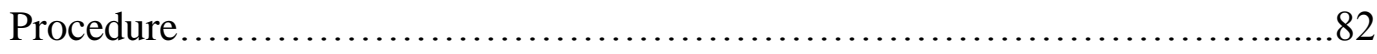

Qualitative Analysis................................................84

Results.................................................................. 86

Sample Characteristics............................................... 86 
Overview

Main Findings................................................... 90

Domain 1: Perceptions of Self.................................90

Domain 2: Perceptions of Passing Down the Gene to Child.............101

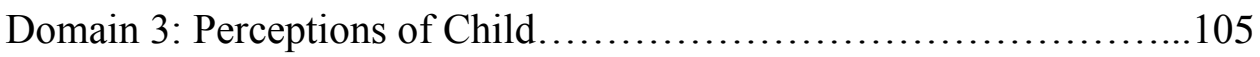

Discussion............................................................... 112

Overall Similarities and Differences between High and Low Health

Anxious Parents.....................................................112

The Impact of Health Anxiety on Perceptions of Self........................113

The Impact of Health Anxiety on Perceptions of Passing Down Lynch

Syndrome to Child...................................................... 117

The Impact of Health Anxiety on Perceptions of Children's Health.............121

Limitations of the Present Study...................................... 125

DISCUSSION AND INTEGRATION OF FINDINGS FROM STUDIES I AND II....128

Clinical Implications............................................. 132

Future Directions....................................................... 134

Appendices............................................................ 136

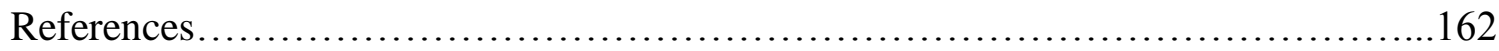




\section{List of Tables}

Table 1: Correlation Matrix of Key Psychological Variables...........................33

Table 2: Study I Sample Demographics.................................... 35

Table 3: Study I Medical Characteristics..................................... 38

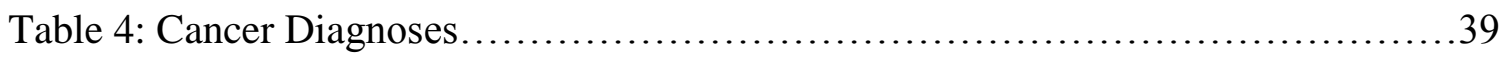

Table 5: Means, Standard Deviations, and Ranges for Key Variables..................42

Table 6: Medical Visits over the Past Six Months.................................46

Table 7: Frequency of Severe and High Health Anxiety Based on the Health

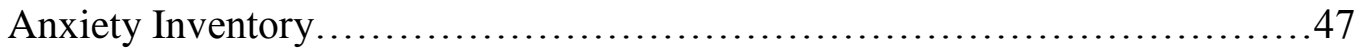

Table 8: Hypothesis 2a: Multiple Regression Analysis of Demographic and Medical Predictors of Health Anxiety.............................................49

Table 9: Hypothesis 2b: Psychological Predictors of Health Anxiety..................51

Table 10: Hypothesis 3: Consequences of Health Anxiety $\ldots \ldots \ldots \ldots \ldots \ldots \ldots \ldots \ldots \ldots . . .53$

Table 11: Study II Sample Demographics and Medical Characteristics.................88

Table 12: Demographic and Medical Information about the Children of Study II Participants.......................................................91 


\section{List of Figures}

Figure 1: Visual Representation of the Study II Coding Scheme.......................87 


\section{List of Appendices}

Appendix A: Study I Questionnaire Package..................................136

Appendix B: Study II Semi-Structured Interview Guide........................153

Appendix C: Study II Codebook..............................................156 


\section{Examining Health Anxiety among Lynch Syndrome Carriers}

\section{An Introduction to Lynch Syndrome}

Lynch syndrome, also known as hereditary nonpolyposis colorectal cancer (HNPCC), is a genetic condition characterized by a predisposition to adult-onset cancers. Lynch syndrome is caused by mutations in DNA mismatch repair genes (i.e., MLH1, MSH2, MSH6, and PMS2) and affects men and women at equal rates (Järvinen et al., 2009; Järvinen et al., 2000; Lindor et al., 2006). Although patients with Lynch syndrome most commonly develop colorectal cancer, they are also at increased risk for developing cancers of the endometrium, ovaries, stomach, small intestine, hepatobiliary system, pancreas, ureter, kidney and brain (Lindor et al., 2006; Lynch et al., 2009; Möslein, Krause-Paulus, Hegger, Peterschulte, \& Vogel, 2000). Lynch syndrome related cancers occur at younger ages, with Lynch syndrome-associated tumors developing approximately 20 years earlier than sporadic (i.e., non-hereditary) tumors. As such, the average age for tumor development for colorectal and gynecological cancers is between 45 and 50 years of age (Mitchell, Farrington, Dunlop, \& Campbell, 2002). Furthermore, Lynch syndrome cancers progress more rapidly than the same cancers seen in the general population (Lindor et al., 2006).

Lynch syndrome accounts for approximately $3 \%$ of all colorectal cancer occurrences (Hampel et al., 2008), which translates to approximately 36,000 annual cases worldwide (Jemal et al., 2011). In the absence of cancer screening, it is estimated that the cumulative risk of developing any Lynch syndrome-related cancers by age 70 is between 47 and 85 percent (Jenkins et al., 2006). More specifically, individuals with Lynch syndrome have an 80 percent chance of developing colorectal cancer in their lifetime and, 
for women with Lynch syndrome, the estimated lifetime risk of endometrial cancer is 40 to 60 percent (Aarnio et al., 1999; Lindor et al., 2006). The significance of these lifetime risk estimates is highlighted when these rates are compared to those of the general population. Specifically, for individuals without genetic mutations, the lifetime risk of developing colorectal cancer and endometrial cancer is approximately five percent and two percent, respectively (Siegel, Naishadham, \& Jemal, 2012).

Lynch syndrome is passed on through families in an autosomal dominant inheritance pattern, meaning that if one parent carries a genetic mutation for Lynch syndrome, there is a 50 percent chance that the mutation will be inherited by each child (Lynch et al., 2009). Given this inheritance pattern, genetic testing for Lynch syndrome is suggested for members of high-risk families. If an individual receives confirmation that they are a Lynch syndrome carrier, she or he is provided with information regarding the increased risk of developing cancer and are also counselled on the behavioural, medical and surgical options available to decrease cancer risk. As one can imagine, receiving knowledge of a hereditary predisposition to cancer can be a distressing event.

\section{Psychological Reactions to Genetic Testing for Lynch Syndrome}

Since the commencement of the clinical availability of genetic testing, concerns have been raised of the potential harmful emotional impact testing may have on individuals (Lerman \& Croyle, 1994; Rolland \& Williams, 2005). Although the body of research examining the emotional impact of testing for Lynch syndrome is somewhat scarce, overall, it appears that the majority of individuals who have undergone genetic testing for Lynch syndrome do not experience adverse psychosocial consequences over the long-term (e.g., Claes et al., 2005; Gritz et al., 2005; Meiser et al., 2004; Wagner et 
al., 2005). Nearly all individuals who have undergone genetic testing for Lynch syndrome report that they are satisfied with their decision to have done so (Arver, Haegermark, Platten, Lindblom, \& Brandberg, 2004; Esplen et al., 2001). Not surprisingly, following genetic testing, individuals who are deemed to be non-carriers of Lynch syndrome report decreases in cancer-specific distress, generalized anxiety, and depression. These reductions occur immediately following result disclosure and remain relatively stable over time (Aktan-Collan, Haukkala, Mecklin, Uutela, \& Kääriäinen, 2001; Claes et al., 2005; Claes, Denayer, Evers-Kiebooms, Boogaerts, \& Legius, 2004; Collins et al., 2007; Gritz et al., 2005; Meiser et al., 2004). For individuals who receive a positive mutation carrier result, generalized anxiety, cancer-specific distress and worry have been shown to increase in the period of time immediately post-diagnosis; however, these elevations tend to return to baseline levels within one year (Aktan-Collan et al., 2001; Claes et al., 2005, 2004; Collins et al., 2007; Gritz et al., 2005; Meiser et al., 2004). Within the genetic cancer literature, the majority of research examining distress levels among individuals being tested for cancer susceptibility has occurred in the context of predictive genetic testing for hereditary breast and ovarian cancer (HBOC). HBOC and Lynch syndrome share a number of commonalities. Specifically, similar to Lynch syndrome, HBOC is a genetic condition which is passed on through families in an autosomal dominant inheritance pattern. HBOC mutation carriers are at an increased risk of developing a variety of adult onset cancers, predominantly breast and ovarian cancer. The lifetime risk of breast cancer exceeds $80 \%$ and the lifetime risk of ovarian cancer is estimated to be up to $60 \%$ (Lancaster, Carney, \& Futreal, 1997). As such, the literature 
on HBOC can be used to inform our understanding of the experience of individuals living with Lynch syndrome.

In general, results from a decade of research examining the psychological impact of genetic testing for $\mathrm{HBOC}$ mirror the aforementioned findings reported by individuals being tested for Lynch syndrome. In particular, this body of research suggest that the vast majority of individuals do not experience long-term negative psychological effects after undergoing genetic testing for $\mathrm{HBOC}$, although many do report distress and worry when awaiting test results and upon receiving information that they carry a cancer-related genetic mutation (Broadstock, Michie, \& Marteau, 2000; Lerman, Croyle, Tercyak, \& Hamann, 2002).

\section{Critiques of the Current Status of the Literature Examining Psychological Functioning of Lynch Syndrome Carriers}

Despite the aforementioned positive or relatively benign impact of genetic testing for Lynch syndrome, it is important to note that there is a subset of individuals with genetic predispositions to cancer who do experience significant and persistent distress and cancer related worry (Broadstock, Michie, \& Marteau, 2000; Meiser \& Halliday, 2002; Wagner et al., 2005). The lack of overall elevated distress, however, has intrigued researchers in this area, some of whom have suggested that factors related to research design and methodology could be masking important aspects of the experience of living with a hereditary predisposition to cancer. One important critique of the literature is the limited amount of information on the psychological impact of being a Lynch syndrome carrier in the long-term. 
Currently, the majority of studies on Lynch syndrome carriers have focused on examining the psychological reactions of patients immediately following genetic testing. As such, prospective studies are generally designed to examine pre- and post-testing general psychological distress, typically with one year follow-up periods (e.g., Claes et al., 2005; Meiser et al., 2004). "Long-term" psychological functioning in this research has been examined by examining Lynch syndrome carriers 3.5 years after they received their testing results (Wagner et al., 2005). Although this research is commendable, it continues to focus on the effect of genetic testing and neglects the broader psychological experience being a Lynch syndrome carrier. Consequently, little is currently known about the impact of living with the knowledge that one is at an elevated risk to develop cancer.

Moreover, the majority of the current research presents findings for the entire sample group as a whole. The use of group statistics can conceal important individual differences within the data. Even if the sample as a whole reports a decrease or no significant reduction in distress with regards to receiving knowledge of Lynch syndrome carrier status, there may be a substantial minority of individuals who experience significantly negative reactions (Rimes, Salkovskis, Jones, \& Lucassen, 2006).

A final issue is the assessment of psychological functioning among those with Lynch syndrome. In particular, concerns have been raised that the measures are overly global and may have limited ability to identify specific psychosocial issues associated with carrying a hereditary cancer gene (Bleiker, Hahn, \& Aaronson, 2003; Esplen et al., 2009; Vadaparampil, Ropka, \& Stefanek, 2005). Findings from qualitative research and studies which have used more specific cancer risk measures support this idea, as they 
have noted more negative reactions to genetic testing, changes in self-perceptions and adjustment difficulties than is typically observed in quantitative research (Hallowell, Foster, Eeles, Ardern-Jones, \& Watson, 2004; Lim et al., 2004; Trask et al., 2001). The present study aims to address the aforementioned limitations by investigating the longterm impact of living with a genetic predisposition to cancer, the use of methodological procedures, such as in depth qualitative analyses, that have a higher degree of sensitivity to identify individual differences, and by examining a more specific psychological variable which may be impacting this population, namely, health anxiety.

\section{An Introduction to Health Anxiety}

One specific psychological factor which may be particularly relevant for individuals with Lynch syndrome is health anxiety. Given the high degree of cancer likelihood, the early onset of cancer, and fast rate of progression of the disease (Lindor et al., 2006; Lynch et al., 2009; Mitchell et al., 2002; Möslein et al., 2000), it is likely that Lynch syndrome carriers experience a heightened and persistent anxiety which is focused on the threat to their health. This focus on the threat to one's health, termed health anxiety, has yet to be examined within a Lynch syndrome population.

Health anxiety is characterized by fear or worry about ill health and exists along a continuum, ranging from a sense of indifference or inattentiveness to health to intense health-related concerns and continuous worry about bodily symptoms or sensations (Salkovskis \& Warwick, 1986). Mild levels of health anxiety can be adaptive, serving to motivate individuals to seek health care services in situations where medical service utilization is warranted (Taylor \& Asmundson, 2004). However, more severe levels of health anxiety has been shown to be problematic, leading to personal distress, impaired 
social and occupational functioning, and overutilization of health care services (Barsky, Ettner, Horsky, \& Bates, 2001; Barsky, Fama, Bailey, \& Ahern, 1998; Fink, Ørnbøl, \& Christensen, 2010; Sunderland, Newby, \& Andrews, 2012; Taylor \& Asmundson, 2004). In order to avoid unnecessary health care utilization and improve patient quality of life, researchers have suggested that individuals experiencing heightened health anxiety be identified and treated (Fink et al., 2010).

\section{Health Anxiety: The Conceptual Model}

The conceptual framework used to guide the present research was the cognitivebehavioural model of health anxiety (i.e., HA model) developed by Warwick and Salkovskis (1990). The HA model (Warwick \& Salkovskis, 1990) is currently regarded as the most comprehensive and well-tested approach to exploring and understanding health anxiety (Taylor \& Asmundson, 2004). According to the HA model, individuals with persistent health anxiety perceive harmless physical sensations and other health information to be threatening. These perceptions of threat trigger a cycle of interrelated factors including emotional distress, dysfunctional cognitions about illness, physiological arousal, and behaviours intended to reduce distress (e.g., body checking, reassurance seeking). These factors influence one another and serve to maintain and exacerbate the anxiety an individual experiences (Warwick \& Salkovskis, 1990). The extent to which an individual experiences health anxiety is determined by four key cognitions, namely; (1) the perceived likelihood of having or developing an illness; (2) the perceived severity of the illness; (3) the perceived ability to cope with the illness; and (4) the perceived adequacy of medical resources available for treating the illness (Hadjistavropoulos et al., 2012; Rimes et al., 2006; Warwick \& Salkovskis, 1990). 


\section{Health Anxiety in Lynch Syndrome Carriers}

Unfortunately, little is known about the degree of health anxiety experienced by individuals with Lynch syndrome. However, it has been suggested that people have an increased risk of experiencing health anxiety if they have a medical condition that is chronic, intermittent, degenerative, involves uncertainty, or has no clearly recognized cause (Furer, Walker, \& Freeston, 2001). As Lynch syndrome and its associated cancers fulfill a number of these criteria, it is likely that health anxiety is a relevant variable for individuals with Lynch syndrome. To gain a further understanding of the likelihood that health anxiety is a concern for Lynch syndrome carriers, it is also important to look to research investigating this construct in other medical populations.

\section{Health Anxiety in Medical Populations}

Within the general (i.e., healthy) population, the prevalence rate of high health anxiety is estimated to be approximately three to six percent (Bleichhardt \& Hiller, 2007; Sunderland et al., 2012). The basic proponents of the HA model suggest that individuals who are diagnosed with medical conditions are at an even higher risk of experiencing heightened health anxiety. Specifically, the HA model predicts that when individuals are exposed to health related information (e.g., physical sensations) their dysfunctional health related beliefs are triggered and, as a result, health anxiety escalates (Warwick \& Salkovskis, 1990). It follows then, that for individuals experiencing health problems, the frequency with which they are exposed to potentially triggering health related information is increased, thus enhancing the likelihood that they experience heightened health anxiety. 
The empirical research in this area is supportive of this claim. Across a wide variety of medical conditions, all with varied physical symptoms, health anxiety appears to be higher in individuals with medical conditions when compared to healthy controls. For example, a study by Rode and colleagues (2006) reported that individuals attending a chronic pain clinic demonstrated significantly higher levels of health anxiety than nonclinical controls with and without pain. Moreover, the percentage of individuals meeting criteria for high health anxiety was very high within the sample of chronic pain clinic attendees, with a conservative estimate of $51.1 \%$ meeting criteria for high health anxiety.

Within a study by Kehler and Hadjistavropoulos (2009), multiple sclerosis patients reported higher levels of health anxiety than an age-matched control group from the general population, with 25 percent of individuals with multiple sclerosis reporting high levels of health anxiety. Moreover, elevated health anxiety was found to be associated with increased generalized anxiety, depression, reassurance seeking, emotional preoccupation, and higher demands on social support systems. Similarly, in a study comparing Ménière's disease patients to healthy controls, individuals with Ménière's disease reported significantly higher levels of health anxiety than the control group (Kirby \& Yardley, 2009). Again, health anxiety was also associated with elevated levels of anxiety and depression in this sample. In line with this, individuals with vulvar vestibulitis syndrome also reported significantly higher health anxiety than individuals who experienced pain-free sexual intercourse (Payne et al., 2007). In comparing a sample of individuals with self-reported medical conditions to a group of healthy controls, Pugh et al. (2012) reported that the individuals with medical conditions reported 
higher levels of persistent health anxiety and greater doubt in their physicians than the individuals in the control group.

Among those with medical conditions, the degree of health anxiety can impact reactions to one's disease. For example, Ratcliffe, MacLeod, and Sensky (2006) found myocardial infarction patients with elevated health anxiety perceived themselves to be at a greater risk for having another myocardial infarction and displayed more body vigilance than patients with lower health anxiety. Additionally, in an investigation examining patient response to psychotherapy, it was found that chronic pain patients with higher levels of health anxiety experienced greater generalized anxiety, somatic sensations and catastrophic cognitions during therapy than those with lower levels of health anxiety (Hadjistavropoulos, Asmundson, LaChapelle, \& Quine, 2002). Hadjistavropoulous et al. (2012) compared health anxiety related cognitions between a healthy control sample and people who self-reported various medical conditions (i.e., multiple sclerosis, cancer, respiratory disease, arthritis, diabetes, thyroid conditions, heart disease, and kidney/liver disease). Results indicated that the cognitions involved in health anxiety differed to some extent between individuals with and without medical conditions. Specifically, individuals with self-reported medical conditions reported a greater perceived likelihood of developing an illness, greater perceived inability to cope with illness, and greater perceived inadequacy with medical services.

\section{Health Anxiety in Individuals at Risk for Developing HBOC}

Similar to the research on health anxiety in Lynch syndrome carriers, limited research has evaluated health anxiety among individuals at risk for developing HBOC. However, related constructs such as cancer worry, cancer-specific anxiety, and illness 
anxiety have been examined among this population. Research in this area suggests that a significant proportion of individuals who are undergoing genetic testing for $\mathrm{HBOC}$ or who are carriers of a genetic mutation predisposing them to $\mathrm{HBOC}$ experience heightened anxiety about their health. Watson and colleagues (1999), for example, found that $28 \%$ of a sample of women undergoing genetic testing for HBOC worried 'frequently or constantly' about breast cancer. Similarly, Trask and associates (2001)

found that approximately two-thirds of women who were high risk for developing breast cancer perceived their worries about breast cancer as interfering with their ability to function in a variety of domains in their life.

\section{Health Anxiety in Cancer Patients}

Elevated health anxiety has been frequently reported in individuals who have been diagnosed with cancer. Specifically, in a sample of women diagnosed with any stage of breast cancer over the past 18 months, 38 percent of women reported elevated levels of health anxiety (Grassi, Rossi, Sabato, Cruciani, \& Zambelli, 2004). Additionally, when surveyed at 6 and 12 weeks post-surgery, approximately 20 percent of women with early stage breast cancer report experiencing strong worries about their future health (Wade, Nehmy, \& Koczwara, 2004).

Moreover, levels of health anxiety appear to remain relatively consistent, even after a patient's cancer is in remission. For example, in a study examining breast and testicular cancer patients, Stark et al. (2004) found that one-third of the cancer patients in remission reported beliefs, concerns, and behaviours consistent with health anxiety. In a sample of long-term breast, colorectal, or prostate cancer survivors (i.e., > 5 years), specific cancer-related health anxiety was reported by a substantial proportion of the 
sample. Specific anxiety about cancer recurrence, fears that a physical symptom signifies a cancer recurrence, anxiety about developing a different type of cancer, and concerns about undergoing future diagnostic tests were reported by 26 to 44 percent of the sample (Deimling, Bowman, Sterns, Wagner, \& Kahana, 2005).

As the research reviewed above indicates, health anxiety is common in medical populations, such as those with chronic pain, cardiac disease, autoimmune diseases, and cancer (Hadjistavropoulos et al., 2012; Jones, Hadjistavropoulos, \& Sherry, 2012; Kehler \& Hadjistavropoulos, 2009; Ratcliffe et al., 2006). However, little is known about the degree of health anxiety experienced by individuals with Lynch syndrome. As such, examining health anxiety among this population is both warranted and important as health anxiety has been linked to distress, increased medical utilization, and decreased overall patient well-being (Barsky et al., 2001, 1998; Fink et al., 2010; Sunderland et al., 2012; Taylor \& Asmundson, 2004). Moreover, health anxiety is a clinically relevant variable to examine as it has been shown to be modifiable. Specifically, health anxiety can be effectively decreased through psychotherapy, particularly cognitive behavioural therapy (Greeven et al., 2007; Hedman et al., 2011; Seivewright et al., 2008; Warwick, Clark, Cobb, \& Salkovskis, 1996). To gain a more comprehensive assessment of health anxiety among Lynch syndrome carriers, an examination of predictors and consequences of health anxiety is necessary.

\section{Predictors of Health Anxiety}

Examinations of the predictors of health anxiety in cancer patients or individuals being tested for genetic susceptibility to cancer (e.g., HBOC) provide useful information on relevant factors for Lynch syndrome carriers. This line of research has identified a 
variety of demographic, medical, and psychological variables linked to increased anxiety about one's health. In particular, demographic variables such as being of a younger age, having a greater family history of cancer, losing a parent to cancer, and having younger children have been shown to be associated with increased anxiety about developing cancer (Jones et al., 2012; Rimes et al., 2006; Tilburt et al., 2011; I. Van Oostrom et al., 2006). Medical variables such as time since receiving a diagnosis of cancer, experiencing a previous major medical illness, and greater past exposure to prophylactic tests or treatments have also been demonstrated to increase patient anxiety (Jones et al., 2012). From a psychological perspective, increased anxiety about health in cancer populations and other medical groups has been shown to be significantly related to distress, depression and general anxiety (Hadjistavropoulos et al., 2012). Moreover, negative cognitive appraisals and increased perceived risk of cancer, decreased beliefs about the preventability of cancer, and increased perceptions about the severity of cancer have also been linked to health anxiety (Hadjistavropoulos et al., 2012). Health anxiety has also been associated with lower perceived ability to cope and the type of coping strategies individuals engage in, namely, greater use of emotional preoccupation and social support and less use of problem-focused coping (Hadjistavropoulos et al., 2012; Kehler \& Hadjistavropoulos, 2009).

Although not specific to health anxiety, research examining predictors of distress in individuals undergoing testing for Lynch syndrome can also serve as a guide to factors that may directly contribute to health anxiety among this population. Interestingly, there is a high degree of overlap between the aforementioned predictors of health anxiety in medical populations and predictors of general distress in Lynch syndrome patients. 
Specifically, being younger in age, female, having a greater family history of cancer, having lost a parent to cancer, having younger children, having increased exposure to cancer screening procedures, and having more negative beliefs about cancer, perceived risk, coping ability and perceived control of Lynch syndrome are factors correlated with increased distress within this population (d'Agincourt-Canning, 2005; Esplen et al., 2003; Gritz et al., 2005; Keller et al., 2002, 2008; Mcallister, 2002; Bettina Meiser, 2005; Rimes et al., 2006; Van Oostrom et al., 2006). Moreover, a number of these variables, particularly the psychological variables reflecting perceptions of cancer, perceived risk and coping ability, are consistent with the factors outlined in the HA model (Warwick \& Salkovskis, 1990). Taken together, the predictors of health anxiety in medical populations, the predictors of distress in Lynch syndrome patients, and the consistency these share with the HA model provide substantial support for examining similar variables as predictors of health anxiety in Lynch syndrome patients.

\section{Consequences of Health Anxiety}

Elevated health anxiety can have widespread ramifications; high levels of health anxiety can lead to personal distress, impaired social and occupational functioning, and overutilization of health care services (Barsky et al., 2001, 1998; Fink et al., 2010; Sunderland et al., 2012; Taylor \& Asmundson, 2004). Specific to health care utilization, high health anxiety has been shown to lead to greater total outpatient costs, more physician visits, and an increased likelihood of being hospitalized within a one year period as compared to individuals with lower levels of health anxiety in the general population (Barsky et al., 2001). Conroy and colleagues (1999) found that people with higher health anxiety engaged in an increased number of self-initiated physician visits in 
the previous year and also were more likely to believe that their problem would require investigation by a medical specialist. Bleichhart and Hiller (2007) found that high health anxiety was not only related to increased visits to the doctor, but also to increased use of psychotherapy and psychiatric treatment. Fink (2010) has argued that the health care costs of health anxiety alone provide reason enough to routinely investigate health anxiety. However, no data exist as to whether increased health anxiety in patients with Lynch syndrome is associated with greater medical service utilization. This is an aim of the proposed study.

\section{Health Anxiety: The Additional Impact on Parents with Lynch Syndrome}

Given that Lynch syndrome is a genetic disorder that is passed on through families in an autosomal dominant inheritance pattern (Lynch et al., 2009), if one parent carries this gene mutation, there is a 50 percent chance that the mutation will be inherited by each child. It is not surprising then, that concerns for one's child is at the forefront of the minds of parents who carry the Lynch syndrome mutation. In fact, parents consistently cite the desire to obtain cancer risk information for their children as the primary factor that motivated them to seek genetic testing (Claes et al., 2004; Esplen et al., 2001; Lerman, Marshall, Audrain, \& Gomez-Caminero, 1998; Patenaude et al., 2006). For example, in a study by Hadley and colleagues (2003) which investigated attitudes, intention, and completion of genetic testing among 111 first-degree relatives of individuals with Lynch syndrome, the majority of the sample indicated that learning about their children's risks was the most important reason to consider genetic testing.

Genetic testing for cancer syndromes impacts the entire family; however, the manner in which families are impacted is somewhat mixed. Van Oostrom and colleagues 
(2006) found that 37 percent of individuals undergoing genetic susceptibility testing for HBOC or Lynch syndrome reported that genetic testing affected their family relationships in a positive manner. For example, these individuals reported increased feelings of closeness and improved communication and support following genetic testing. In contrast, however, 19 percent of this same sample reported that they experienced unwanted changes in their family relationships such as increased feelings of guilt towards children, difficulties with communication, and imposed secrecy. Mixed findings in this regard suggest that additional psychological variables may be involved in this relationship. Parental levels of health anxiety may be one factor which explains the inconclusive findings in this area.

As posited by the HA model, individuals with high health anxiety have a tendency to misinterpret benign physical sensations as signs of disease, overestimate the probability of serious illness and perceive physical symptoms to be more dangerous than they actually are (Warwick \& Salkovskis, 1990). Although the HA model is focused on factors that trigger an individual's anxiety about their own health, it is not unreasonable to believe that parents with heightened health anxiety could experience a similar process of hypervigilance and distorted cognitions in regards to their children's physical symptoms and health. As such, for Lynch syndrome carriers, the experience of being genetically predisposed to cancer likely takes on additional meaning for patients who are parents. Furthermore, this experience is likely worsened in parents who experience high levels of health anxiety. It appears as though there are at least three domains which are particularly relevant to parents who are Lynch syndrome carriers; 1) Perceptions of one's 
own health, 2) Psychological consequences of potentially passing down the genetic mutation to one's child; and 3) Perceptions about one's child's health.

Perceptions of own health and role as a parent. From the larger cancer literature, it has been shown that after receiving a diagnosis of cancer, patients who have children report additional anxiety, which tends to be related to their role as parents.

Being a parent seems to amplify the negative psychological consequences associated with cancer. For example, in a study comparing cancer patients who had children under the age of 18 to patients who did not, the patients with younger children were at an increased likelihood of developing an acute stress disorder or an anxiety disorder (Ernst, Götze, Brähler, Körner, \& Hinz, 2012; Krauß, Ernst, Kuchenbecker, Hinz, \& Schwarz, 2007). Moreover, parents who have been diagnosed with cancer report experiencing worries that are focused on their children and their role as parents. Predominant worries include concerns about their ability to care for their children if they become ill and anxiety about leaving children without a parent should they die from cancer (Walsh, Manuel, \& Avis, 2005). Although having a genetic predisposition to cancer (i.e., Lynch syndrome) is certainly not the same as being diagnosed with cancer, knowing that one is at an increased risk of developing cancer increases feelings of vulnerability and may cause parents to experience similar concerns about the effect having cancer in the future would have on their children.

\section{The experience of potentially passing down the genetic mutation to one's}

child. With regards to thoughts and feelings associated with passing down the genetic mutation to one's child, parents report experiencing a range of emotions. Parents undergoing genetic testing for cancer mutations frequently report feelings of guilt, 
depression, and anxiety when thinking about the possibility of having passed down the genetic mutation to their child (Bartuma, Nilbert, \& Carlsson, 2012; Murakami et al., 2004; Iris Van Oostrom et al., 2006). In fact, for a minority of men and women undergoing predictive genetic testing for genetic susceptibility to cancer, the concern about passing down the genetic mutation to their future children was so strong that individuals reported that the results of genetic testing could affect their family planning decisions (Bartuma, Nilbert, \& Carlsson, 2012; Lynch et al., 1997). Given that individuals experiencing high levels of health anxiety have a tendency to overestimate both the probability and the severity of illness (Warwick \& Salkovskis, 1990), it is likely that parents - when thinking about the possibility of their child being predisposed to developing cancer - will experience heightened negative emotional reactions.

Parental perceptions of one's child's health. Finally, parents with genetic predispositions to cancer may have altered perceptions of their child's health. Research has demonstrated that parents who are carriers of a genetic predisposition to cancer experience anxiety about their child's health and worry about their child developing cancer in the future (Bartuma et al., 2012; Gaff, Lynch, \& Spencer, 2006). For parents who have already been diagnosed with cancer, fears regarding their children's health may be further amplified. For example, in a study examining carriers of Lynch syndrome or HBOC who had a cancer diagnosis, $80 \%$ of the patients expressed concern over their children's future health. In particular, the patients reported concerns about their child carrying the cancer predisposing mutation and feared that their child would develop a form of cancer (Bonadona et al., 2002). Current guidelines for predictive genetic testing of cancer susceptibility suggest that such tests be made available to those aged 18 or 
older, unless there is clear medical benefit to testing earlier (Trepanier et al., 2004). Consequently, parents of young children and adolescents are unable to receive confirmation of their child's carrier status. For parents who experience high health anxiety, this ambiguity and uncertainty likely causes increased distress and promotes a hypervigilance towards their child's health. Moreover, for parents who have knowledge of an adult child's carrier status, they may be at an increased likelihood to misinterpret physical symptoms their adult child experiences as signs of cancer and overestimate the probability of their child developing cancer in the future.

Overall, it is evident that anxiety is at the forefront of a number of the concerns expressed by parents with genetic susceptibilities to cancer. Given this, it is likely that parents who have elevated health anxiety experience exacerbated negative reactions to the way they view their own health, their children's health, and their feelings about potentially passing down the genetic mutation. Therefore, parents with high levels of health anxiety may be a subgroup of individuals with Lynch syndrome who are particularly at risk for experiencing additional distress. Currently, the role that health anxiety plays in the experience of being a parent diagnosed with Lynch syndrome is unknown.

\section{Conclusion}

Although health anxiety has been well studied in a variety of medical populations, less is known about the health anxiety experienced by individuals diagnosed with Lynch syndrome. Presently, the majority of research examining the psychological effects of Lynch syndrome is focused on individuals' anxiety and distress at the time of genetic testing and diagnosis. To date, it is unclear the extent to which Lynch syndrome carriers 
experience health anxiety. Additionally, the factors which predict elevated health anxiety in Lynch syndrome patients is unknown, as are the consequences of being a Lynch syndrome carrier with high health anxiety. Furthermore, although parents with Lynch syndrome likely represent a subgroup of patients who are at risk for experiencing additional distress, the impact that health anxiety has on parental perceptions of one's own health and one's child's health has yet to be examined.

The present research addressed these deficits in the literature through four specific aims. This research aimed to: 1) Describe the severity of health anxiety among Lynch syndrome carriers, 2) Identify predictors of elevated health anxiety among Lynch syndrome carriers, 3) Identify consequences of elevated health anxiety among Lynch syndrome carriers, and 4) Explore the impact of health anxiety on the experience of being a parent who is a Lynch syndrome carrier.

\section{Study Aims and Hypotheses}

Aim 1. The first aim of Study I was to describe the severity of health anxiety among Lynch syndrome carriers. It was hypothesized that individuals with Lynch syndrome would describe a range of health anxiety levels. It was expected that a proportion would experience levels at or above the clinical cutoff.

Aim 2. The second aim of Study I was to identify predictors of elevated health anxiety among Lynch syndrome patients. Based on the HA model and the aforementioned research examining correlates of heightened health anxiety, it was hypothesized that:

a. Demographic and clinical factors would be associated with health anxiety. Specifically, health anxiety would be higher in patients who 
were female, younger, who had younger children, had previously been diagnosed with cancer, had more first degree relatives diagnosed with cancer, and who had been more recently diagnosed with LS.

b. Psychological factors would be associated with health anxiety. Specifically, increased levels of health anxiety would be predicted by increased use of emotion-focused coping strategies, decreased use of problem-focused coping strategies, higher depression, higher levels of anxiety, and higher levels of worry interference.

Aim 3. The third aim of Study I was to identify consequences of elevated health anxiety among Lynch syndrome patients. Based on the research examining correlates of heightened health anxiety, it was hypothesized that increased levels of health anxiety would be associated with higher levels of medical service utilization.

Aim 4. The purpose of Study II was to investigate the impact of health anxiety on the experience of being a parent who is a Lynch Syndrome carrier. Study II used a qualitative approach and was based on in-depth, semi-structured interviews conducted with a subset of the study participants. Study II sought to contrast the experience of parents who are Lynch Syndrome carriers representing both ends of the health anxiety continuum. The purpose of contrasting high versus low health anxiety participants was to qualitatively explore the differences between the two subgroups in:

4a. The experience of being a parent with Lynch syndrome. 
4b. The experience of potentially passing down the genetic mutation to one's child.

4c. The parental perceptions of their child's health and vulnerability to developing cancer.

The present research was comprised of a two studies. Study I was a crosssectional, self-report questionnaire-based study. Study II was qualitative in nature and based on interviews conducted with a subset of participants. Study I method, results, and discussion are presented first, followed by the Study II method, results, and discussion sections. Finally, an integration of the findings from Study I and II is offered. 


\section{STUDY I}

\section{Method}

\section{Participants}

Participants were recruited from the Familial Gastrointestinal Cancer Registry (FGICR), which is housed at Mount Sinai Hospital in Toronto, Canada. The FGICR, established in 1980, is a database comprised of patients and families at high risk of hereditary gastrointestinal cancers. Confirmation of the diagnosis of a hereditary predisposition to gastrointestinal cancers (e.g., Lynch syndrome) through medical records and testing is required for enrollment in the registry. The purpose of the FGICR is to study and understand a variety of variables related to inherited gastrointestinal cancer syndromes. As members of the FGICR, all participants have provided permission to be contacted for future research studies.

For patients to be included in Study I, the following criteria must have been met: 1) Be over 18 years of age. 2) Have provided consent to partake in the FGICR. 3) Have a diagnosis of Lynch syndrome (as confirmed by the FGICR). 4) Be able to speak and read English.

\section{Procedure}

Study I was based on a one-time self-report questionnaire. Participants in the FGICR were mailed a package containing an invitation to participate in the study, a consent form, and a questionnaire package (Appendix A). Participants were asked to return the signed consent form and the completed questionnaire package using the selfaddressed stamped return envelopes provided. The time required for completion of the questionnaire was approximately 60 minutes. As reimbursement for their time, 
participants were mailed a \$20.00 gift card upon receipt of their completed questionnaire package.

FGICR participants who did not return their questionnaire package within eight weeks of the original mail out date were telephoned by the research team to enquire about their intent to participate in the study. Participants who indicated that they were not interested in completing the study were not contacted further. Participants who stated that they intended to participate in the study were given an additional four weeks to return the questionnaire. If no questionnaire was received within this time frame, a second follow-up call was made to the individual as a reminder.

Response rate. In total, 424 questionnaires were mailed out to participants in the FGICR. However, 89 participants that received the initial mail out were unable to be contacted or were determined to be ineligible participants. Specifically, 14 packages were returned due to incorrect addresses and 56 individuals' telephone numbers were either unlisted or out of service. An additional 19 individuals were deemed ineligible by the research team. Reasons for ineligibility included death $(n=2)$, not having any contact with the FGICR over the past five years $(n=7)$, language difficulties $(n=3)$, previous requests to not be contacted for future studies $(n=2)$, or the genetic counsellors determining that the participant was medically or psychologically unfit to complete the study $(n=5)$. As such, the total number of eligible participants who received questionnaires was 335. Questionnaires were completed and returned by 209 individuals. Thus, the total response rate equaled $62.4 \%$. 


\section{Measures}

Medical utilization. Medical utilization was assessed via patient self-report, using items created for this study. Participants were asked to indicate how many times over the past six months they made visits to various health professionals. Participants were provided with a list of seven categories of health professionals (General Practitioner, Oncologist, Surgeon, Gastrointestinal Specialist, Cardiologist, Emergency Room Visit, Genetic Counsellor) and were also given space to add in an unlisted medical specialist. In addition to recording the number of visits made to each type of professional, participants were also asked to indicate if the appointment was a regularly scheduled check-up.

The Health Anxiety Inventory (short version). The Short Health Anxiety Inventory (SHAI; Salkovskis, Rimes, Warwick, \& Clark, 2002) is a validated self-report measure designed to assess the full continuum of health anxiety, ranging from low to severe health anxiety. The SHAI consists of 18 items closely aligned with the cognitive behavioural theory of health anxiety (Warwick \& Salkovskis, 1990). For each item, individuals were instructed to select one of four statements that most closely resembled their experience. For example, a participant was asked to select between the following statements: (1) I do not worry about my health. (2) I occasionally worry about my health. (3) I spend much of my time worrying about my health. (4) I spend most of my time worrying about my health. The SHAI was developed to minimize inflation of health anxiety scores in individuals who have an illness, making this scale appropriate for use among a Lynch syndrome population. The SHAI contains two subscales: 1) Illness 
Likelihood, and 2) Negative Consequences. ${ }^{1}$ The Illness Likelihood subscale contains 14-items and assesses the degree to which individuals are worried or preoccupied by a variety of bodily symptoms that are characteristic of severe health anxiety. The Negative Consequences subscale contains four items and compliments the Illness Likelihood subscale by assessing the degree to which participants perceive the negative consequences of being ill. The SHAI has been used to differentiate high versus normal levels of health anxiety. Specifically, a cutoff score of 18 or higher on the Illness Likelihood subscale has been used to identify individuals who meet criteria for severe health anxiety, whereas a score between 15 and 17 is representative of individuals with high health anxiety. Scores below 15 are considered to indicate normal levels of concern over one's health (Kehler \& Hadjistavropoulos, 2009; Rode, Salkovskis, Dowd \& Hanna, 2006; Seivewright et al., 2004). The SHAI demonstrates good internal consistency, with Cronbach's coefficient alpha equal to .89 in a sample of individuals diagnosed with hypochondriasis, panic disorder, social phobia, general practice clinic clients, medical outpatients and non-clinical controls (Salkovskis, Rimes, Warwick, \& Clark, 2002). Within the present study, the internal reliability of the SHAI was good (Cronbach's alpha $=.87)$.

The Hospital Anxiety and Depression Scale. The Hospital Anxiety and Depression Scale (HADS; Zigmond \& Snaith, 1983) is a self-report instrument for assessing anxiety and depression among non-psychiatric medical patients. This scale is

\footnotetext{
${ }^{1}$ Abramowitz, Deacon and Valentiner (2007) proposed that the SHAI contained three factors: 1) Perceived Likelihood of Illness, 2) Perceived Severity of Illness, and 3) Body Vigilance. More recently, however, Alberts and colleagues (2013) completed a systematic review and meta-analysis of research utilizing the SHAI and concluded that the original two-factor solution had the greatest support. As such, the current study elected to utilize the original two subscales.
} 
ideal for assessing anxiety and depression among medical patients because the HADS minimizes content related to physical symptoms associated with emotional disorders. The HADS has been used in hospital, outpatient, and community settings. The scale contains 14 items equally divided into two subscales: 1) The Anxiety Subscale (HADSA), and 2) The Depression Subscale (HADS-D). Each item is rated on a four-point scale with different verbal anchors depending on the particular item. For example, an item from the HADS-A subscale was "I feel tense or wound up: (1) most of the time, (2) a lot of the time, (3) from time to time, or (4) not at all." An item from the HADS-D subscale was "I feel as though I am slowed down: (1) nearly all of the time, (2) very often, (3) sometimes, or (4) not at all." The seven items from each respective subscale were summed to form an anxiety score and a depression score. Scores falling at or above eight on either the Depression or Anxiety subscales are considered to be clinically significant (Bjelland, Dahl, Haug, \& Neckelmann, 2002). High internal consistency (Cronbach's alpha $>0.8$ ) has been demonstrated in numerous studies examining individuals with cancer (Carroll, Kathol, Noyes Jr., Wald, \& Clamon, 1993; Moorey et al., 1991; Watson et al., 1999) and the internal reliabilities were similar within the present study for both the Anxiety (Cronbach's alpha $=.84)$ and Depression $($ Cronbach's alpha $=.82)$ scales.

Coping with Health Injuries and Problems Scale. The Coping with Health Injuries and Problems Scale (CHIP; Endler, Parker, \& Summerfeldt, 1998) is a validated self-report measure, which assesses coping strategies used by individuals experiencing physical health problems. Participants were asked to report their most recent illness or injury and then rate the extent to which they engaged in a variety of coping strategies when dealing with this illness/injury on a 5 -point Likert-type scale $(1=$ not at all; $5=$ 
very much). The measure contains 32 items which are divided into four subscales of health-specific coping dimensions: (1) Distraction (e.g., "Daydream") (2) Palliative (e.g., "Stay in bed"), (3) Instrumental (e.g., "Find out more information"), and (4) Emotional Preoccupation (e.g., "Feel angry"). The Distraction and Emotional Preoccupation subscales are considered emotion focused coping strategies, whereas the Palliative and Instrumental subscales are considered problem focused coping strategies. The CHIP has demonstrated internal reliability coefficients ranging from .75 to .83 in a sample of individuals experiencing a variety of medical conditions (Endler et al., 1998). Similarly, within the present study, internal reliability coefficients ranged from .74 (Distraction subscale) to .80 (Palliative subscale).

Worry Interference Scale. The Worry Interference Scale (WIS; Trask et al., 2001) is a self-report measure assessing the degree to which thoughts about breast cancer are perceived as interfering with one's daily functioning. For the purpose of the present study, the scale was adapted to assess the interference caused by Lynch syndrome as opposed to breast cancer. The WIS is composed of seven items, which are assessed on a 5-point multiple-choice scale ranging from "not at all" to "a lot." No clinical cutoffs are provided for the WIS, however, the authors state that any items that individuals report as interfering with their daily functioning should be considered as clinically relevant (Trask et al., 2001). Examples of items include; "Concerns about Lynch syndrome or cancer have affected my ability to have fun," and "Worries about Lynch syndrome have affected my ability to meet the needs of my family." The original WIS reported in the authors' validation paper included four additional items, which were subsequently removed due to pairwise correlations below .30. These items assessed participants' ability to speak with 
their partners about their concerns, their partners' abilities to understand their concerns, the degree to which cancer risk affected their decision to have children, and the frequency that participants worry about their children or grandchildren's chances of developing cancer. For the purpose of the present study, these four items were included for descriptive purposes; however, the original seven-item scale was used for quantitative analyses. The seven-item Worry Interference Scale has demonstrated excellent internal consistency in a sample of women attending a breast and ovarian cancer risk evaluation appointment, with Cronbach's alpha coefficients ranging from .89 to .94 (Trask et al., 2001). Within the present study, the internal consistency was also good (Cronbach's alpha $=.74)$.

\section{Statistical Analyses}

Power Analysis. The present study proposed utilizing a multiple regression analysis with six covariates (gender, age, time since diagnosis, age of children, previous diagnosis of cancer, and first degree relatives with cancer) and seven main predictors (palliative coping, instrumental coping, distraction based coping, emotional preoccupation coping, depression, anxiety, and worry interference). Using the statistical software G*Power 3 (Faul, Erdfelder, Lang, \& Buchner, 2007) an a priori power analyses indicated that it would be necessary to have analyzable data from at least 101 subjects to have $95 \%$ power for detecting a medium sized effect when employing the traditional .05 criterion of statistical significance.

Preliminary Analyses. Data were first screened to ensure that no errors were present. A frequency analysis was then run to examine missing data and to determine if there appeared to be any suspicious data. The data were also explored to ensure that they 
were normally distributed with no outliers. Furthermore, descriptive statistics regarding the patient demographic and health information was examined.

Given the possibility that some of the predictor variables would be highly interrelated, bivariate correlations between the predictors were also examined. If any predictor variables had a correlation of greater than .70 , the variable would be excluded to minimize multicollinearity in the model so as to not violate the statistical assumptions of linear regression analysis.

Hypothesis 1a. To describe the severity of health anxiety in a sample of individuals with Lynch syndrome, descriptive statistics illustrating the mean, median and range of health anxiety scores were conducted.

Hypothesis 2a. To examine whether demographic and medical variables predicted elevated health anxiety among patients diagnosed with Lynch Syndrome, a multiple linear regression analysis was conducted. In this analysis, health anxiety was the outcome variable and the variables of gender, age, time since diagnosis of either Lynch syndrome or first cancer, having a child under age 18, having a previous cancer diagnosis, and the number of first degree relatives with a cancer diagnosis were the predictor variables.

Hypothesis 2b. To examine whether the psychological and behavioural variables predicted elevated health anxiety among patients diagnosed with Lynch Syndrome, a multiple linear regression analysis was conducted. After controlling for the significant demographic and medical variables from hypothesis $2 a$ (i.e., gender, age, time since diagnosis of either Lynch syndrome or first cancer, having a child under age 18, having a previous cancer diagnosis, and the number of first degree relatives with a cancer 
diagnosis), the variables of palliative coping, instrumental coping, distraction based coping, emotional preoccupation coping, depression, anxiety, and worry interference were entered into the regression analysis. Health anxiety was the outcome variable.

Hypothesis 3. To examine the extent to which health anxiety predicted increased medical utilization by patients diagnosed with Lynch syndrome, a multiple linear regression analysis was conducted. In this analysis, gender, age, and history of cancer diagnosis were entered into the equation as covariates. Health anxiety was the predictor variable and frequency of medical utilization was the outcome variable. Medical utilization was examined as a total composite of all medical visits and was also examined separately based on the specific type of medical visit.

\section{Results}

\section{Preliminary Analyses}

All questionnaire data were entered and checked by the study primary investigator and research assistants. As a beginning step, data were screened to ensure that no errors were present. Specifically, a frequency analysis was conducted to examine missing data and to determine if there appeared to be any suspicious data points. Outliers were identified by standardized values of scale and subscale scores exceeding \pm 3.29 ( $p<.001$, 2-tailed). Results indicated that the SHAI had three outliers, the HADS-Anxiety had one outlier, the HADS-Depression had two outliers, and the WIS had two outliers. As recommended by Tabachnick and Fidell (2007), these outlying raw scores were changed to be one unit larger than the next most extreme non-outlying raw score in the distribution. However, altering the data in this manner did not impact any of the results and, as such, the data were returned to their original values. 
To assess the normality of the distributions, skewness and kurtosis of each of the key measures and subscales were assessed. Skewness and kurtosis were deemed to be minimal if the respective $\mathrm{z}$-scores fell between $\pm 2.58(p<0.01)$. Analyses revealed nonnormal distributions for a number of the study measures, including the SHAI, the HADSAnxiety, the HADS-Depression, and the WIS. Logarithm, square root, and reciprocal transformations were performed on these measures in an attempt to normalize the distributions; however, the transformations did not demonstrate a significant benefit in this regard. As such, the non-transformed data were used in all subsequent analyses.

Bivariate correlations between the key variables were assessed to identify any potential problems with multicollinearity. Examination of the correlation matrix (see Table 1) indicated that a number of the constructs were significantly related to one another. Specifically, for the main outcome variable of health anxiety, the SHAI was found to be weakly correlated with the CHIP-Palliative subscale and moderately correlated with the CHIP-Emotional Preoccupation subscale, the HADS-Anxiety, the HADS-Depression, and the WIS. Correlations between predictors ranged from .01 to .61. Importantly, none of the predictor variables had a correlation of greater than .70 and, therefore, did not violate the statistical assumptions around collinearity for linear regression analysis (Tabachnick \& Fidell, 2007).

\section{Sample Characteristics}

Demographics. In total, 209 individuals with a confirmed diagnosis of Lynch syndrome completed questionnaires for Study I. Table 2 displays the demographic characteristics of the sample. The majority of the sample was female (61.2\%), white $(90.9 \%)$, married or 
Table 1

Correlation Matrix of Key Psychological Variables

\begin{tabular}{|c|c|c|c|c|c|c|c|c|}
\hline & SHAI & CHIP-P & CHIP-I & CHIP-D & CHIP-E & HADS-A & HADS-D & WIS \\
\hline$\overline{\text { SHAI }}$ & 1.00 & $.15^{*}$ & -0.05 & 0.01 & $.50 * *$ & $.52 * *$ & $.45 * *$ & $.45^{* *}$ \\
\hline CHIP-P & & 1.00 & $.28 * *$ & $.27 * *$ & $.33 * *$ & 0.09 & 0.03 & 0.01 \\
\hline CHIP-I & & & 1.00 & $.61 * *$ & 0.14 & -0.11 & $-.25 * *$ & -0.12 \\
\hline CHIP-D & & & & 1.00 & $.28 * *$ & 0.01 & $-.16^{*}$ & -0.05 \\
\hline CHIP-E & & & & & 1.00 & $.39 * *$ & $.25 * *$ & $.36 * *$ \\
\hline HADS-A & & & & & & 1.00 & $.57 * *$ & $.33 * *$ \\
\hline HADS-D & & & & & & & 1.00 & $.37 * *$ \\
\hline WIS & & & & & & & & 1.00 \\
\hline
\end{tabular}

Note. SHAI=Health Anxiety Inventory (short version); CHIP-P=Palliative Coping Subscale; CHIP-I=Instrumental Coping Subscale; CHIP-D=Distraction Coping Subscale; CHIP-E=Emotional Preoccupation Coping Subscale; HADS-A=HADS Anxiety; HADS$\mathrm{D}=$ HADS Depression; WIS=Worry Interference Scale.

$*_{p}<.05, * * p<.01$. 
partnered (80.9\%; mean length of relationship $=25.47$ years, $S D=15.05$ years), employed (59.8\%), and college educated or higher (54.1\%). The age of participants ranged from 20 to 87 years old with a mean age of 53.22 years $(S D=14.53)$.

Children. The vast majority of study participants $(n=162,77.5 \%)$ had at least one biological child, with an average of 2.22 children $(S D=0.97$, range $1-6)$. A total of 51 individuals (31.5\%) had at least one child who was under the age of 18 years. In total, study participants provided information for 360 children, with 265 of the children over the age of 18. Examining only the children who were over 18 years of age (the current age guideline for predictive genetic testing of cancer susceptibility), 54.3\% $(n=144)$ had undergone genetic testing for Lynch syndrome. Of the children who had received genetic testing, $56.9 \%(n=82)$ tested positive as Lynch syndrome carriers.

Prior cancer diagnoses. A large proportion of the sample $(n=129,61.7 \%)$ had been diagnosed with at least one form of cancer over the course of their lives. The mean age that these individuals were diagnosed with their first cancer was 43.77 years $(S D=$ 10.29) and ranged between age 20 and 71. Among those diagnosed with cancer, the number of individual cancer diagnoses per patient (excluding non-melanoma skin cancers) ranged between one and five, with an average of $1.85(S D=1.17)$ cancer diagnoses per person. Recurrences of the same form of cancer were reported by $10.5 \%$ of the sample $(n=22)$. Colorectal cancer was the most commonly reported cancer $(n=$ $122,58.4 \%)$, followed by endometrial $(n=48,23.0 \%)$ and breast $(n=15,7.2 \%)$. A summary of patient medical characteristics can be seen in Table 3 and information related to the type of cancer, frequencies of diagnoses, and the number of cancers diagnosed can be seen in Table 4. 
Table 2

Study I Sample Demographics $(N=209)$

\begin{tabular}{lcc}
\hline Variable & $n(\%)$ & $M(S D)$ \\
\hline Age (years) & \\
Gender & $81(38.8)$ \\
$\quad$ Male & $128(61.2)$ \\
Female & \\
Ethnicity & $190(90.9)$ \\
White & $12(5.7)$ \\
Asian & $2(1.0)$ \\
Black & $1(0.5)$ \\
Aboriginal & $3(1.4)$ \\
Other & $59(28.2)$ \\
Employment status & $68(32.5)$ \\
Employed Full-time & \\
Employed Part-time & $59(28.2)$ \\
Retired & \\
Disability & \\
\hline Unemployed & \\
\hline
\end{tabular}


Table 2 (continued)

Study I Sample Demographics (N=209)

\begin{tabular}{lcc}
\hline Variable & $n(\%)$ & $M(S D)$ \\
\hline Education & $46(22.0)$ \\
High school or below & $45(21.5)$ \\
Some college & $83(39.7)$ \\
College & $30(14.4)$ \\
Graduate/professional & \\
Relationship Status & $169(80.9)$ \\
Married/partnered & $19(9.1)$ \\
Single & $9(4.3)$ \\
Widowed & $10(4.8)$ \\
Divorced/Separated & $1(0.5)$ \\
Other & \\
Biological Children & $162(77.5)$ \\
Yes & $47(22.5)$ \\
No &
\end{tabular}


Time since diagnosis of cancer or Lynch syndrome. Among the patients who had been diagnosed with cancer, the average duration of time since their first cancer diagnosis was 15.20 years $(S D=10.18)$, with a range between 0 and 41 years. Participants were not asked to provide their date of diagnosis of Lynch syndrome because this information was to be obtained from the FGICR database. Unfortunately, this information was only available for the patients who were diagnosed with Lynch syndrome at Mount Sinai Hospital specifically $(n=103)$ and not for the remaining patients diagnosed at other medical institutions. Among the individuals diagnosed at Mount Sinai Hospital, the average time since Lynch syndrome diagnosis was 5.43 years $(S D=4.05)$, with a range between 0.07 and 15.51 years. For the current research, the length of time an individual had been aware of their susceptibility to cancer was conceptualized as either the length of time since they were diagnosed with cancer or with Lynch syndrome, whichever came first. This information was available for 166 individuals. The average duration of time since participants were aware of their susceptibility to cancer (via cancer diagnosis or Lynch syndrome diagnosis) was 12.89 years $(S D=9.77)$, with a range between 0 and 41 years. The aforementioned data are presented in Table 3 .

Family history of cancer and Lynch syndrome. The FGICR database contained information related to family history of Lynch syndrome and family history of cancer for $194(92.8 \%)$ and 200 participants (95.7\%), respectively. The mean number of first degree family members with a confirmed diagnosis of Lynch syndrome was 1.46 $(S D=1.25$, range $0-5)$. Participants reported a significant family history of cancer. Specifically, the mean number of first degree relatives diagnosed with cancer was 2.21 
Table 3

Study I Medical Characteristics $(N=209)$

\begin{tabular}{lccc}
\hline Variable & $n(\%)$ & $M(S D)$ & Range \\
\hline History of cancer diagnosis & $129(61.7)$ & & \\
Age at first cancer diagnosis (years) & $43.77(10.29)$ & $20-71$ \\
$\begin{array}{l}\text { Total number of cancer diagnoses } \\
\text { Recurrence of same form of cancer }\end{array}$ & $1.85(1.17)$ & $1-5$ \\
$\begin{array}{l}\text { Time since first cancer diagnosis (years; } \\
n=129)\end{array}$ & $15.20(10.18)$ & $0-41$ \\
$\begin{array}{l}\text { Time since diagnosed with Lynch } \\
\text { syndrome (years; } n=103)\end{array}$ & $5.43(4.05)$ & $0.07-15.51$ \\
$\begin{array}{l}\text { Time since awareness of cancer } \\
\text { susceptibility (years; } n=166)\end{array}$ & $12.89(9.77)$ & $0-41$ \\
\hline
\end{tabular}


Table 4

Cancer Diagnoses $(N=209)$

\begin{tabular}{|c|c|c|c|c|c|c|}
\hline Type of Cancer & $\begin{array}{c}1^{\text {st }} \text { Cancer } \\
\mathrm{n}(\%)\end{array}$ & $\begin{array}{c}2^{\text {nd }} \text { Cancer } \\
n(\%)\end{array}$ & $\begin{array}{c}3^{\text {rd }} \text { Cancer } \\
\mathrm{n}(\%)\end{array}$ & $\begin{array}{c}4^{\text {th }} \text { Cancer } \\
\mathrm{n}(\%)\end{array}$ & $\begin{array}{c}5^{\text {th }} \text { Cancer } \\
\mathrm{n}(\%)\end{array}$ & $\begin{array}{c}\text { TOTAL } \\
\mathrm{n}(\%)\end{array}$ \\
\hline Colorectal & $86(41.14)$ & $21(10.04)$ & $10(4.78)$ & $2(0.96)$ & $4(1.91)$ & $122(58.37)$ \\
\hline Endometrial & $26(12.44)$ & $17(8.13)$ & $4(1.91)$ & $1(0.48)$ & 0 & $48(22.96)$ \\
\hline Breast & $4(1.91)$ & $4(1.91)$ & $4(1.91)$ & 0 & $3(1.43)$ & $15(7.17)$ \\
\hline Ovarian & $3(1.43)$ & $4(1.91)$ & $2(0.96)$ & 0 & $1(0.48)$ & $10(4.78)$ \\
\hline Prostate & 0 & $5(2.39)$ & $1(0.48)$ & $1(0.48)$ & 0 & $7(3.34)$ \\
\hline Renal & $1(0.48)$ & $2(0.96)$ & $2(0.96)$ & $1(0.48)$ & 0 & $6(2.87)$ \\
\hline Cervical & $4(1.91)$ & 0 & $1(0.48)$ & 0 & 0 & $5(2.39)$ \\
\hline Bladder & $1(0.48)$ & $1(0.48)$ & $1(0.48)$ & $2(0.96)$ & 0 & $5(2.39)$ \\
\hline Ureter & $1(0.48)$ & $2(0.96)$ & 0 & $1(0.48)$ & 0 & $4(1.91)$ \\
\hline Sarcoma & 0 & $2(0.96)$ & 0 & $2(0.96)$ & 0 & $4(1.91)$ \\
\hline Thyroid & $1(0.48)$ & 0 & $1(0.48)$ & 0 & 0 & $2(0.96)$ \\
\hline Duodenum & 0 & $1(0.48)$ & 0 & $1(0.48)$ & 0 & $2(0.96)$ \\
\hline Multiple Myeloma & $1(0.48)$ & 0 & 0 & 0 & 0 & $1(0.48)$ \\
\hline Non-Hodgkin Lymphoma & $1(0.48)$ & 0 & 0 & 0 & 0 & $1(0.48)$ \\
\hline
\end{tabular}


Table 4 (continued)

Cancer Diagnoses $(N=209)$

\begin{tabular}{lcccccc}
\hline & $\begin{array}{c}1^{\text {st }} \text { Cancer } \\
\mathrm{n}(\%)\end{array}$ & $\begin{array}{c}2^{\text {nd }} \text { Cancer } \\
\mathrm{n}(\%)\end{array}$ & $\begin{array}{c}3^{\text {rd }} \text { Cancer } \\
\mathrm{n}(\%)\end{array}$ & $\begin{array}{c}4^{\text {th }} \text { Cancer } \\
\mathrm{n}(\%)\end{array}$ & $\begin{array}{c}5^{\text {th }} \text { Cancer } \\
\mathrm{n}(\%)\end{array}$ & $\begin{array}{c}\text { TOTAL } \\
\mathrm{n}(\%)\end{array}$ \\
\hline Type of Cancer & 0 & $1(0.48)$ & 0 & 0 & 0 & $1(0.48)$ \\
Laryngeal & 0 & $1(0.48)$ & 0 & 0 & 0 & $1(0.48)$ \\
Liver & 0 & 0 & $1(0.48)$ & 0 & 0 & $1(0.48)$ \\
Stomach & 0 & 0 & $1(0.48)$ & 0 & 0 & $1(0.48)$ \\
Fallopian Tube & 0 & 0 & 0 & $1(0.48)$ & 0 & $1(0.48)$ \\
Melanoma & 0 & 0 & 0 & $1(0.48)$ & 0 & $1(0.48)$ \\
Spine & 0 & 0 & 0 & $1(0.48)$ & 0 & $1(0.48)$ \\
TOTAL & $128(61.24)$ & $61(29.18)$ & $28(13.39)$ & $14(6.70)$ & $8(3.83)$ & \\
\hline
\end{tabular}


$(S D=1.41)$, with a range between 0 and 7 family members. Data regarding the cause of family members' deaths were available for only 79 participants and demonstrated that a mean of 1.25 first-degree relatives $(S D=0.88$, range $0-3)$ were deceased from cancer.

\section{Descriptive Information on Key Variables}

Descriptive information is provided for key variables and subscales used in the present study. Table 5 provides the means, standard deviations, possible ranges, and reported ranges for the composite and subscales of the SHAI, the HADS-Depression and HADS-Anxiety Subscales, the subscales of the CHIP, and the total score of the WIS. Findings are outlined below.

Health anxiety. The mean total score on the SHAI was $13.68(S D=6.98)$. As previously noted, the SHAI contains two subscales: 1) Illness Likelihood, and 2) Negative Consequences. The Illness Likelihood subscale assesses the degree to which individuals are worried or preoccupied by a variety of bodily symptoms that are characteristic of severe health anxiety. Study participants' mean score on the 14-item Illness Likelihood subscale was $11.61(S D=5.95)$. The Negative Consequences subscale compliments the Illness Likelihood subscale by assessing the degree to which participants perceive the negative consequences of being ill. The mean score on the 4item Negative Consequences Subscale was $2.05(S D=1.85)$. A more thorough discussion of the SHAI scores is presented within the Hypothesis 1 results section.

Anxiety and depression. Overall, the mean scores on the Anxiety ( $M=5.46, S D$ $=3.84)$ and Depression $(M=2.75, S D=2.87)$ scales of the Hospital Anxiety and Depression Scale (HADS) were below the suggested clinical cut-offs of eight (Bjelland, Dahl, Haug, \& Neckelmann, 2002). However, over one quarter of the participants ( $n=$ 
Table 5

Means, Standard Deviations, and Ranges for Key Variables

\begin{tabular}{lcccc}
\hline & Cronbach's & & & \\
Key variable & Alpha & $M$ & Possible & Actual \\
Range & Range \\
\hline
\end{tabular}

Short Health Anxiety

Inventory (SHAI)

\begin{tabular}{|c|c|c|c|c|c|}
\hline Illness Likelihood & .87 & 11.61 & 5.95 & $0-42$ & $0.00-38.00$ \\
\hline Negative & & & & & \\
\hline Consequences & .67 & 2.05 & 1.85 & $0-12$ & $0.00-9.00$ \\
\hline Total Score & .87 & 13.68 & 6.98 & $0-54$ & $0.00-46.00$ \\
\hline
\end{tabular}

Hospital Anxiety and

Depression Scale

(HADS)

$\begin{array}{llllll}\text { Anxiety } & .84 & 5.46 & 3.84 & 0-21 & 0.00-19.00 \\ \text { Depression } & .82 & 2.75 & 2.87 & 0-21 & 0.00-16.00\end{array}$

Coping with Health

Injuries and Problems

Scale (CHIP)

$\begin{array}{lccccc}\text { Distraction } & .74 & 24.66 & 5.46 & 8-40 & 10.00-40.00 \\ \text { Palliative } & .80 & 25.52 & 5.39 & 8-40 & 8.00-40.00 \\ \text { Instrumental } & .77 & 31.06 & 4.94 & 8-40 & 15.00-40.00 \\ \begin{array}{l}\text { Emotional } \\ \text { Preoccupation }\end{array} & .78 & 20.87 & 5.71 & 8-40 & 8.00-35.00\end{array}$

Worry Interference

Scale (WIS)

74

$9.60 \quad 4.19 \quad 7-35 \quad 7.00-33.00$ 
$57 ; 27.5 \%$ ) scored higher than this clinical cut-off on the HADS Anxiety Scale. In contrast, far fewer participants $(n=14 ; 6.7 \%)$ scored at or above the same clinical cutoff for the HADS Depression Scale, indicating that anxiety was more prevalent within the current sample.

Coping strategies. In examining the scores on the Coping with Health Injuries and Problems Scale (CHIP), when faced with a health problem, participants were most likely to engage in Instrumental Coping (i.e., problem solving based coping; $M=31.06$, $S D=4.94$ ), followed by Palliative Coping (i.e., soothing strategies aimed at reducing discomfort associated with the health problem; $M=25.52, S D=5.39$ ) and Distraction Coping (i.e., distraction or avoidance of the health problem; $M=24.66, S D=5.46$ ). Participants were least likely to use Emotional Preoccupation as a coping strategy (i.e., focusing on the negative emotional consequences of the health problem; $M=20.87, S D=$ 5.71).

Worry interference. The mean score on the Worry Interference Scale was 9.60 $(S D=4.19)$. In total, 102 participants $(48.8 \%)$ indicated that their worry about cancer had interfered with their lives at least "a little," and 64 participants $(30.6 \%)$ answered 'sometimes,' 'often,' or 'always' to at least one of the seven cancer worry interference questions.

As previously noted in the Measures section, the original WIS contained an additional four items that are not included in the validated scale. However, these items were retained for descriptive purposes as they are relevant to the current study. The majority of participants reported difficulty communicating and feeling understood by their partners, with $54.5 \%(n=114)$ feeling as though they are "not at all" or "a little" 
able to talk to their partner about their cancer concerns and $56.5 \%(n=118)$ reported that their partner was able to understand their concerns "not at all" or "a little." Additionally, the vast majority $(n=164 ; 78.5 \%)$ reported that cancer risks did not affect their decision to have children, with only $5.7 \%(n=12)$ stating that cancer risks impacted their childbearing decision making "quite a bit" or "a lot." Despite the relatively low impact Lynch syndrome appeared to have on childbearing decisions, worry about the health of offspring was quite high in the sample. Specifically, $43.5 \%(n=91)$ reported that they worried about their children or grandchildren's chances of developing cancer "quite a bit" or "a lot."

Medical utilization. In total, 179 participants (85.6\%) indicated that over the past six months they attended at least one medical appointment, with an average of 3.53 $(S D=3.3$, range $=0-16)$ medical visits per person. General practitioners were the most commonly utilized for medical visits (172 visits, $M=1.73, S D=1.43$, range $=0-10$ ), followed by gastrointestinal specialists (115 visits, $M=.62, S D=.77$, range $=0-3$ ), surgeons $(110$ visits, $M=.68, S D=1.06$, range $=0-5)$, oncologists $(102$ visits, $M=.64$, $S D=1.69$, range $=0-12)$, “other" specialists $(101$ visits, $M=.73, S D=1.29$, range $=0$ -8 ), emergency room (99 visits, $M=.39, S D=.83$, range $=0-4$ ), genetic counsellors (93 visits, $M=.16, S D=.40$, range $=0-2)$, cardiologists $(85$ visits, $M=.14, S D=.44$, range $=0-2)$, and gynecologists (27 visits, $M=1.00, S D=.83$, range $=0-4)$. A summary of medical utilization is presented in Table 6.

\section{Hypothesis Testing}

Severity of health anxiety among Lynch syndrome carriers. Hypothesis 1 predicted that individuals with Lynch syndrome would report a wide range of health 
anxiety levels, but that a proportion would experience levels at or above the clinical cutoff. As hypothesized, participants reported a wide range of scores on the SHAI. The mean total score on the SHAI was $13.68(S D=6.98)$ and scores ranged from 0 to 38 (compared to the total possible scale range of 0 to 42). As clinical cutoff scores have only been established and reported for the Illness Likelihood Subscale (and not the Negative Consequences Subscale) of the Health Anxiety Inventory, results from the two subscales are presented separately. The mean score on the Negative Consequences Subscale was $2.05(S D=1.85)$. The mean score on the Illness Likelihood Subscale was $11.61(S D=5.95)$. On average, participants in the present study scored below the suggested clinical cut-off to identify severe health anxiety (cutoff score of $\geq 18$ ) or high health anxiety (cutoff score of $\geq 15)$ on the Illness Likelihood Subscale of the SHAI $(M=$ $11.61, S D=5.95)$. However, $16.7 \%$ of the sample $(n=35)$ was at or above the cut-off point for severe health anxiety and $29.3 \%$ of participants $(n=61)$ reported levels indicative of high health anxiety. The frequencies of participants meeting criteria for severe and high health anxiety are displayed in Table 7.

Further analyses were conducted to explore whether individuals who had been previously diagnosed with cancer reported higher health anxiety scores than individuals who had never had a cancer diagnosis. Independent samples t-tests revealed that there were no differences on the means of the SHAI total scores between those who had been diagnosed with cancer $(M=13.49, S D=7.37)$ and individuals who had not $(M=13.86$, $S D=6.24), t(191)=.37, p=.72$. Similarly, no differences were evident on the Illness Likelihood Subscale of the SHAI between those who had previously had cancer $(M=$ 
Table 6

Medical Visits over the Past Six Months

\begin{tabular}{lcccc}
\hline Type of Medical Visit & Total Visits & $M$ & $S D$ & Range \\
\hline Total Medical Utilization & 904 & 3.53 & 3.30 & $0-16$ \\
General Practitioner & 172 & 1.73 & 1.43 & $0-10$ \\
Gastroenterologist & 115 & .62 & .77 & $0-3$ \\
Surgeon & 110 & .68 & 1.06 & $0-5$ \\
Oncologist & 102 & .64 & 1.69 & $0-12$ \\
Other & 101 & .73 & 1.29 & $0-8$ \\
Emergency Room & 99 & .39 & .83 & $0-4$ \\
Genetic Counsellor & 93 & .16 & .40 & $0-2$ \\
Cardiologist & 85 & .14 & .44 & $0-2$ \\
Gynecologist & 27 & 1.00 & .83 & $0-4$ \\
\hline
\end{tabular}


Table 7

Frequency of Severe and High Health Anxiety based on the Health Anxiety Inventory $(N=209)$

\begin{tabular}{lc} 
& $n(\%)$ \\
\hline Severe Health Anxiety (cut-off $\geq 18)$ & $35(16.7)$ \\
Non-Severe Health Anxiety (cut-off $<18)$ & $174(83.3)$ \\
High Health Anxiety (cut-off $\geq 15)$ & $61(29.3)$ \\
Low Health Anxiety (cut-off $<15)$ & $148(70.8)$ \\
\hline
\end{tabular}


$11.45, S D=6.14)$ and those who have not $(M=11.88, S D=5.65), t(204)=.52, p=.60$. Nor were there any differences on the Negative Consequences subscale of the SHAI between those who have had cancer $(M=2.03, S D=1.91)$ and those who have not $(M=$ $2.08, S D=1.77) t(200)=.17, p=.87$. Additionally the percentage of participants meeting criteria for severe health anxiety and high health anxiety did not differ by cancer history, $\chi^{2}(2, \mathrm{~N}=206)=3.04, p=.22$.

Predictors of health anxiety. Hypothesis 2 aimed to identify predictors of health anxiety among individuals with Lynch syndrome. A variety of demographic and medical predictors were initially examined. Subsequent analyses examined psychological predictors of health anxiety among this population.

Demographic and medical predictors of health anxiety. In Hypothesis $2 \mathrm{a}$, health anxiety was expected to be higher in patients who were female, younger, who had younger children (i.e., < 18 years old), who had more experience (either personally or a first degree relative) with cancer, and who had been more recently diagnosed with Lynch syndrome or cancer. The results from the multiple regression analysis are displayed in Table 8 . Results indicated that the overall model was statistically significant, $F(7,146)=$ $3.06, p=.005$, but only younger age was significantly associated with higher health anxiety, $\beta=-.25, p=.05$. Contrary to the hypothesis, gender, education, having younger children, being previously diagnosed with cancer, the number of first-degree relatives diagnosed with cancer, or being more recently diagnosed with Lynch syndrome or cancer all were not significantly related to health anxiety. The adjusted $R^{2}$ value of the model was .09 , indicating that $9 \%$ of the variance in health anxiety was accounted for by the model. 


\section{Table 8}

Hypothesis 2a: Multiple Regression Analysis of Demographic and Medical Predictors of Health Anxiety

\begin{tabular}{|c|c|c|c|}
\hline Variable & $\boldsymbol{B}$ & $S E(B)$ & $\beta$ \\
\hline Gender & -.14 & 1.06 & -.01 \\
\hline Age & -.12 & .06 & $-.25^{*}$ \\
\hline Education & -2.28 & .06 & -.15 \\
\hline $\begin{array}{l}\text { Time since diagnosis } \\
\text { (Lynch syndrome or } \\
\text { cancer) }\end{array}$ & -.04 & .07 & -.06 \\
\hline Children under 18 & 1.41 & 1.41 & .09 \\
\hline $\begin{array}{l}\text { Previous cancer } \\
\text { diagnosis }\end{array}$ & -.21 & 1.46 & -.01 \\
\hline Relatives with cancer & .01 & .43 & .01 \\
\hline
\end{tabular}

Note. Total $F(7,146)=3.06^{* *}$, Adjusted $R^{2}=.09$.

$* p<.05, * * p<.01, * * * p<.001$. 
Psychological predictors of health anxiety. In Hypothesis 2b, psychological variables were expected to have a significant additive effect in predicting health anxiety, above and beyond the variance accounted for by demographic and medical variables. Specifically, it was hypothesized that greater health anxiety would be predicted by more use of emotional preoccupation and distraction based coping strategies, less use of palliative and instrumental based coping strategies, greater depression and anxiety, and greater worry interference. As age was the only significant predictor of health anxiety in Hypothesis 2a, this was the only covariate entered in Block 1.

Table 9 displays the results of the hierarchical regression analysis. In Block 1, the overall model was significant, $F(1,187)=10.58, p=.001$, with younger age being associated with higher health anxiety, $\beta=-.23, p=.001$. The adjusted $\mathrm{R}^{2}$ for Block 1 was .05 , indicating that approximately $5 \%$ of the variance in health anxiety was accounted for by age. The overall model in Block 2 was significant, $F(8,180)=20.28, \mathrm{p}=.001$. Younger age, $\beta=-.12, p=.046$, increased use of the coping strategy of emotional preoccupation, $\beta$ $=.28, p=.001$, higher anxiety, $\beta=.25, \mathrm{p}=.001$, higher depression, $\beta=.18, \mathrm{p}=.02$, and greater worry interference, $\beta=.20, p=.002$, were all significantly associated with greater health anxiety. The $\mathrm{R}^{2}$ change was .42 and was statistically significant, $F(7,180)=$ $20.56, p=.001$, indicating that an additional $42 \%$ of the variance in health anxiety was accounted for by these psychological variables. Overall, the total adjusted $R^{2}$ for the final model accounted for $45.1 \%$ of the variance in health anxiety.

Consequences of health anxiety. Hypothesis 3 predicted that increased medical utilization would be predicted by greater health anxiety, above and beyond the variance accounted for by gender, age, and history of cancer diagnosis. Medical utilization was 
Table 9

Hypothesis 2b: Psychological Predictors of Health Anxiety

\begin{tabular}{llll}
\hline Variable & B & SE(B) & $\beta$ \\
\hline
\end{tabular}

Block 1
Age
$-.12$
.04
$-.23 * * *$

Block 2

$\begin{array}{lccc}\text { Age } & -.06 & .03 & -.12^{*} \\ \text { CHIP Distraction } & -.16 & .09 & -.12 \\ \text { CHIP Palliative } & 6.47 & .08 & .00 \\ \text { CHIP Instrumental } & .15 & .10 & .10 \\ \text { CHIP Emotional } & .35 & .09 & .28^{* * *} \\ \text { Preoccupation } & & & .25^{* * *} \\ \text { HADS Anxiety } & .47 & .13 & .18^{*} \\ \text { HADS Depression } & .44 & .18 & .20^{* *} \\ \text { WIS } & & .10 & \end{array}$

$\overline{\text { Note. Block 1: Total } \mathrm{F}(1,187)=10.58^{* * *}, \text { Adjusted } \mathrm{R}^{2}=.05 . \text { Block 2: Total } \mathrm{F}(8,180)}=$ $20.28 * * *$, Adjusted $\mathrm{R}^{2}=.45$.

$* \mathrm{p}<.05, * * \mathrm{p}<.01, * * * p<.001$. 
examined as a total composite of all medical visits and was also examined separately based on the specific type of medical visit. Table 10 displays the results of the hierarchical regression analyses.

Total medical utilization. In Block 1, the overall model was significant, $F(3,182)$ $=3.57, p=.02$, adjusted $\mathrm{R}^{2}=.04$. The only demographic or medical variable significantly associated with increased total medical utilization was having a previous history of a cancer diagnosis, $\beta=.21, p=.01$. The overall model in Block 2 was also significant, $F(4,181)=3.96, p=.004$. Higher health anxiety, $\beta=.16, p=.03$ was significantly associated with greater medical utilization. The $\mathrm{R}^{2}$ change was .03 and was statistically significant, $F(1,181)=.03, p=.03$, indicating that an additional $3.0 \%$ of the variance in medical utilization was accounted for by health anxiety. Overall, the total adjusted $\mathrm{R}^{2}$ for the final model accounted for $6.0 \%$ of the variance in total medical utilization.

General practitioner visits. In Block 1, the overall model was significant, $F(3$, 164) $=5.56, p=.001$, adjusted $\mathrm{R}^{2}=.08$. Only female gender was significantly associated with increased visits to a general practitioner, $\beta=.22, p=.004$. The overall model in Block 2 was also significant, $F(4,163)=4.23, p=.003$. However, health anxiety was not significantly associated with general practitioner visits, $\beta=.04, p=.58$. The $\mathrm{R}^{2}$ change was .002 and was not statistically significant, $F(1,163)=.30, p=.58$. Overall, the total adjusted $\mathrm{R}^{2}$ for the final model accounted for $7.2 \%$ of the variance in total general practitioner visits. 
Table 10

Hypothesis 3: Consequences of Health Anxiety

\begin{tabular}{|c|c|c|c|c|c|c|c|c|c|}
\hline \multirow[b]{2}{*}{ Variable } & \multicolumn{3}{|c|}{ Total Medical Utilization } & \multicolumn{3}{|c|}{ General Practitioner Visits } & \multicolumn{3}{|c|}{ Oncologist Visits } \\
\hline & $B$ & $S E(B)$ & $\beta$ & $B$ & $S E(B)$ & $\beta$ & $B$ & $S E(B)$ & $\beta$ \\
\hline \multicolumn{10}{|l|}{ Block 1} \\
\hline Gender & .70 & .49 & .10 & .62 & .21 & $.22 * *$ & .30 & .40 & .08 \\
\hline Age & -.01 & .02 & -.01 & .01 & .01 & .11 & -.01 & .01 & -.03 \\
\hline Previous Cancer Diagnosis & 1.44 & .57 & $.21 * *$ & .40 & .25 & .14 & .90 & .40 & $.25^{*}$ \\
\hline$F$ & & $3.57 *$ & & & $5.56 * * *$ & & & 2.38 & \\
\hline Adjusted $R^{2}$ & & .04 & & & .08 & & & .04 & \\
\hline \multicolumn{10}{|l|}{ Block 2} \\
\hline Gender & .73 & .48 & .11 & .63 & .22 & $.22 * *$ & .29 & .40 & .07 \\
\hline Age & .01 & .02 & .04 & .01 & .01 & .12 & -.01 & .01 & -.02 \\
\hline Previous Cancer Diagnosis & 1.36 & .56 & $.20^{*}$ & .39 & .25 & .13 & .88 & .40 & $.25^{*}$ \\
\hline SHAI Total Score & .08 & .03 & $.16^{*}$ & .01 & .02 & .04 & .01 & .02 & .05 \\
\hline$F$ & & $3.96^{* *}$ & & & $4.23 * *$ & & & 1.82 & \\
\hline Adjusted $R^{2}$ & & .06 & & & .07 & & & .03 & \\
\hline
\end{tabular}

Note: $* p<.05, * * p<.01, * * * p<.001$. 
Table 10 (continued)

Hypothesis 3: Consequences of Health Anxiety

\begin{tabular}{|c|c|c|c|c|c|c|c|c|c|}
\hline \multirow[b]{2}{*}{ Variable } & \multicolumn{3}{|c|}{ Surgeon Visits } & \multicolumn{3}{|c|}{ Gastroenterologist Visits } & \multicolumn{3}{|c|}{ Cardiologist Visits } \\
\hline & $B$ & $S E(B)$ & $\beta$ & $B$ & $S E(B)$ & $\beta$ & $B$ & $S E(B)$ & $\beta$ \\
\hline \multicolumn{10}{|l|}{ Block 1} \\
\hline Gender & 1.14 & .19 & $.50 * * *$ & .59 & .14 & $.36 * * *$ & .60 & .13 & $.45 * * *$ \\
\hline Age & -.01 & .01 & -.04 & .01 & .01 & .16 & .01 & .01 & $.32 * *$ \\
\hline Previous Cancer Diagnosis & .52 & .21 & $.23 *$ & .07 & .15 & .05 & -.04 & .10 & -.05 \\
\hline$F$ & \multicolumn{3}{|c|}{$15.74 * * *$} & \multicolumn{3}{|c|}{$6.58 * * *$} & \multicolumn{3}{|c|}{$8.67 * * *$} \\
\hline Adjusted $R^{2}$ & \multicolumn{3}{|c|}{.29} & \multicolumn{3}{|c|}{.13} & \multicolumn{3}{|c|}{.22} \\
\hline \multicolumn{10}{|l|}{ Block 2} \\
\hline Gender & 1.14 & .19 & $.49 * * *$ & .59 & .14 & $.36 * *$ & .60 & .13 & $.45 * * *$ \\
\hline Age & -.01 & .01 & -.04 & .01 & .01 & $.20^{*}$ & .01 & .01 & $.32 * *$ \\
\hline Previous Cancer Diagnosis & .52 & .21 & $.23 *$ & .05 & .15 & .03 & -.04 & .10 & -.05 \\
\hline SHAI Total Score & .01 & .01 & .01 & .02 & .01 & $.21 *$ & .01 & .01 & .02 \\
\hline$F$ & \multicolumn{3}{|c|}{$11.69 * * *$} & \multicolumn{3}{|c|}{$6.51 * * *$} & \multicolumn{3}{|c|}{$6.44 * * *$} \\
\hline Adjusted $R^{2}$ & \multicolumn{3}{|c|}{.29} & \multicolumn{3}{|c|}{.16} & \multicolumn{3}{|c|}{.21} \\
\hline
\end{tabular}

Note: $* p<.05, * * p<.01, * * * p<.001$. 
Table 10 (continued)

Hypothesis 3: Consequences of Health Anxiety

\begin{tabular}{|c|c|c|c|c|c|c|c|c|c|}
\hline \multirow[b]{2}{*}{ Variable } & \multicolumn{3}{|c|}{ Gynecologist Visits } & \multicolumn{3}{|c|}{ Genetic Counselling Visits } & \multicolumn{3}{|c|}{ Emergency Room Visits } \\
\hline & $B$ & $S E(B)$ & $\beta$ & $B$ & $S E(B)$ & $\beta$ & $B$ & $S E(B)$ & $\beta$ \\
\hline \multicolumn{10}{|l|}{ Block 1} \\
\hline Gender & -- & -- & -- & .59 & .09 & $.57 * * *$ & 1.18 & .17 & $.60 * * *$ \\
\hline Age & -.03 & .02 & -.41 & -.01 & .01 & -.04 & .01 & .01 & .14 \\
\hline Previous Cancer Diagnosis & .23 & .39 & .14 & .11 & .08 & .13 & .05 & .16 & .03 \\
\hline$F$ & & 1.48 & & & $15.57 * * *$ & & & $16.98 * * *$ & \\
\hline Adjusted $R^{2}$ & & .04 & & & .33 & & & .33 & \\
\hline \multicolumn{10}{|l|}{ Block 2} \\
\hline Gender & -- & -- & -- & .59 & .09 & $.57 * * *$ & 1.15 & .16 & $.59 * * *$ \\
\hline Age & -.03 & .02 & -.46 & -.01 & .01 & -.03 & .01 & .01 & .17 \\
\hline Previous Cancer Diagnosis & .27 & .41 & .17 & .11 & .08 & .13 & .02 & .16 & .01 \\
\hline SHAI Total Score & -.01 & .02 & -.11 & .01 & .01 & .07 & .02 & .01 & $.17^{*}$ \\
\hline$F$ & & 1.04 & & & $11.79 * * *$ & & & $14.25 * * *$ & \\
\hline Adjusted $R^{2}$ & & .01 & & & .35 & & & .36 & \\
\hline
\end{tabular}

Note: $* p<.05, * * p<.01, * * * p<.001$. 
Table 10 (continued)

Hypothesis 3: Consequences of Health Anxiety

\begin{tabular}{lccc}
\hline & \multicolumn{3}{c}{ Other Medical Visits } \\
\cline { 2 - 4 } Variable & $B$ & $S E(B)$ & $\beta$ \\
\hline Block 1 & 1.57 & .26 & $.54^{* * *}$ \\
Gender & -.01 & .01 & -.04 \\
Age & -.07 & .26 & -.03 \\
Previous Cancer Diagnosis & & $12.79 * * *$ & \\
F & & .26 & \\
Adjusted $R^{2}$ & & & \\
Block 2 & & & \\
Gender & 1.56 & .26 & $.53^{* * *}$ \\
Age & -.01 & .01 & -.03 \\
Previous Cancer Diagnosis & -.06 & .26 & -.02 \\
SHAI Total Score & .02 & .02 & .10 \\
$F$ & & $9.97 * * *$ & \\
Adjusted $R^{2}$ & & .26 & \\
& & &
\end{tabular}

Note: $* p<.05, * * p<.01, * * * p<.001$. 
Oncologist visits. In Block 1, the overall model was not significant, $F(3,96)=$ $2.38, p=.08$, adjusted $\mathrm{R}^{2}=.04$. The overall model in Block 2 was also not significant, $F(4,95)=1.82, p=.13$ and health anxiety was not associated with increased visits to the oncologist, $\beta=.05, p=.65$. The $\mathrm{R}^{2}$ change was .002 and was not statistically significant, $F(1,95)=.21, p=.65$. Overall, the total adjusted $\mathrm{R}^{2}$ for the final model accounted for $3.2 \%$ of the variance in total medical utilization.

Surgeon visits. In Block 1 , the overall model was significant, $F(3,103)=15.74, p$ $=.001$, adjusted $\mathrm{R}^{2}=.29$. Female gender, $\beta=.49, p=.001$, and having a personal history of a cancer diagnosis, $\beta=.23, p=.01$, were both associated with increased surgeon visits. The overall model in Block 2 was also significant, $F(4,102)=11.69, p=$ .001 . However, health anxiety was not significantly associated with surgeon visits, $\beta=$ $.001, p=.99$. The $\mathrm{R}^{2}$ change was .00 and was not statistically significant, $F(1,102)=$ $.00, p=.99$. Overall, the total adjusted $\mathrm{R}^{2}$ for the final model accounted for $2.9 \%$ of the variance in surgeon visits.

Gastroenterologist Visits. In Block 1, the overall model was significant, $F(3,109)$ $=6.58, p=.001$, adjusted $\mathrm{R}^{2}=.13$. Only female gender was associated with increased gastroenterologist visits, $\beta=.36, p=.001$. The overall model in Block 2 was also significant, $F(4,108)=6.51, p=.001$. Higher health anxiety was significantly associated with more gastroenterologist visits, $\beta=.21, p=.02$. The $\mathrm{R}^{2}$ change was .04 and was statistically significant, $F(1,108)=5.48, p=.02$, indicating that an additional $4 \%$ of the variance in gastroenterologist visits was accounted for by health anxiety. Overall, the total adjusted $\mathrm{R}^{2}$ for the final model accounted for $16.4 \%$ of the variance in total gastroenterologist visits. 
Cardiologist visits. In Block 1, the overall model was significant, $F(3,78)=8.67$, $p=.001$, adjusted $\mathrm{R}^{2}=.22$. Older age, $\beta=.32, p=.004$, and male gender, $\beta=.45, p=$ .001 , were significantly associated with increased cardiologist visits. The overall model in Block 2 was also significant, $F(4,77)=6.44, p=.001$. However, health anxiety was not significantly associated with cardiologist visits, $\beta=.02, p=.84$. The $\mathrm{R}^{2}$ change was .00 and was not statistically significant, $F(1,77)=.04, p=.84$. Overall, the total adjusted $\mathrm{R}^{2}$ for the final model accounted for $21.2 \%$ of the variance in cardiologist visits.

Gynecologist visits. Gender was not entered into Block 1 of this regression due to the fact that only female patients are seen by gynecologists. In Block 1, the overall model was not significant, $F(2,22)=1.48, p=.25$, adjusted $\mathrm{R}^{2}=.04$. No demographic or medical variables were significantly associated with increased gynecologist visits. The overall model in Block 2 was also not significant, $F(3,21)=1.04, p=.39$ and health anxiety was not associated with increased visits to the gynecologist, $\beta=-.11, p=.61$. The $\mathrm{R}^{2}$ change was .01 and was not statistically significant, $F(1,21)=.26, p=.61$. Overall, the total adjusted $\mathrm{R}^{2}$ for the final model accounted for $.5 \%$ of the variance in total gynecologist visits.

Genetic counselling visits. In Block 1, the overall model was significant, $F(3$, 87) $=15.57, p=.001$, adjusted $\mathrm{R}^{2}=.33$. Female gender was significantly associated with more genetic counselling visits, $\beta=.57, p=.001$. The overall model in Block 2 was also significant, $F(4,86)=11.79, p=.001$. However, health anxiety was not significantly associated with genetic counselling visits, $\beta=.07, p=.42$. The $\mathrm{R}^{2}$ change was .01 and was not statistically significant, $F(1,86)=.65, p=.42$. Overall, the total 
adjusted $\mathrm{R}^{2}$ for the final model accounted for $32.4 \%$ of the variance in genetic counselling visits.

Emergency room visits. In Block 1, the overall model was significant, $F(3,93)=$ $7.94, p=.001$, adjusted $\mathrm{R}^{2}=.33$. Female gender was significantly associated with increased emergency room visits, $\beta=.59, p=.001$. The overall model in Block 2 was also significant, $F(4,92)=14.25, p=.001$. Higher health anxiety, $\beta=.17, p=.04$ was significantly associated with more emergency room visits. The $\mathrm{R}^{2}$ change was .03 and was statistically significant, $F(1,92)=4.25, p=.04$, indicating that an additional $3 \%$ of the variance in emergency room visits was accounted for by health anxiety. Overall, the total adjusted $\mathrm{R}^{2}$ for the final model accounted for $35.6 \%$ of the variance in total emergency room visits.

Other medical visits. In Block 1, the overall model was significant, $F(3,97)=$ $12.79, p=.001$, adjusted $\mathrm{R}^{2}=.26$. Female gender was significantly associated with increased visits to other medical professionals (e.g., psychologist, chiropractor, massage therapist, etc.), $\beta=.54, p=.001$. The overall model in Block 2 was also significant, $F(4$, $96)=9.97, p=.001$. However, health anxiety was not significantly associated with visits to other medical professionals, $\beta=.10, p=.25$. The $\mathrm{R}^{2}$ change was .01 and was not statistically significant, $F(1,96)=1.36, p=.25$. Overall, the total adjusted $\mathrm{R}^{2}$ for the final model accounted for $26.4 \%$ of the variance in visits to other medical professionals.

\section{Discussion}

The purpose of the present study was to determine the extent to which health anxiety is a concern for individuals who have been diagnosed with Lynch syndrome. Additionally, the study was designed to examine demographic, medical, and 
psychological variables that predict elevated health anxiety and also investigate the impact of high health anxiety on medical utilization by Lynch syndrome patients.

\section{Current Findings}

\section{Severity of Health Anxiety Among Lynch Syndrome Carriers.}

Health anxiety among Lynch syndrome carriers. Consistent with Hypothesis 1, the degree of health anxiety experienced by Lynch syndrome carriers varied, ranging from absent to severe. However, approximately $30 \%$ of the sample reported clinically significant levels of health anxiety and, within that group, $17 \%$ of the total sample fell within the range expected among individuals meeting criteria for severe health anxiety. This prevalence rate becomes particularly notable when compared to the estimated three to six percent of the general (i.e., healthy) population that experiences high health anxiety (Bleichhardt \& Hiller, 2007; Sunderland et al., 2012), highlighting that health anxiety is a significant concern for individuals with Lynch syndrome.

Percentage of Lynch syndrome carriers meeting criteria for high health anxiety versus other populations. The finding that just under one-third of the sample of Lynch syndrome carriers reported experiencing significant health anxiety or symptoms consistent with a severe level of health anxiety is consistent with other published medical samples. Specifically, the proportion of Lynch syndrome carriers reporting severe health anxiety was just slightly less than what has been reported among patients with current cancer diagnoses. For example, Stark et al. (2004), in a sample of patients with breast or testicular cancers, found that $33 \%$ reported high health anxiety and Grassi and colleagues (2004) found that $38 \%$ of women with breast cancer experienced significant health anxiety. Moreover, the rate of high health anxiety among our sample was higher than 
that reported in other non-cancer afflicted medical populations. Specifically, only $16 \%$ of patients awaiting a surgical procedure (Janzen \& Hadjistavropoulos, 2008) and 24.9\% of patients with multiple sclerosis (Kehler \& Hadjistavropoulos, 2009) reported high levels of health anxiety.

It was unexpected that the percentage of individuals meeting criteria for high health anxiety was higher in our sample compared to other medical populations (e.g., patients awaiting surgery, individuals with multiple sclerosis; Janzen \& Hadjistavropoulos, 2008; Kehler \& Hadjistavropoulos, 2009). One explanation for this may be as simple as the hallmark differences between the health conditions. For example, patients awaiting surgery may experience procedure-related anxiety, however, they are presumably not facing a chronic condition. Moreover, the course of multiple sclerosis is relapsing and remitting, does not have a familial component, and typically does not pose an immediate threat of death. Additionally, given that individuals with Lynch syndrome are aware that they have up to an $85 \%$ chance of developing cancer (Jenkins et al., 2006) and that cancer will likely occur at a younger age and progress more rapidly than sporadic cancers (Lindor et al., 2006; Mitchell et al., 2002) it is reasonable to assume that this awareness may lead to an increase in health anxiety.

\section{Average health anxiety scores for Lynch syndrome carriers versus other}

populations. The mean scores on the SHAI from the Lynch syndrome carriers in our study were also compared to published scores obtained from other medical and non- 
medical populations ${ }^{2}$. Specifically, health anxiety among Lynch syndrome carriers was compared to a sample of patients attending a gastroenterologist clinic (Salvoskis et al., 2002). Although the specific reason for attending the gastroenterologist clinic was not reported, it can be assumed that this patient group was comprised of a combination of individuals who had been diagnosed with digestive conditions, diseases of the liver or pancreas, or who were receiving testing or screening procedures (e.g., colonoscopy), as these are the most common reasons for seeing a gastroenterologist (American Gastroenterological Association, 2013). This, in combination with the fact that Lynch syndrome patients typically see gastroenterologists a minimum of once per year and the similarities in demographics (mean age $=47$ years; $61.2 \%$ females), allow for this sample to be a reasonable medical comparison group to the Lynch syndrome population. The overall health anxiety of our sample $(M=13.68, S D=6.98)$ was comparable to the health anxiety in the gastroenterologist clinic group $(M=13.9, S D=7.4)$. Similarly, no significant differences were observed on the Illness Likelihood subscale (i.e., a subscale designed to assess emotions and attitudes related to acquiring a serious medical condition; e.g., "I spend most of my time worrying about my health") between the two groups. Surprisingly, the mean scores on the Negative Consequences subscale (i.e., a subscale that assesses the feared burden of acquiring a serious illness; e.g., "A serious illness would ruin every aspect of my life") were significantly lower in our sample than in gastrointestinal patients.

\footnotetext{
${ }^{2}$ Independent samples t-tests were conducted post hoc to compare the mean health anxiety scores on the SHAI to various populations reported in Salkovskis and colleagues' (2002) SHAI scale validation paper.
} 
Moreover, health anxiety among Lynch syndrome carriers appears to be higher than what is observed within the general population. The mean health anxiety total score as well as the Illness Likelihood subscale on the SHAI was higher for Lynch syndrome carriers than for a non-clinical, community based control group with similar demographics to the current sample (Salkovskis et al., 2002). Similar to the pattern found for patients attending a gastrointestinal clinic, the mean scores on the Negative Consequences subscale were significantly lower in our patients compared to the nonclinical control group.

Although several of our findings are consistent with prior literature, one inconsistency is that Lynch syndrome patients scored significantly lower on the Negative Consequences subscale of the SHAI than both the gastrointestinal patients and the nonclinical control group. This finding suggests that individuals with Lynch syndrome have significantly less fears about the burden of acquiring a serious medical illness, which may be explained by contextual factors. One possibility is that because individuals with Lynch syndrome frequently undergo cancer-screening procedures, they may feel confident that any cancers would be caught early and, thus, treated effectively. It is important to note that these beliefs are not unfounded, particularly with regards to colorectal cancer. Regular colonoscopies, for example, can prevent colon cancer by identifying and removing polyps before they become cancer. Additionally, if colorectal cancer is detected and treated in the early stages, the survival rate is an impressive $90 \%$ (American Cancer Society, 2011). Further support for this explanation comes from patients who have undergone genetic testing for hereditary cancers and also echo these beliefs. For example, in two separate studies, the vast majority of individuals who tested 
positive for hereditary breast and ovarian cancer stated that the primary benefits of knowing their mutation carrier status was increased access to screening procedures and surgical options (Claes, Evers-Kiebooms, Denayer, Decruyenaere, Moogaerts, Philippe, \& Legius, 2005; Lim et al., 2004). Similarly, among Lynch syndrome carriers, a substantial proportion report feeling optimistic about their futures because of the prevention and early detection methods available (Palmquist, Koehly, Peterson, Shegog, Vernon, \& Gritz, 2010; Peterson, Esplen, Ladelund, Bernstein, Sunde, Carlsson, \& Nilbert, 2011). Additionally, our sample's lived experience and/or experiential knowledge with Lynch syndrome may account for their lower scores on the Negative Consequences subscale. Given that nearly everyone in our sample has either had cancer or had a family member diagnosed with cancer, the patients have already had to grapple with (either first-hand or vicariously) many of the feared burdens of being diagnosed with cancer. It is possible that based on their lived experiences, many of these individuals view cancer as treatable and, as such, the negative consequences are not perceived to be as extreme. Overall, it appears that for many individuals with Lynch syndrome, being aware of their cancer risk and the ability of modern medicine to appropriately manage their health and their first-hand experience with cancer may positively impact the feared consequences associated with developing cancer.

Relatedly, high rates of cancer in our sample may partially account for their higher health anxiety compared to healthy individuals, but analogous health anxiety compared to other published samples of cancer patients. Within our sample, over $60 \%$ of the sample has had cancer at least once in their lifetime, with $11.5 \%(n=24)$ of the sample diagnosed with a cancer within the past two years and 9.6\% $(\mathrm{n}=20)$ diagnosed 
within the past 12 months. One of the most commonly reported concerns among cancer patients is fear of recurrence. This construct, which is closely related to health anxiety, endures for many years after recovery (Koch, Jansen, Brenner, \& Arndt, 2013).

Therefore, it is possible that the conceptual overlap between health anxiety and fear of recurrence could partially explain high health anxiety in our sample. However, it is also important to note that no significant differences were observed on health anxiety between individuals who had been previously diagnosed with cancer and those who had not. It appears that, overall, there are more similarities than differences present between Lynch syndrome carriers who have never been diagnosed with cancer and individuals who have been previously diagnosed with cancer. From a psychological perspective, the fear of developing cancer for the first time and the fear of a cancer recurrence would be captured in similar ways on a measure of health anxiety. Moreover, both cancer survivors and individuals with Lynch syndrome are constantly being monitored for cancers (e.g., yearly colonoscopies). As such, the medical and psychological experiences of Lynch syndrome carriers appear to be quite similar to that of individuals diagnosed with cancer.

\section{Predictors of Health Anxiety.}

Demographic and medical predictors of health anxiety. Hypothesis 2 a aimed to identify demographic and medical predictors of health anxiety among Lynch syndrome carriers. Contrary to what was hypothesized, gender, level of education, having younger children, being previously diagnosed with cancer, the number of first degree relatives diagnosed with cancer, and being more recently diagnosed with Lynch syndrome or cancer did not significantly predict health anxiety among this population. In fact, the 
only variable that was significantly related to health anxiety was age, with younger patients experiencing higher levels of health anxiety than their older counterparts.

Although this is the first study to examine health anxiety as an outcome variable among individuals with Lynch syndrome, previous research has identified a number of demographic and medical variables associated with increased psychological distress (e.g., depression, anxiety, cancer-specific worries) among patients being tested for Lynch syndrome. Despite research in this area being somewhat limited, the following summary of variables has been shown to be linked to greater psychological distress among those with Lynch syndrome: being female, level of education, having a greater family history of cancer, having lost a parent to cancer, having younger children, having increased exposure to cancer screening procedures, and having a parent diagnosed with cancer during childhood (d'Agincourt-Canning, 2005; Collins, Halliday, Warren, \& Williamson, 2000; Esplen et al., 2003; Gritz et al., 2005; Keller et al., 2002, 2008; Mcallister, 2002; Meiser, 2005; Rimes et al., 2006; Van Oostrom et al., 2006; Vernon, Gritz, Peterson, Amos, Perz, Baile \& Lynch, 1997). Similarly, from the broader literature examining individuals diagnosed with cancer and those being tested for genetic susceptibility to cancer (e.g., HBOC), younger age, having a greater family history of cancer, losing a parent to cancer, and having younger children have been shown to be associated with increased anxiety about developing cancer (Jones et al., 2012; Rimes et al., 2006; Tilburt et al., 2011; Van Oostrom et al., 2006). Medical variables such as time since receiving a diagnosis of cancer, experiencing a previous major medical illness, and greater past exposure to prophylactic tests or treatments have also been linked to increased patient anxiety (Jones et al., 2012). 
It is unclear exactly why our demographic and medical predictors were not associated with increased health anxiety; however, there are several possibilities that warrant discussion. One possible explanation is that the broader variable of psychological distress is not an appropriate proxy on which to base our a priori hypothesized predictors of health anxiety. Without doubt, health anxiety certainly represents a portion of what constitutes the larger umbrella term of "psychological distress." For example, among medical populations, health anxiety has been shown to be related to distress, depression and general anxiety (Hadjistavropoulos et al., 2012). However, health anxiety is a more specific and refined concept that focuses on the heightened and persistent anxiety individuals experience due to perceived threats to their health. The assessment of beliefs about the likelihood of becoming ill, the severity of feared illnesses, or one's perceived ability to cope with an illness is quite different from general psychological distress questionnaires that may ask participants to report on their general mood or the physiological components of anxiety. Thus, factors that are predictive of the broader psychological distress may not directly translate to health anxiety. Our lack of findings is also supported by population-based studies which have found relatively small effects for demographic and medical variables related to health anxiety. Overall, these studies have found somewhat weak correlations between significant heath anxiety and being female, middle or older aged, lower education, unemployed, a smoker, and having a comorbid physical condition (Bleichhardt \& Hiller, 2007; Sunderland, Newby, \& Andrews, 2013). Taken together, demographic and medical variables may not be the most robust predictors of health anxiety. 
Another possible explanation is that age contextualizes many of the other demographic and medical predictors. For example, individuals who are younger in age are also more likely to have younger children and to have lived with the knowledge of their Lynch syndrome for less time. In our sample, age was highly correlated with a number of the predictor variables, such as time since cancer or Lynch syndrome diagnosis, having been previously diagnosed with cancer, the total number of cancer diagnoses, the number of first degree relatives diagnosed with cancer, and having younger children ( $r=.45$ to $r=.64)$. Therefore, age may serve as conceptual and statistical proxy for these other non-significant predictor variables.

We had expected younger age to be significantly associated with greater health anxiety, given the established link between younger age and psychological distress within the larger cancer literature. For example, a large cross-sectional study of cancer patients with varied diagnoses found that those who were younger in age exhibited the highest levels of psychological distress (Zabora, BrintzenhofeSzoc, Curbow, Hooker, \& Piantadosi, 2001). Previous researchers have suggested that younger cancer patients may experience elevated distress due to their stage of life and the associated responsibilities. For example, having cancer and undergoing treatment can have a significant impact on one's career, finances, and child-rearing (e.g., Ganz, Desmond, Leedham, Rowland, Meyerowitz et al., 2002; Wenzel et al., 1999), factors which are particularly relevant for younger individuals. Within our sample, those who have been diagnosed with cancer were significantly older (58.74 years) than those who have not (44.46 years). Our findings suggest that younger patients, because they are less likely to have had cancer, worry more about the impact of cancer; therefore leading to elevated health anxiety. 
A second rationale as to why younger individuals experience more health anxiety than their older counterparts relates to previous illness experience. According to the theory of psychological immunity (Henderson, Montgomery, \& Williams, 1972), resilience to adverse life events is developed through repeated exposure to them. As older individuals have a greater likelihood of having experienced more threats to their health, this theory provides a rationale as to why a diagnosis of Lynch syndrome would be less distressing to older individuals. Moreover, within our sample, the older individuals have an increased likelihood of being diagnosed with cancer, therefore giving them experience with this specific health threat. An additional factor is that as people age, they have more exposure to peers and family members who are coping with chronic illnesses. Not only does this intensify the amount of exposure a person has to illness, but it also increases the likelihood of a supportive social network of individuals who have a deeper understanding of the illness-related situation. In contrast, younger patients may not know many people who have faced significant threats to their health, and are less likely to have been diagnosed with cancer themselves, leading them to feel different and isolated. Overall, these findings highlight the importance of monitoring health anxiety, particularly in individuals who receive a diagnosis of Lynch syndrome at a younger age.

Psychological predictors of health anxiety. In examining psychological predictors of health anxiety, Hypothesis $2 \mathrm{~b}$ was partially supported. Specifically, after controlling for age, greater health anxiety was significantly predicted by greater anxiety, greater depression, greater worry interference, and more use of the coping strategy of emotional preoccupation. Contrary to what was hypothesized, more use of distraction based coping 
and less use of palliative and instrumental based coping were not predictive of health anxiety.

As expected, anxiety, depression and worry interference were found to be predictive of increased health anxiety. Previous research has also noted the association between psychological distress, anxiety, depression, and health anxiety in both medical and non-medical samples (d'Agincourt-Canning, 2005; Esplen et al., 2003; Gritz et al., 2005; Hadjistavropoulos et al., 2012; Keller et al., 2002, 2008; Mcallister, 2002; Bettina Meiser, 2005; Rimes et al., 2006; Van Oostrom et al., 2006). It is not surprising that depression and anxiety were related to health anxiety in the present sample, as there is conceptual and diagnostic overlap between these psychological disorders. In fact, in the general population, individuals with health anxiety have been shown to be more likely to experience comorbid disorders such as panic disorder, generalized anxiety disorder, bipolar disorder, and agoraphobia (Asmundson et al., 2001; Sunderland et al., 2013). However, it is clear that health anxiety represents a distinct construct from general anxiety and depression, as evidenced by only moderate levels of correlation between the measures used in this study. Similarly, the observed relationship between worry interference (i.e., the degree to which thoughts about Lynch syndrome or cancer interfered with participant's daily functioning) and health anxiety was also expected. Although there is limited research examining the relationship between these two constructs, prior data show that intrusive thoughts are linked to higher levels of anxiety in women at high risk of developing breast or ovarian cancer (Trask et al., 2001). Additionally, in a study examining health anxiety in individuals "at-risk" for developing cancer (e.g., smokers, X-ray technicians, BRCA-1 and BRCA-2 mutation carriers), 
Shiloh and colleagues (2008) found that worry interference was moderately positively correlated with health anxiety. Overall, the link between anxiety, depression, and worry interference and elevated health anxiety among Lynch syndrome carriers is important as it highlights factors predisposing individuals to increased anxiety about their health, even many years after receiving a diagnosis of Lynch syndrome.

Among our psychological predictors examined, only one of the four coping strategies (i.e., emotional preoccupation) was related to health anxiety. This was an unexpected finding, given that previous research shows health anxiety to be associated with a broad array of coping strategies. For example, in various medical populations, greater use of emotional preoccupation coping and social support and less use of problem-focused coping have been shown to be linked to higher health anxiety (Hadjistavropoulos et al., 2012; Kehler \& Hadjistavropoulos, 2009).

Emotional preoccupation coping contrasts starkly to problem-focused coping strategies, which are aimed at taking control of the situation and actively modifying certain aspects as a method to cope with an illness related problem. Emotional preoccupation, a construct which is closely related to the widely studied emotion-focused coping style, is characterized by focusing on the negative emotional consequences of a health problem, such as being angry, anxious or frustrated because of an illness or injury (Endler \& Parker, 2000). Although emotional preoccupation was the least frequently endorsed coping strategy, our findings suggest that individuals who become preoccupied with the emotional repercussions when faced with Lynch Syndrome experience heightened levels of health anxiety. This result is consistent with the HA model (Warwick \& Salkovskis, 1990) which proposes that an increased focus on the negative 
aspects of a health condition serves to maintain and exacerbate the anxiety an individual experiences. In fact, emotional preoccupation coping has been associated with a variety of negative constructs, such as increased psychological distress, poorer psychological adjustment to illness, depression, state anxiety, greater pain severity, and increased life interference in chronically ill populations (Johnson and Endler, 2002; Endler, Corace, Summerfeldt, Johnson \& Rothbart, 2003; Macrodimitris and Endler, 2001). In general, these results suggest that emotional preoccupation coping is a key factor in understanding health anxiety.

One interesting pattern from our findings is that all of the variables which significantly predicted health anxiety were maladaptive or problematic factors (i.e., depression, anxiety, emotional preoccupation coping, and worry interference). In contrast, the more effective coping styles of palliative or instrumental based coping were not found to be predictive of decreased health anxiety. As previously reported, there is a plethora of research supporting the relationship between these negative predictor variables and overall psychological distress and, conversely, the relationship between effective coping styles and psychological well-being (e.g., Hadjistavropoulos et al., 2012; Kehler \& Hadjistavropoulos, 2009). However, in the literature on coping with medical issues, there are some studies similarly demonstrating that maladaptive coping appears to have a larger impact on psychological distress than more adaptive coping. For example, Vriezekolk and colleagues (2011) found in a systematic review that avoidant-focused coping was longitudinally associated with psychological distress for individuals with rheumatoid arthritis, however, no relationship was found that supported the idea that approach-focused coping decreases psychological distress. It appears that for individuals 
with Lynch syndrome, psychological distress and maladaptive coping behaviours are particularly associated with increased health anxiety, whereas engaging in adaptive coping does not similarly reduce the intensity of health anxiety.

\section{Consequences of Health Anxiety.}

Medical service utilization. Hypothesis 3 examined the relationship between health anxiety and medical utilization among Lynch syndrome carriers. As predicted, higher health anxiety was related to increased medical utilization; however, it appears that this relationship was supported for only specific types of medical visits. Increased health anxiety predicted medical utilization, when measured as a total composite score. However, when examining the type of medical visit separately, health anxiety was found to be a significant predictor of only increased visits to a gastroenterologist and the emergency room. Health anxiety was not a significant predictor of visits to a general practitioner, oncologist, surgeon, cardiologist, gynaecologist, genetic counsellor, or 'other' medical professionals.

We found that our sample made more visits to medical professionals than general medical outpatients in other studies. The average number of medical visits our sample reported (7.06 in a 12-month period) was higher than for general medical outpatients (4.4 visits in a 12-month period; Barsky et al., 1999). This finding may reflect the fact that a large proportion of our sample had been diagnosed with cancer. Indeed, individuals with cancer would have higher medical utilization rates as treatment for cancer commonly require a series of visits to varying medical specialities (e.g., seeing an oncologist, visiting a hospital for chemotherapy appointments, etc.). Additionally, even after remission, cancer patients are followed closely by medical professionals. However, our 
data continue to underscore the impact of health anxiety on use of medical services, as health anxiety predicted medical utilization above and beyond the variance accounted for by a cancer diagnosis. Therefore, it does not appear to be the case that having cancer or having recently been in cancer remission is the complete rationale for increased medical utilization; health anxiety plays a unique and undeniable role.

Within healthy populations, anxiety also has been clearly linked with increased medical utilization (Deacon, Lickel, \& Abramowitz, 2008; Kennedy \& Schwab, 1997), likely due to the distressing physical symptoms common in anxiety (e.g., shortness of breath, chest pain; Fleet, Dupuis, Marchand, Burelle, Arsenault, \& Beitman, 1996). Interestingly, the medical utilization reported by Lynch syndrome carriers is slightly higher than what is referred to as an "inflated utilization rate" by individuals diagnosed with an anxiety disorder (approximately six visits in one year; Deacon et al., 2008; Kennedy \& Schwab, 1997), adding further support for relationship between health anxiety and medical utilization among individuals with Lynch syndrome. This relationship is consistent with the HA model, which suggests that individuals with health anxiety are more likely to perceive harmless physical sensations and other health information as threats (Warwick \& Salkovskis, 1990). These perceptions of threat trigger a cycle of emotional distress, dysfunctional cognitions about the symptom or illness, and increased physiological arousal which causes the individual to engage in behaviours intended to reduce the distress. Although purely speculative, one interpretation of the current findings is that health anxious individuals engage in increased medical utilization in an attempt to reduce their distress by seeking reassurance from a medical professional. 
To definitively answer this question, however, the motivation behind each medical appointment made by patients would have to be assessed.

Notably, there are differences in the type of medical specialist sought out by health anxious individuals with Lynch syndrome, with health anxiety being predictive of increased visits to a gastroenterologist and the emergency room. This pattern has also been observed in previous research which suggests that anxious individuals will seek out different types of medical services depending on the type of physical symptoms they experience and/or perceive as threatening. For example, individuals with panic disorder are more likely to present at the emergency department, cardiology, or a general practitioners office than other medical specialities (Barsky, Delamater, \& Orav, 1999; Deacon et al., 2008; Kennedy \& Schwab, 1997), whereas individual with generalized anxiety disorder report attending significantly more gastroenterologist appointments (Kennedy \& Schwab, 1997).

Given the increased risk of cancer, it is not surprising Lynch syndrome patients would be more likely to seek medical reassurance from the gastroenterologist and emergency department. Most patients with Lynch syndrome attend gastroenterologist appointments at least once every one to two years to undergo colonoscopy to screen for colorectal cancer or polyps (Vasen et al., 2013). Consequently, patients may perceive their gastroenterologist as the medical professional who is most responsible for monitoring their health. The tendency for health anxious Lynch syndrome carriers to seek visits with their gastroenterologist may be reflective of a hypervigilance toward any symptoms they feel are related to problems in their digestive systems. Because colorectal cancer is the most common cancer associated with Lynch syndrome (Vasen et al., 2013), 
and as screenings for colorectal cancer are quite effective at preventing colorectal cancer or detecting colorectal cancer at an early stage (American Cancer Society, 2011), patients' desire to seek reassurance from a gastroenterologist is reasonable.

Our finding that greater health anxiety was predictive of emergency room visits is consistent with a large body of literature demonstrating a strong link between patient distress and emergency room visits. In fact, in Canada, it is estimated that between $25.5 \%$ and $60 \%$ of cases seen in an emergency department are actually non-urgent in nature (Afilalo et al., 2004; Beland, Lemay, \& Boucher, 1998). There are a variety of reasons why non-urgent patients access the emergency department, however, the factors that appear to be most relevant to the current study involve the perception of symptom severity and the accessibility of services. In general, patients who present at an emergency room have higher anxiety levels and are more disturbed by their symptoms than individuals who seek primary care services for the same symptoms (Backman, Blomqvist, Lagerlund, Carlsson-Holm, \& Adami, 2008). From a psychological perspective, seeking emergency department medical services is related to patients' misperceptions of the severity of the problem and their need to obtain reassurance (Afilalo et al., 2004; Guttman, Zimmerman, \& Nelson, 2003; Lega \& Mengoni, 2008; Lewis, Alpert, Lebo, \& Bane, 2006). These features mirror the experiences of individuals with high health anxiety as outlined by the HA model, where patients exhibit a tendency to misinterpret benign physical sensations as dangerous and seek reassurance as a method of coping with this anxiety. Second, the emergency department offers 24hour access and does not require an appointment, meaning that reassurance can be sought in a much quicker and relatively easier fashion than having to wait for an appointment to 
address these concerns in a primary care setting (Lega \& Mengoni, 2008), thus reducing the length of patient distress.

What is more unexpected, however, is that a similar pattern did not occur between health anxiety and visits to gynecologists in our sample. The risk of developing endometrial cancer in female carriers of Lynch syndrome is comparable to the risk of developing colorectal cancer (30-70\%; Vasen et al., 2013), therefore, we anticipated that the same level of hypervigilance and reassurance seeking for colorectal cancer would also be observed for gynecological cancers. The fact that this pattern was not observed may be reflective of the lack of awareness of the risk for gynecological cancers in Lynch syndrome. For example, until recently Lynch syndrome was referred to as "hereditary nonpolyposis colorectal cancer (HNPCC)." The name was changed to Lynch syndrome to reflect that the syndrome is linked to cancers other than colorectal. However, there may be a continued misperception held by patients and physicians that the primary or exclusive concern of Lynch syndrome is colorectal cancer. Furthermore, medical research of Lynch syndrome associated endometrial cancers is far less developed than the research on Lynch syndrome associated colon cancer and clinicians are more likely to emphasize colon cancer screening than endometrial cancer screening options ( $\mathrm{Lu} \mathrm{\&}$ Broaddus, 2005). This overall lack of awareness of Lynch syndrome associated endometrial cancer may help explain why no relationship between health anxiety and visits to a gynecologist was observed.

\section{Limitations of the Present Study}

A number of limitations must be considered when interpreting the present findings. In particular, there are a number of conceptual and design related factors that 
may have impacted the findings and warrant further discussion. Perhaps the most predominant limitation is the use of a cross-sectional research design. To conclusively determine whether the aforementioned medical, demographic, and psychological variables are predictive of health anxiety, a longitudinal design is required. Similarly, a longitudinal study would also be necessary to definitively identify medical utilization as a consequence of health anxiety. Therefore, at present, these findings must be conceptualized as correlates since cross-sectional data cannot be used to infer causality. A second limitation is the extent to which these findings are generalizable. The sample consisted of individuals who had all: 1) sought out genetic testing, 2) previously agreed to be included in the FGICR database, and 3) consented to participate in our current study. As such, it is possible that the results from this sample may be influenced by selection bias and may not be representative of the larger population of individuals with Lynch syndrome. Additionally, our sample was primarily white, highly educated, married or partnered, and employed. Despite these concerns, it is important to note that our sample was found to be comparable with regards to medical and demographic characteristics observed in other research examining Lynch syndrome populations (e.g., Landsbergen et al., 2012; Aktan-Collan et al., 2013). Moreover, the present study had a response rate of $62.7 \%$, which is consistent with the response rate from questionnairebased research that recruits participants from cancer registries (50-65\%; Esplen et al., 2007; Esplen et al., 2001). Unfortunately, data comparing the characteristics of responders to non-responders were not available, thereby limiting our ability to comment on how the self-selection may have further impacted the generalizability of the findings. 
The current sample was very diverse in terms of their demographics, their history of Lynch syndrome, and prior cancer diagnoses. As such, a potential limitation of the current study is the medical variability among Lynch syndrome carriers. For example, the sample varies greatly with respect to the time elapsed since patients learned of their susceptibility to cancer, with some individuals learning of their diagnosis (of either Lynch syndrome or cancer) within the month they completed the questionnaires and others knowing of their susceptibility for 41 years $(M=12.89$ years $)$. Similarly, there is variability with regards to patients' history of cancer, with over half of the sample having had a minimum of one cancer diagnosis in their lives. Therefore, it is possible that the health anxiety of those who have had cancer is more related to their cancer history than to their Lynch syndrome. However, it is important to note that the percentage of individuals diagnosed with cancer in our sample is consistent with the documented likelihood of individuals with Lynch syndrome developing a Lynch syndrome cancer (Jenkins et al., 2006). While it is possible to view the variability in patient medical history as a limitation of the current study, the diversity present in this sample can also be conceptualized as a strength. By including a broader population of Lynch syndrome patients, as opposed to only including newly diagnosed individuals, this study has attempted to capture the experience of a sample that is representative of the general population of individuals with Lynch syndrome.

A final limitation is the validity of some of the self-report measures utilized in the present research. One concern is that the medical service utilization measure was not able to capture whether the visits to medical practitioners was a regularly scheduled appointment or if it was above and beyond a scheduled visit. Having information as to 
whether the appointment was a regular visit would have provided valuable insight into whether patients were being medically compliant versus utilizing excessive medical services. Moreover, another question is whether participants were able to accurately report on the frequency of their medical visits over the past six months. Previous research has demonstrated good concordance between self-reported medical utilization and reports from patients' medical records (Bhandari \& Wagner, 2006; Ritter et al., 2001); however, utilizing provider reports or electronic records could have improved the accuracy of reporting. Similarly, it is important to note that the Worry Interference Scale (WIS; Trask et al., 2001) was modified slightly so that the measure would assess the interference caused by Lynch syndrome as opposed to breast cancer. Although it is unlikely that this change would render the scale invalid, it is a limitation to be considered. 


\section{STUDY II}

\section{Method}

For Study II, in-depth interviews were conducted with a subset of participants who had previously completed Study I. The primary aim of the qualitative component of this study was to examine the impact health anxiety has on parents with Lynch syndrome. Specifically, high and low health anxious parents were interviewed to qualitatively explore for differences in their experience of being a parent with Lynch syndrome, their thoughts and emotions of potentially passing down the genetic mutation to their children, and their perceptions of their children's health.

\section{Research Approach}

The current study utilized qualitative content analysis to examine the differences between high and low health anxiety on the experiences of being a parent with Lynch syndrome. Content analysis is a widely used qualitative research technique that interprets meaning from the content of textual data (Hsieh \& Shannon, 2005). The purpose of content analysis is to provide knowledge and insights about a particular experience. One critical feature of content analysis is the use of categories to analyze the data. Such categories are typically derived from theoretical models a priori, as opposed to being derived from the data itself (Bauer, 2000). For the present study, the HA model (Warwick \& Salkovskis, 1990), in combination with the findings from the literature on genetic predispositions to cancer, was used to guide the development of the interview protocol and was also integral in the coding and interpretation of the data.

Within the present study, as is consistent with content analysis, no formal epistemological lens was utilized. However, as the purpose of the research was to 
understand the experience of the high versus low health anxious parents with Lynch syndrome, some components of a phenomenological lens were used. Generally speaking, phenomenology involves the analysis and description of the shared lived experience of a group of individuals (Flick, 2009). It is focused on the subjectivity of reality, meaning that phenomenology examines how individuals perceive themselves and the world around them (Willis, 2007). Given that the participants in the current study were asked to reflect on their individual experiences, and such experiences were analyzed collectively to examine the shared experience of parents with Lynch syndrome, the research approach drew from a phenomenological perspective.

\section{Participants}

For patients to be considered eligible to participate in Study II, the following criteria were required: 1) The patient must have completed Study I. 2) The patient must have a minimum of one biological child. 3) To allow for extreme case sampling, the patient's health anxiety score, as measured by the SHAI, must be below the $10^{\text {th }}$ or above the $90^{\text {th }}$ percentile of the range of scores obtained for the total sample.

\section{Procedure}

Purposive sampling was used to identify the parents from Study I who reported the highest and lowest health anxiety on the SHAI. These two subgroups were created for the purpose of allowing for comparative analysis of the experiences of parents with high and low health anxiety. In total, 21 individuals were identified whose scores fell below the $10^{\text {th }}$ percentile and 21 individuals were identified whose scores fell above the $90^{\text {th }}$ percentile of scores on the SHAI. The selected participants were then rank ordered according to their scores. Given that previous research has demonstrated clear differences 
in parenting behaviours between mothers and fathers (e.g., Collins \& Russell, 1991, Cowan et al., 1993; Craig, 2006; Klahr \& Burt, 2014), participants were matched in each category (i.e., high versus low health anxiety) on gender, to ensure equal representations for each gender in both categories of anxiety.

Participants were contacted via telephone and informed that they had been selected to participate in a second phase of the research project consisting of an interview. In order to maximize the difference between the high and low anxiety groups, we called participants in descending order for the high anxiety sample and ascending order for the low anxiety sample. If interested, informed consent was obtained and a time was scheduled for the interview to be conducted. As participants lived in a wide range of cities across Ontario, Canada, all interviews were conducted over the telephone.

Interviews were conducted by the primary researcher (JA) and a trained research assistant (NK). The interview was semi-structured and followed an Interview Guide that was created specifically for the current study (see Appendix B). Participants were asked a variety of questions related to their experience of being a parent with Lynch syndrome, their experience of potentially passing down the genetic mutation to their children, and their perceptions of the health and vulnerability of their children. All questions outlined on the Interview Guide were asked, however, follow-up questioning was encouraged where appropriate. Interviews were audio recorded for the purpose of transcription. Interviews took approximately 45 to 60 minutes to complete. Participants were mailed a $\$ 40.00$ gift card for their participation.

In total, 27 individuals were attempted to be contacted for interviews. Four individuals could not be reached and one individual declined participation because of 
time constraints ( $81.5 \%$ response rate). Twenty-two telephone interviews were completed; however, one interview was discarded due to inadequate English language proficiency. As such, a total of 21 interviews were analyzed. Once the interviews were completed, audio recordings from the interviews were transcribed verbatim and uploaded to the qualitative analysis program NVivo 9.0.

\section{Qualitative Analysis}

Participant responses to interview questions were analyzed using a template coding approach. Overall, the template coding scheme was organized into the three main domains identified as relevant to parents who are Lynch syndrome carriers: 1) Perceptions of Self, 2) Perceptions of Passing Down Lynch Syndrome to Child, and 3) Perceptions of Child.

A two-step approach was used to create the coding categories identified in the template coding scheme. In the first step, prior to conducting the interviews and examining the data, a draft codebook was created based primarily on the HA model and findings from previous research examining health anxiety. This draft codebook contained the codes or "themes" that were expected to emerge from the participants" interview responses. The second step of the codebook construction took place after the completion of interviews. The goal of this step was to refine the codebook and ensure that it captured the information participants provided within each main thematic category. No new main themes were added during this step; however, the majority of the subthemes were added during this phase of codebook development. Sub-themes were created by the primary researcher (JA) and a research assistant (NK) independently immersing themselves in the qualitative data. Transcripts were read and reread and key 
points were highlighted. Additionally, notes were made in the margins regarding the researchers thoughts on themes that were identified. After this process was complete, the researchers met to have an iterative discussion about the identified sub-codes. Any disagreements were resolved through discussion. A final round of discussion occurred with the lead researcher and her research supervisor $(\mathrm{TH})$ before the template coding scheme was considered final.

As is consistent with a template coding approach, we allowed for new codes to be developed beyond the initial codes that had been outlined in the first step of codebook construction. More specifically, the template coding scheme remained open to continuous revision throughout the data analysis. See Appendix $\mathrm{C}$ for the final codebook and Figure 1 for a visual representation of the coding scheme.

As the primary researcher (JA) and the research assistant (NK) conducted the interviews and were also the coders of the data, the status of the participant (i.e., high versus low health anxiety) could not be concealed during the coding process. Both coders completed coding for all 21 interviews using NVivo 9.0. Inter-rater reliability, as calculated by percent agreement at the sub-theme level, was acceptable at $82.4 \%$ (Stemler, 2004). Once the coding was completed, the data were organized based on the codes and the participant group (i.e., high versus low health anxiety) into separate tables. This method of organization allowed for differences between the high and low health anxiety groups to be identified. 


\section{Results}

\section{Sample Characteristics}

In total, 21 telephone-based interviews were included in Study II. In total, 11 high health anxiety (5 men, 6 women) and 10 low health anxiety (5 men, 5 women) patients were interviewed. The majority of the interviewees were Caucasian (90.5\%), employed (66.7\%), and college educated or higher (66.6\%). All of the participants were married or partnered (100\%; mean length of relationship 22.2 years, $S D=10.32$ years). The age of participants ranged from 36 to 73 years with a mean age of $51.19(S D=$ 10.64). The mean time since participants had been diagnosed with either cancer or Lynch syndrome was 5.74 years $(\mathrm{SD}=3.83)$. Thirteen individuals $(61.9 \%)$ had been diagnosed with cancer at some point in their lives. Table 11 summarizes the demographic and medical information for the individuals included in Study II and compares the high and low health anxiety groups.

On average, participants had 2.38 children $(\mathrm{SD}=1.16$; range $1-6)$ and $52.4 \%$ of participants had at least one child under the age of 18. In total, the parents who were interviewed had a summed total of 50 children. Twenty-nine children were over the age of 18 (the typical age where genetic testing for Lynch syndrome is available). Out of the 29 children presumed eligible for genetic testing, 17 (58.6\%) had received genetic testing for Lynch syndrome. Of those individuals who received genetic testing, nine children $(52.9 \%)$ were found to be carriers of Lynch syndrome. The aforementioned frequencies are broken down by high versus low health anxiety groups in Table 12. 

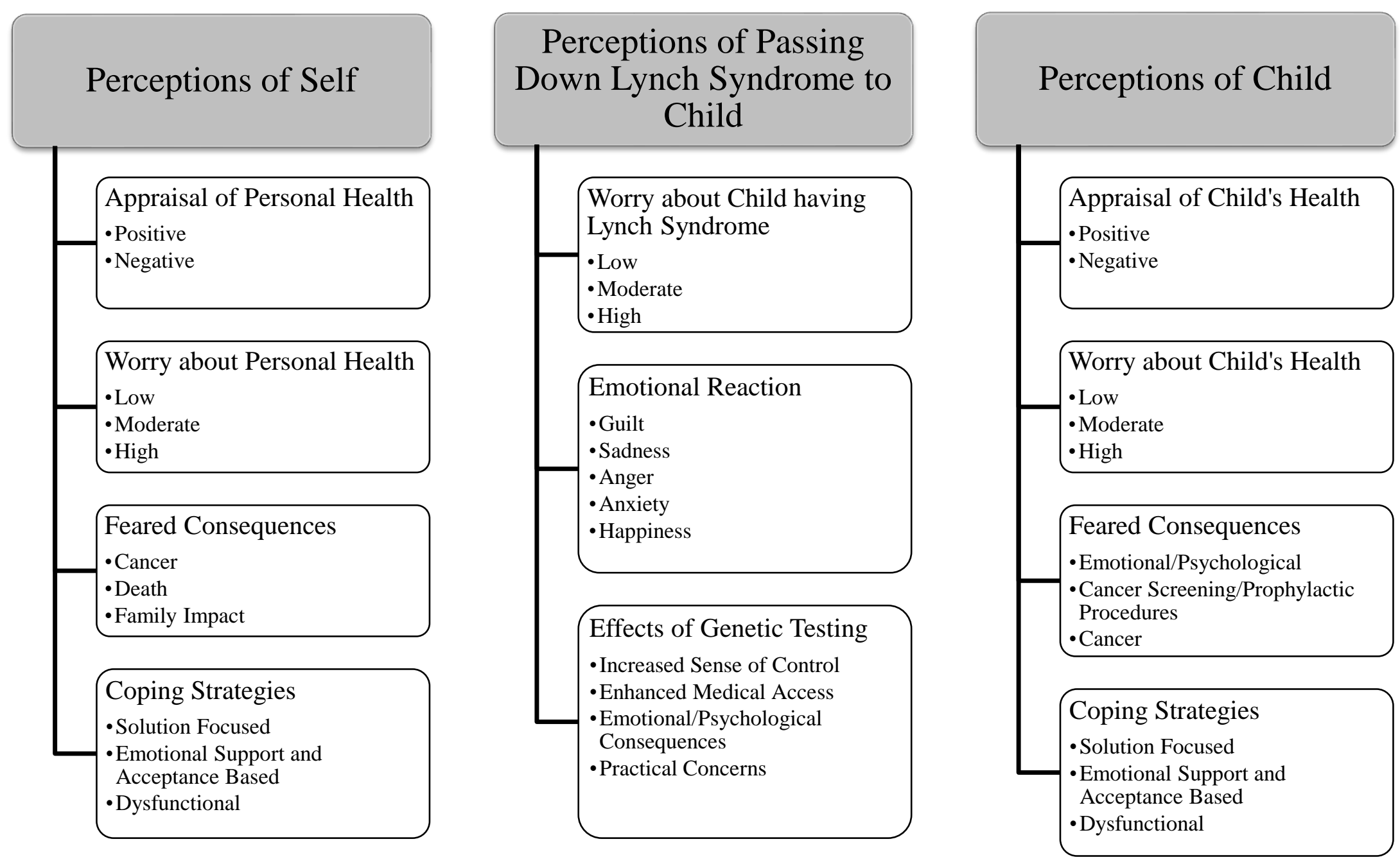

Figure 1. Visual representation of the Study II coding scheme 
Table 11

Study II Sample Demographics and Medical Characteristic $(N=21)$

\begin{tabular}{|c|c|c|c|}
\hline & $\begin{array}{l}\text { Low Health Anxiety } \\
\text { Group } \\
\underline{(n=10)}\end{array}$ & $\begin{array}{l}\text { High Health } \\
\text { Anxiety Group } \\
\quad \underline{(n=11)}\end{array}$ & Total $(N=21)$ \\
\hline Variable & $M(S D)$ & $M(S D)$ & $M(S D)$ \\
\hline Age (years) & $53.2(9.8)$ & $49.36(11.5)$ & $51.2(10.6)$ \\
\hline
\end{tabular}

Gender

$\begin{array}{llll}\text { Male } & 5(50.0) & 5(45.5) & 10(47.6) \\ \text { Female } & 5(50.0) & 6(54.5) & 11(52.4)\end{array}$

Ethnicity

\begin{tabular}{cccc}
\multicolumn{1}{c}{ White } & $10(100)$ & $9(42.9)$ & $19(90.5)$ \\
Asian & $0(0.0)$ & $2(9.5)$ & $2(9.5)$ \\
$\begin{array}{l}\text { Employment } \\
\text { status } \\
\text { Full-time }\end{array}$ & $7(70.0)$ & $4(36.4)$ & $11(52.4)$ \\
Part-time & $1(10.0)$ & $2(18.2)$ & $3(14.3)$ \\
Retired & $2(20.0)$ & $1(9.0)$ & $3(14.3)$ \\
Disability & $0(0.0)$ & $1(9.0)$ & $1(4.8)$ \\
Unemployed & $0(0.0)$ & $3(27.3)$ & $3(14.3)$ \\
$\begin{array}{l}\text { Average Annual } \\
\text { Income } \\
0-40,000\end{array}$ & $4(40.0)$ & & $5(23.8)$ \\
$\begin{array}{l}40,000- \\
75,000\end{array}$ & $5(50.0)$ & $1(9.0)$ & $9(42.9)$ \\
$>75,000$ & $1(10.0)$ & $4(36.4)$ & $6(28.6)$
\end{tabular}

Education

$\begin{array}{lccc}\begin{array}{l}\text { High school or } \\ \text { below }\end{array} & 3(30.0) & 3(27.3) & 6(28.6) \\ \text { Some college } & 1(10.0) & 0(0.0) & 1(4.8)\end{array}$


Table 11 (continued)

Phase II Sample Demographics and Medical Characteristic $(N=21)$

\begin{tabular}{|c|c|c|c|c|c|c|}
\hline \multirow[b]{2}{*}{ Variable } & \multicolumn{2}{|c|}{$\begin{array}{l}\text { Low Health Anxiety } \\
\text { Group } \\
\underline{(n=10)}\end{array}$} & \multicolumn{2}{|c|}{$\begin{array}{l}\text { High Health } \\
\text { Anxiety Group } \\
\quad \underline{(n=11)}\end{array}$} & \multicolumn{2}{|c|}{$\underline{\text { Total }}(N=21)$} \\
\hline & $n(\%)$ & $M(S D)$ & $n(\%)$ & $M(S D)$ & $n(\%)$ & $M(S D)$ \\
\hline College & $5(50.0)$ & & 7 (63.6) & & $12(57.1)$ & \\
\hline $\begin{array}{l}\text { Graduate/ } \\
\text { professional }\end{array}$ & $1(10.0)$ & & $1(9.0)$ & & $2(9.5)$ & \\
\hline $\begin{array}{l}\text { Relationship } \\
\text { Status }\end{array}$ & & & & & & \\
\hline $\begin{array}{l}\text { Married/ } \\
\text { Partnered }\end{array}$ & $10(100)$ & & $11(100)$ & & $21(100)$ & \\
\hline $\begin{array}{l}\text { History of cancer } \\
\text { diagnosis }\end{array}$ & $8(80.0)$ & & $5(45.5)$ & & 13 (61.9) & \\
\hline $\begin{array}{l}\text { Age at first cancer } \\
\text { diagnosis (years; } n \\
=13 \text { ) }\end{array}$ & & $43.1(8.3)$ & & $43.4(6.1)$ & & $43.1(8.3)$ \\
\hline $\begin{array}{l}\text { Total number of } \\
\text { cancer diagnoses } \\
(n=13)\end{array}$ & & $1.4(0.6)$ & & $1.6(0.9)$ & & $1.4(0.7)$ \\
\hline $\begin{array}{l}\text { Recurrence of } \\
\text { same form of } \\
\text { cancer }(n=13)\end{array}$ & & $0.2(0.4)$ & & $0.2(0.4)$ & & $0.2(0.4)$ \\
\hline $\begin{array}{l}\text { Time since first } \\
\text { cancer diagnosis } \\
\text { (years; } n=13 \text { ) }\end{array}$ & & $13.1(8.5)$ & & $10.4(4.8)$ & & $12.1(7.2)$ \\
\hline $\begin{array}{l}\text { Time since } \\
\text { diagnosed with } \\
\text { Lynch syndrome } \\
\text { (years; } n=14 \text { ) }\end{array}$ & & $5.3(2.7)$ & & $6.1(4.7)$ & & $5.74(3.8)$ \\
\hline $\begin{array}{l}\text { Time since } \\
\text { awareness of } \\
\text { cancer } \\
\text { susceptibility } \\
\text { (years; } \mathrm{n}=17 \text { ) }\end{array}$ & & $12.3(8.3)$ & & $9.0(5.2)$ & & $10.7(7.0)$ \\
\hline
\end{tabular}




\section{Overview}

As previously discussed, the template coding scheme was organized into the three main domains thought to be particularly relevant to parents who are Lynch syndrome carriers: 1) Perceptions of Self, 2) Perceptions of Passing Down Lynch Syndrome to Child, and 3) Perceptions of Child. The Perceptions of Self category focused on how the patient viewed their health and future and examined the ways in which their anxiety about Lynch syndrome has impacted them. This category contained four main themes (Appraisal of Personal Health, Worry about Personal Health, Feared Consequences, and Coping Strategies) and had 11 sub-themes. ${ }^{3}$ The Perceptions of Passing Down Lynch Syndrome to Child category examined the feelings and concerns parents had regarding their children inheriting Lynch syndrome. This category was comprised of three main thematic categories (Worry about Child having Lynch Syndrome, Emotional Reaction, and Effects of Genetic Testing) and had 12 sub-themes. Finally, the Perceptions of Child category examined parents' evaluation of their children's health and the impact of this worry. This category contained four themes (Appraisal of Child's Health, Worry about Child's Health, Feared Consequences, and Coping Strategies) and had 11 sub-themes.

\section{Main Findings}

Domain 1: Perceptions of self. The Perceptions of Self category focused on how the patient viewed their health and future and examined the ways in which their anxiety about Lynch syndrome has impacted them. This category contained four main themes (Appraisal of Personal

\footnotetext{
${ }^{3}$ One additional code was originally included in the codebook. Under the domain of Perceptions of Self was the code entitled "Parental Self-Concept" which aimed to identify changes in the way participants viewed themselves and viewed their role as parents since being diagnosed with Lynch syndrome. However, during the interview participants were unable to articulate their answers to this line of questioning and no meaningful data were obtained. As such, this code has been omitted from the final codebook presented in the current study.
} 
Table 12

Demographic and Medical Information about the Children of Study II Participants

\begin{tabular}{lccc}
\hline & $\begin{array}{c}\text { Low } \\
\text { Health } \\
\text { Anxiety } \\
\text { Group } \\
(n)\end{array}$ & $\begin{array}{c}\text { High } \\
\text { Health } \\
\text { Anxiety } \\
\text { Group } \\
(n)\end{array}$ & $\begin{array}{c}\text { Total } \\
(n)\end{array}$ \\
Variable & 26 & 24 & 50 \\
\hline Total number of children & 18 & 11 & 29 \\
Total number of children over age 18 & 9 & 8 & 17 \\
Total number of children tested for Lynch syndrome & 6 & 3 & 9 \\
Total number of children diagnosed with Lynch syndrome & & & \\
\hline
\end{tabular}


Health, Worry about Personal Health, Feared Consequences, and Coping Strategies) and had 11 sub-themes.

Appraisal of personal health. The Appraisal of Personal Health code captured participants' descriptions of their health, their perceived vulnerability to illness, and any comparisons between how they viewed their health versus their peers. Overall, participants tended to describe their health as quite positive, with only a few individuals describing their health as poor. Interestingly, very few of the participants specifically referenced their diagnosis of Lynch syndrome when appraising their current health. In comparing the high versus low health anxious individuals, no differences were noted between the patients with the highest and lowest health anxiety. Instead, participants from both groups tended to focus their evaluations on their current medical symptoms, or lack thereof:

"I think I'm very healthy. I actually just had my physical yesterday and got green lights everywhere, like I said because I'm doing all my screening I'm more aware of my health and trying to stay fit, and especially for my kids as well. So yeah, I can say I'm a relatively healthy person." (Low health anxiety male)

"I have no problems with heart, I have no problems with the lungs, I have no problems with my physical conditions. No, I'm - I would say that I'm a very healthy person." (High health anxiety male)

"I have other ailments like fibromyalgia. The neuropathy because of the fibromyalgia leaves me in a lot of pain the majority of the time, the severity of it changes." (High health anxiety female)

Worry about personal health. Participants were asked to describe the degree to which they worry about their health. As expected, differences in the level of worry were observed between the high and low health anxiety groups. Within the low health anxiety group, the majority of individuals indicated that they do not worry about their health, a smaller proportion stated that they worry a moderate amount, and one individual indicated they worry nearly 
constantly. In general, it appears that the low health anxiety individuals worry infrequently about their health. When they do worry, the thoughts tend to be triggered by external cues and reflect more of an awareness of their health than a worry, per se:

"It's always a bit of fear when you go for a check-up, for colonoscopy, it's normal to have a bit of fear." (Low health anxiety male)

"Not very often. I know I have my colonoscopies every six months, but I don't worry about it. If they find something, they find it and it's early detection so we can do something about it but it's not daily." (Low health anxiety female)

"I think I'm always consciously, not worrying, but aware of any changes in my body. Anything, I'm always paying attention." (Low health anxiety female)

In contrast, within the high health anxiety group, the largest proportion of individuals stated that they worry about their health a great deal, a smaller amount of participants indicated that they worry moderately, and two individuals stated that they do not worry about their health at all. The following quotation from a high health anxiety male typifies the constant worry and hypervigilance reported by many individuals with high health anxiety:

"I worry daily... When I'm feeling different, when my body is feeling a little different. I'm always, my mind always goes to cancer. For some reason, like if my stomach, or if I'm not feeling the same one day, if my stomach is bothering me then I would look at think, 'is that?' or 'should I go for a test?' 'should I ask for another test?'." (High health anxiety male)

Feared Consequences. Participants were asked the very general question, "What about Lynch syndrome are you most worried about with regards to the future?" to assess their feared consequences of this health condition. Participants spontaneously generated three categories that reflected their feared consequences of Lynch syndrome: 1) Cancer, 2) Death, and 3) Family Impact. Interestingly, participants in both the high and low health anxiety groups discussed all three of these categories, however, the high health anxiety group's worries were much more extreme, and they spoke about these concerns more frequently. 
Cancer. When discussing a fear of developing cancer, the low health anxiety group tended to note the possibility of being diagnosed with cancer as a potential future outcome, however, they did not dwell on the consequences of such diagnosis and viewed the diagnosis as manageable:

"[The thought of getting cancer], it's always there, it's always a concern. But if I'm feeling good I just go with the flow." (Low health anxiety male)

"And besides that, if I do have a recurrence of cancer if it's in the colon at least it's in a place that you can remove it. Rather than the lymph nodes or the bladder or things like that where it's much more serious. So it's best to catch early anyways." (Low health anxiety male)

In contrast, when highly health anxious patients spoke of their fear of developing cancer,

they had a tendency to focus on a specific type of cancer or on the prognosis:

"Well I worry about the breast cancer. That is always a worry, I worry about that more than I worry about the colon cancer. I think my - I think how I view it is the colon cancer, it doesn't feel as dangerous for me. I think it's a, with early detection it's a very curable disease. The breast cancer that I had was, it was - I don't know if you're familiar, but it was inflammatory breast cancer, so the outlook was pretty bleak." (High health anxiety female)

Death. Similar to the patterns noted when participants talked about their fear of developing cancer, when discussing the perceived consequence of dying from cancer, the low health anxiety group noted this fear in a brief and hypothetical manner; as a fear they may have thought of but not in a concrete or immediate way:

"Yeah I guess obviously I'd be worried about not surviving." (Low health anxiety male) Whereas the high health anxiety group described their fears of death in greater detail and spoke as if death from cancer was an inevitable fact:

"Oh absolutely. In my mind I will die of cancer. I will get cancer again...I mean I'm just not looking forward to dying of cancer." (High health anxiety male) 
"I just, I don't want to die yet. I'm not scared to die but I just - I feel like I have a life to live and I want to die when I'm old." (High health anxiety female)

"When you're diagnosed with something like Lynch syndrome you have to get used to the idea of living with it because there was actually a period of time where I had started planning my funeral. In the beginning I was preparing to die not to live." (High health anxiety female)

Family impact. Interesting differences emerged when patients with high and low health anxiety described the perceived consequences of their Lynch syndrome on their family. When discussing the worries with regards to the impact Lynch syndrome could have on loved ones, the high health anxiety group primarily focused on the emotional impact their future death would have on family members, particularly children:

"I just hope and pray that I'm here for my kids for as long as I can. I've known and lost people to cancer and young children and I see what happens to their families and that scares me." (High health anxiety female)

"I mean as horrible as this is to say, my husband will be completely fine without me, it's my kids that need me. They need me. They need a dad and they need their mom." (High health anxiety female)

"And the fact that I have a child, you know I waited a long time before having a child, I was 33 when I had my son so... He's my life, he's my motivation, he's what gets me through this because obviously the things that you think about when you're ill or when you worry about becoming ill are you know, I have a kid, I don't want to leave him behind, he can't be without a mother. And that's what gives you strength is I refuse to let him grow up without a mother. So you play little games in your mind, like when I was diagnosed I kept saying oh god, let me live until at least he finishes school. He's not an academic, he struggles in school. So you know, you play little head games with yourself. So it's almost like you give your mind these little timelines that you're going to live by." (High health anxiety female)

The low health anxiety group, however, tended to focus on the practical day-to-day impact they believed Lynch syndrome would have on their family. In particular, these patients tended to focus on the financial consequences of death or cancer: 
"Yeah I guess obviously I'd be worried about not surviving but I think I'm more worried about how - it just changes the dynamic of your family and I guess because, I mean I work in a job where if I can't work, I don't have disability any longer. Whereas when I was 30 I had disability. So I guess my concern is more on the financial side of things and being able to provide for my family if I was sick." (Low health anxiety male)

"One thing I'm thankful for is I have excellent death benefits and pension at work. So I know that if anything happened to me my wife and kids would be taken - would have enough money to get, to live fine without me. So that really helps. If I didn't have that I think I would worry a lot more but I knew that if something happened to me they would carry on. Of course they would miss me but at least they'd be okay financially. And we're close with our family so there's lots of people around to give support that way as well." (Low health anxiety male)

Coping Strategies. Parents were asked to report on the behaviours or strategies they engage in as a method of managing their anxiety regarding their personal health. These coping strategies were grouped into three main categories based on Carver's (1997) coping framework: 1) Solution Focused Coping (e.g., becoming informed about Lynch syndrome, making healthy lifestyle changes, seeking instrumental support), 2) Emotional Support and Acceptance Based Coping (e.g., seeking emotional support from loved ones, positive reappraisal of the situation, acceptance of the situation), and 3) Dysfunctional Coping (e.g., denial, avoidance, rumination, self-blame).

Solution focused coping. Both the high and the low health anxiety groups reported engaging in solution focused coping strategies to deal with their health concerns more than any other form of coping. Notably, the examples of solution focused coping strategies were extremely similar between individuals with high and low health anxiety. In particular, both groups discussed utilizing solution focused coping strategies such as becoming informed about Lynch syndrome, making healthy lifestyle changes, attending regular cancer screening appointments, and seeing a physician when aware of health changes: 
Solution focused coping: Information seeking.

"I think it was much easier [for me to cope] because I'm an educated woman so I looked it up and found the information I needed." (High health anxiety female)

"Well I did a lot of research. And I figured that the more I know, the less I worry... The more knowledge I have - I think people fear the unknown more than anything. And their own imagination can make things worse than they are. But if I know the situation, it's not as bad as I thought it was then I feel a lot better." (Low health anxiety male)

Solution focused coping: Making healthy lifestyle changes.

"I don't know, [I've stopped] the reckless lifestyle - you know drinking and smoking - I was a bit of a social smoker, which had to completely stop, you know, even drinking I just knew that that couldn't be a part of my life anymore." (High health anxiety female)

“What do I do to cope? Maybe just that I make sure I'm healthier. I've quit smoking and done all kinds of things to try to make myself as healthy as I can within my own power." (Low health anxiety female)

Solution focused coping: Attending regular cancer screening appointments.

"I go for my testing when I'm supposed to go then it's frees my mind of any other thought where, if I didn't go for the testing I would kind of wonder what was going on." (High health anxiety male)

"So I followed that suggestion and luckily I receive a colon scope every year since then. And even though it's not a comfortable procedure, but it kind of gives me a good peace of mind that I'm clean every year. So from then on it calmed me down a lot since I know that I'm colon cancer free every year." (High health anxiety male)

"Yeah so basically just knowing that I'm getting checked regularly, and I'm, because I had colon cancer, I'm kind of aware of some of the symptoms behind what I experienced in the past so I just sort of keep that in mind and keep an eye out. Basically just be aware of how I'm feeling." (Low health anxiety male) 
Solution focused coping: Physician visits.

"I tend to wait a while and see if [the physical symptom] goes away and then I stop worrying about it. If it lasts more than a few days I get really worried and go to my doctor." (High health anxiety female)

"Well I'm trying to be proactive. And if there is a problem I'm trying to do whatever I can to make it right, so to speak. Like maybe I go see a doctor. So by doing that I feel I'm containing the problem, I'm on top of the problem." (Low health anxiety male)

Emotional support and acceptance based coping. Similar to the findings for solution focused coping strategies, individuals in the high and low health anxiety groups spoke with equal frequency about using emotional support and acceptance based coping strategies. Individuals in both groups discussed the benefits of receiving emotional support from family and friends, using an acceptance based stance regarding their Lynch syndrome, and attempting to positively reappraise their diagnosis of Lynch syndrome:

Emotional support and acceptance based coping: Emotional support.

"I talk to my husband and that helps a lot for sure. Not anyone else. When I was first diagnosed I talked to my girlfriends but not on an ongoing basis. Just my husband and if I have any questions I'll go to my doctor but not my family. I don't want them stressed out. But talking to my husband and that makes me feel better." (high health anxiety female)

"The one thing I have is a very strong network of friends who I can always count on. Even when I got sick when the boys were little kids, my friends took over and I didn't need to worry about the children. So I was lucky in that sense, not only with my friends but the community itself and the school. There was a lot of support, and I still have that support today. If I am feeling down, there is always someone I can go to." (high health anxiety female)

"I got friends, I got family, I got lots of people in my life. That helps." (Low health anxiety male)

Emotional support and acceptance based coping: Acceptance of Lynch syndrome. 
"I guess the biggest thing that I've learned is that the hardest thing to learn is that I can't control this, like I can't change it. There are some things as you're an adult you realize you can't change; you have no control over it. Yes I cannot smoke, and drink a little bit and put flax in my - all that stuff, but there's no guarantee that I'm not going to get it." (High health anxiety female)

"Not worrying about things that I cannot control. If I can control something, I do what I can to fix it and don't - I won't obsess over something if I can't fix it." (Low health anxiety male)

"But I really don't worry because it doesn't make any difference whether you worry or not. It's probably worse off for you if you do." (Low health anxiety female)

Emotional support and acceptance based coping: Positive reappraisal of Lynch syndrome.

"I mean I could be worse. I always try to look on the bright side. There are people with harder hardships than I have. I just cope with it. Everyone has things they have to deal with in life and this is mine." (High health anxiety female)

"I think at the onset it's like anything else, I don't know the words, I mean you find out you have this Lynch syndrome, and it does change your world but you get back into the way you were and you start eating healthy and you start exercising and you do all these wonderful things and you go back to the way everything was. And you kind of put it on a back burner. And now I don't even think about it." (Low health anxiety female )

"I mean I've always just felt that you can't dwell on those negative feelings because it doesn't help you. I mean like when I look back to when I was going through chemo, I had to have preventative chemotherapy, when I was going through that I look back at that like it was an opportunity where I was 30 years old, at the time I had three young kids, no four young kids. I used that time to spend with my family, which most people never have the opportunity to do in regards to we went camping, we did different things that normally you would never be able to do as a young family because your busy working and doing. I was off work for two months, so it was a good opportunity to spend time with the family." (Low health anxiety male)

The only difference between the high and low health anxiety individuals was that only the high anxiety Lynch syndrome carriers discussed using prayer and faith as a method of coping with their worries: 
"I pray about it... Well, prayer is a big thing for me." (High health anxiety female)

"I went through seven years of infertility, I wasn't supposed to have any kids and the way I look at it God wouldn't take me away from my boys after all that time. My faith is strong." (High health anxiety female)

Dysfunctional coping. The biggest difference observed between the high and low health anxiety groups was with regards to their use of dysfunctional coping strategies. In fact, no items were coded as dysfunctional coping for the low health anxiety group. For the high health anxiety individuals, although dysfunctional coping strategies were the least frequently endorsed coping strategy, the majority of participants indicated they utilized dysfunctional coping behaviours. The most common examples included catastrophizing about the future, avoidance, and rumination:

Dysfunctional coping: Catastrophizing.

"I'm more of a realist, I look to the worst possible scenario. I guess that's my coping mechanism, because if the worst doesn't happen I feel like I got a bonus." (High health anxiety female)

Dysfunctional coping: Avoidance.

"Yes, whenever there's a symptom, any kind of symptom there's a-it brings it to the forefront of your mind. And you're always wondering. You jump to conclusions. Your mind takes over, it's - sometimes you think a self-fulfilling prophecy. So that's why you really try to get it out of your head because I don't want to think about it too much so I do everything in my power not to think about it." (High health anxiety female)

"Yeah, I spend a lot of time trying not to think about it. And then talking about it just forces me to think about it, so I avoid that." (High health anxiety female)

Dysfunctional coping: Rumination.

"What makes it a lot worse? If I keep dwelling on it, day after day after day. I don't do that so much now but when I was younger, I think when I first found out I think I probably festered about it and there's dwelling on it for days. And I think I was isolated, 
like I was a new mom, I didn't - there's so many different factors when I first found out that made it really, not a good time for me to... I don't honestly think that I should have been given the results when I was pregnant, but anyway. I think that because I was - it's different then and now. My worrying then is different than how I deal with it now. Before I would dwell, I was isolated, I had this newborn, I kind of sheltered myself. And I don't think that was good." (High health anxiety female)

Domain 2: Perceptions of passing down the gene to child. The Perceptions of Passing Down Lynch Syndrome to Child category examined the feelings and concerns parents had regarding their children inheriting Lynch syndrome. This category was comprised of three main thematic categories (Worry about Child having Lynch Syndrome, Emotional Reaction, and Effects of Genetic Testing) and had 12 sub-themes.

Worry about child having Lynch syndrome. Participants were asked to describe the degree to which they think about Lynch syndrome being passed down to future generations (i.e., children, grandchildren). Overall, it appears that parents with Lynch syndrome occasionally think about their children having Lynch syndrome. Although high and low health anxiety patients differed with regards to their worry about their personal health, the high and low health anxious parents with Lynch syndrome reported a comparable frequency of thoughts about passing down the gene to their children. Only one high health anxiety individual stated that she thinks about her children having Lynch syndrome on a daily basis. In general, parents expressed a tendency to think about the possibility of their children having Lynch syndrome when they are triggered by external cues such as attending a cancer screening appointment or hearing about genetic testing:

"[Do I think a lot about my kids having Lynch syndrome?] No not often, it seems when it does come up it comes up at the time I go for my colonoscopy." (High health anxiety female) 
"I think about it when, maybe at a family gathering it could come up in a discussion or something or if one of the kids is going to have a colonoscopy or something like that and I think that will come up more so as my kids reach that age. It's something that even my younger kids know, once they hit 20 they'll have to get checked out and things like that." (Low health anxiety male)

Emotional Reaction. This category captured the emotional reaction of parents to the idea that their children are carriers of Lynch syndrome. Parents of children who had previously been tested for Lynch syndrome were asked to recall how they reacted to the results from the genetic test, whereas parents whose children had not received genetic testing for Lynch syndrome were asked to predict how they would feel if they learned that their child was a carrier. Again, both the high and low health anxious parents responded in a similar manner. The two most prominent emotions to learning that a child was a Lynch syndrome carrier were guilt and sadness:

Guilt.

"Just guilt, that's the only emotion I feel... If they were to get [Lynch syndrome] because it came from me. When you're a parent, they're your world and when you become pregnant the only thing you can think about is whether the baby is healthy." (High health anxiety female)

"So I have a lot, a tremendous amount of guilt. That if I could take [the Lynch syndrome] away, I'd take it away in a heartbeat." (High health anxiety female)

"That's right but you feel guilty because it's your body that's failed to pass on a good gene. No matter what you do with them as kids, no matter how you fed them or how well you took care of yourself while you were pregnant... I would feel really guilty and I would be afraid that when they had children they might pass it on to their kids. You never want your kids to be sick because of you." (Low health anxiety female)

Sadness.

"Ifeel sorry for her because - sad that she has this defect and has to manage it. She has been going for colonoscopies since she was 20 so I guess she's had 2 or 3 of them now so far but - so that's a burden, things have been a rollercoaster." (High health anxiety male) 
"Well of course I would be sad that it happened again. Just the same as I was with my other children." (Low health anxiety female)

The final two emotions that were noted by participants were anxiety and anger. Not surprisingly, the high health anxiety group spoke more frequently of experiencing anxiety when thinking about [the possibility of] passing down Lynch syndrome to their children, using words such as "worried," "anxious," "scared," and "terrified" to describe their emotional reactions. Anger was an emotion that was rarely discussed; however, this emotion was mentioned by two high health anxious individuals and one low health anxious participant.

Effects of genetic testing. Parents were asked to describe the predicted or actual benefits and/or consequences of having their children genetically tested for Lynch syndrome. Parents discussed factors related to having an increased sense of control, medical care, psychological consequences, and practical day-to-day concerns.

Increased sense of control. The most commonly described benefit of having children genetically tested was an increased sense of control over the child's health and well-being. No differences were observed between high and low health anxious individuals with regard to this factor. In fact, participants in both the high and low health anxious groups stated that "knowledge is power":

"Power is knowledge. Or knowledge is power. So he would have to - he'd have to use that diagnosis to steer his life. He'd have to realize that having that predisposition, there comes a responsibility. He needs to be responsible with his life and his health; he can't just be like your typical teenagers and run and drink and party. And if he ever smokes, realize like - or not kid himself that he 's immune to it." (High health anxiety female)

"I think I looked at it more as a great opportunity for my kids to know, to do the testing and be a little bit more proactive for that." (Low health anxiety male) 
"So [genetic testing] was very informative. It's really good we know now. I'm really glad we know, because it opens a bunch of windows to see in and think this is where we go from here." (Low health anxiety female)

Enhanced medical access. Similarly, no differences were observed between high and low health anxiety groups with regards to their description of the medical or health related benefits to knowing about a child's Lynch syndrome carrier status. Parents in both groups spoke about the benefits of having access to regular cancer screenings, medical professionals, and research that can benefit them from a medical standpoint:

"This is now something - another health issue that she has to deal with - I wouldn't say continuously, but going forward... Knowing just makes for much more easier access to the tests and to the facilities that - the clinic, and Mount Sinai, the researchers." (High health anxiety male)

"Actually now that I think about that, my diagnosis and my son's diagnosis has given me access to have colonoscopies every year to two years where most people they won't do that, and they won't do them for young people because there's no reason to have them done. So in that sense maybe I am glad we had it done and that my doctor is aware he knows to check this and when to go into it further. If I wasn't diagnosed he might just brush it off and say 'that's not a big deal.', (Low health anxiety female)

Emotional/psychological consequences. Differences between the high and low health anxious parents did, however, emerge with regards to their descriptions of the emotional and psychological impact of having their children tested for Lynch syndrome. Specifically, the high anxiety parents spoke more frequently about the emotional changes that occurred following testing and also more frequently noted worries about their children obtaining insurance in the future. With regards to emotional consequences of testing, the high health anxiety parents tended to focus on their concerns about their child's ability to deal with the emotions that would come with a diagnosis of Lynch syndrome: 
"[I want him to wait until he is older to be tested] so that he's better equipped to deal with it... Yes, emotionally. I don't think emotionally now, or as a teenager, he would realize the ramifications of it. I think it's just, once he's fully matured and all finished growing, then you can tackle that kind of knowledge." (High health anxiety female)

"If it was a positive it could be really stressful. I don't think they're ready for it yet." (High health anxiety female)

Practical concerns. Similar to the differences noted with regards to the emotional and psychological impact of having children tested for Lynch syndrome, the high and low health anxiety parents differed with regards to the practical concern that that their children would not be able to obtain life insurance in the future. This concern was spontaneously generated by seven parents in the high health anxiety group. In contrast, similar concerns were only noted by two low health anxious individuals.

Domain 3: Perceptions of child. The Perceptions of Child category examined parents' evaluation of their children's health and the impact of this worry. This category contained five themes (Appraisal of Child's Health, Worry about Child's Health, Feared Consequences, and Coping Strategies) and had 11 sub-themes.

Appraisal of child's health. Similar to the findings for appraisals of parents' perceptions of their personal health, no differences were observed between the patients with the highest and lowest health anxiety on their perceptions of their children's health. Overall, participants tended to describe their children's health as quite positive, with only a few instances of parents describing health problems. Consistent with responses to their perceptions of their own health, no parents mentioned Lynch syndrome when describing their children's health. In the few instances that parents described their child's health in a negative context, their description was 
related to discrete problems that their children experience (e.g., allergies, growing pains, obesity, psoriasis).

Worry about child's health. Participants were asked to describe the degree to which they worry about their child's health. Contrary to expectations, parents in the high and low health anxiety groups reported a similar frequency of worry about their children's health. Nearly all parents reported that they worry a moderate amount about their children's health. This diverges from our results showing that high health anxiety parents worried substantially more about their personal health than the low health anxiety parents. For the majority of parents who reported worrying a moderate amount about their children's health, their worries tended to focus on their children's current health status:

"Yes, at times [I worry about my children's health]... It is more when there was an odd kind of thing physically happening to them that I don't understand." (High health anxiety female)

"Of course I worry about my son's health, but at the present moment I don't think that he has any... well he has some migraine headaches which kind of attack him every now and then but that's something else... that's nothing to do with the genes for colon cancer." (High health anxiety male)

"Recently my daughter has started to get headaches. I had a relative who had brain cancer. So they sent her for an MRI and everything turned out fine but until you get that final notice... I worry because I know she is [worrying]." (Low health anxiety male)

Although only two high health anxiety and two low health anxiety parents reported experiencing excessive worries about their children's health, the content of these worries was quite different from those who reported moderate worries. Specifically, the worries tended to be future oriented and demonstrated catastrophic thinking patterns:

"I hate that my mind goes there, but he's young, he's growing, he's an adolescent. He has aches and pains all the time. Sometimes I think he's a little hypochondriac because he's always complaining. So when he's complaining, [cancer] is the first thing that bounces into my mind and I question him. Okay, where's the ache or pain, [Name]? How long have you had it? I go through the little list of questions that's in my mind like my little 
two week timeline. So I grill him a little deeper than probably most parents would because I am so aware of what we are predisposed to." (High health anxiety female)

"With [regards to] myself I'm not anxious. With my family members I am. With my kids especially, the one who was really sick especially, I worry constantly. When something bad does happen I elevate from regular/normal to panic instantly. I'm not an anxious person on a regular basis but I'm nervous. Does that make sense?" (Low health anxiety female)

Feared consequences. When parents were asked to discuss their main fears regarding their children developing Lynch syndrome, they spontaneously generated three categories they worried about most: 1) Emotional/Psychological, 2) Cancer Screening/Prophylactic Procedures, and 3) Cancer.

Emotional/psychological. Differences emerged between the high and low health anxiety groups with regards to their concerns about the emotional and psychological impact being diagnosed with Lynch syndrome would have on their children. Specifically, only the high health anxiety group discussed emotional and psychological outcomes as a feared consequence of their children having Lynch syndrome. High health anxious parents reported that they feared that their children would have to live with the worry, anxiety, and uncertainty that often accompanies Lynch syndrome:

"I would feel worried for them. Knowing what I go through every 18 months and what I have to do. If they could handle the same thoughts that go through my own head. Because there's a different kinds of thoughts that go through your head when you know you're a carrier and you don't know how other people would react." (High health anxiety male)

"[What bothers me most] is just to know that they have to live with that constant wonder if they're going to get cancer or not." (High health anxiety female)

"I feel bad that this is going to weigh a lot on them. I don't know how much on either one because they're so different but it - that's going to be a lot if I tell them in their late teens or in their twenties, if I felt devastated when I was in my thirties, I can't imagine how they're going to feel when they're in their twenties. Your twenties are meant to be free 
and fun and explore the world and not have any worries right?" (High health anxiety female)

Cancer screening/prophylactic procedures. Both the high and low health anxious parents discussed similar concerns with regards to children undergoing cancer screening and prophylactic procedures. The focus of these concerns was on having to undergo regular cancer screenings and on the decision-making and surgery involved in prophylactic procedures (e.g., prophylactic hysterectomy):

"[I worry most about] them having to live with this. Having colonoscopies from 21 on, and having to worry about cervical and ovarian cancer and whether that will rush them into having families. That's a long life of being concerned about things you shouldn't have to worry about at 21." (High health anxiety female)

"Well my aunt also has [Lynch syndrome] and she had to go through a hysterectomy and like she's older. She's 60 but had to go through a hysterectomy and a fair bit of surgery and pain. I guess with the girls that might be the worst... that they'd have to go through that. Maybe the chance they'd have to go through that if they tested positive [for Lynch syndrome] and I guess that would be the worst part." (Low health anxiety male)

Cancer. Not surprisingly, the most commonly feared consequence of Lynch syndrome

that parents in both the high and low health anxiety groups reported was that their children would develop cancer. Interestingly, parents specifically mentioned that they were less concerned about their children having colon cancer, but were more focused on cancers that were more difficult to detect and treat:

"I guess I worry about the forms of cancer that he could develop that are more serious. I view bowel cancer as the one cancer that's more easily remedied, so to speak. You know if he were to develop leukemia or bone cancer, those don't always have such a great outlook. So I guess when I worry about him it's if he ever becomes diagnosed, what kind of cancer would he be diagnosed with. That's my worry." (High health anxiety female)

"With [my daughter] being a female in the family and based on family history, there's a - it's more different areas of cancer that have affected the females. There's a higher rate of ovarian cancer, there's higher rates of bowel cancer of course, higher rates of brain 
tumours. So it's just a little bit of a concern, how to test for all these things, you know?" (High health anxiety male)

"I guess my main worry is that they have stage 4 cancer and can't do much about it." (Low health anxiety male)

Coping Strategies. Parents were asked to report on the behaviours or strategies they engage in as a method of managing their anxiety about their child's health. These coping strategies were grouped into three main categories: 1) Solution Focused Coping (e.g., becoming informed about Lynch syndrome, making healthy lifestyle changes, seeking instrumental support), 2) Emotional Support and Acceptance Based Coping (e.g., seeking emotional support from loved ones, positive reappraisal of the situation, acceptance of the situation), and 3) Dysfunctional Coping (e.g. denial, avoidance, rumination, self-blame). Differences emerged between the high and the low health anxiety groups with regards to the type of coping strategies they used to manage their worries about their children's health. The high health anxiety group discussed using solution focused coping strategies most often, whereas the low health anxiety group more frequently utilized using emotional support and acceptance based coping strategies. Dysfunctional coping strategies were rarely reported, however, only the high health anxiety group indicated using dysfunctional coping strategies.

Solution focused coping. With regards to solution focused coping strategies, although the high health anxiety group used this style of coping most frequently, both the high and the low anxiety parents reported similar examples. In particular, both groups discussed solution focused coping strategies such as helping their children to live a healthy lifestyle, educating them about Lynch syndrome, and the actions they can take to prevent cancer:

Solution focused coping: Healthy lifestyle for children 
"I spend a lot of time trying to talk to [my children] about eating healthy, and how important that is, and why it's important to eat healthy foods, and how that can prevent you from getting cancer. I probably talk to them about cancer more than the average person does, but not in a-yeah not in a way like "you're going to get cancer", just like these are good foods for helping to fight cancer." (High health anxiety female)

"I just feel like I need to put [my children] on a good path. Not that my parents didn't because they didn't know any other back then but I think - not that I want to scare my children - just if I put them on a better path there's more hope for them to lead a healthier lifestyle when they're older. That it won't be such a-you know, if we're eating junk food and we're going to McDonald's every week or whatever - I feel like that is a good start for them, a good path. Because they can't - it doesn't even matter whether or not they have Lynch - they can't have that lifestyle. And if they do have Lynch, then they really have to be careful. At least that's my perspective." (High health anxiety female)

"And I don't even think we'll worry about [the kids having Lynch syndrome] so much in that we'll find out what things we can do and should do and make sure that we help our kids with that. With a diagnosis, with healthy living, with counselling, whatever they need. But we're just not to that point yet. But I'm prepared for that point, to do the things that need to be done." (Low health anxiety male)

Solution focused coping: Educating children about Lynch syndrome

"I have to look at it like now it's my job to put them on a good path. To teach them, to educate them, that [Lynch syndrome] may be in your life so you now have to take responsibility. It's a lot... Well I certainly would be, I would hope that I'd be around in order to kind of guide them, or discuss about what's been in the family. But also to educate. I certainly hope that I would be very much involved in the conversation of what my daughter and my son would need to do." (High health anxiety female)

"Staying on top of your health is paramount and journaling [about cancer and treatments] because it is surprising how much you forget. Having that journal for them in the future might help them see similarities or go to the doctor to get certain things checked. Definitely reading as much as you can about the risk factors and food - which is something you can control. Also bringing your doctor up to speed and staying on top of appointments helps." (High health anxiety female)

"I stay calm and try to talk to them about it. They were little when I had [cancer]. They know what I go through and what they would have to do to make sure they don't let it get away from them. I just tell them it's something that's part of their life now that they have to look after." (Low health anxiety male) 
Emotional support and acceptance based coping. Both the high and low health anxiety parents commonly reported emotional support and acceptance based coping strategies, although the low anxiety group reported using these strategies more frequently. Notably, in contrast to the varying examples of emotional support and acceptance based coping strategies participants reported using when they thought about their own health (e.g., emotional support, acceptance, positive reappraisal), only one coping strategy was noted when discussing coping with their worries about their children. Specifically, the only example provided by parents was utilizing an acceptance based stance regarding their children having Lynch syndrome:

"It's too bad [that they may have Lynch syndrome], but that's just the way life is. People, some people have the history of heart disease in their family so it's passed on from generation to generation. So I don't know, I don't think sons blame their fathers for passing on heart disease it's just the way it is. There's no heart disease in our family, it's cancer. Well I can't blame my father and he can't blame his father it's just something they got in their genes and it's passed down." (Low health anxiety male)

"And since I couldn't control [whether or not they have Lynch syndrome], what could I have done? I couldn't have done anything different to protect my children. It's just the human - I want to say condition- but it's just people procreate. It's just the way people come out." (Low health anxiety male)

"What you've got to understand too, is that it is what it is... Regardless of whether you got it or not, well what the hell are we going to do about it, right”? (High health anxiety male)

Dysfunctional coping. Dysfunctional coping strategies were only mentioned by three individuals; all of whom were in the high health anxiety group. No items were coded as dysfunctional coping for the low health anxiety group. For the high health anxiety individuals, catastrophizing about their children's health and avoidance were the dysfunctional coping strategies parents endorsed: 
"I try to make connections between what I see in [my child's] health and mine. That gives me a window into what they have or they don't have. My oldest is more like my husband but I can't let that fool me... Every new symptom that comes up is like a tick that confirms that she has [Lynch syndrome." (High health anxiety female)

"I'm not letting myself think that far ahead about my kids yet. I'm thinking right now not about my kids as adults who are worried about this yet... I think I intentionally don't let myself think that far ahead." (High health anxiety female)

\section{Discussion}

The purpose of the present study was to examine the impact of health anxiety on parents

with Lynch syndrome. Specifically, the study was designed to contrast the experiences of parents with Lynch syndrome with high health anxiety to those with low health anxiety. Differences in the two subgroups' experiences were compared with regards to their perceptions of their own health, their thoughts and emotions of potentially passing down the genetic mutation to their children, and their perceptions of their children's health.

\section{Overall Similarities and Differences between High and Low Health Anxious Patients}

In examining the interview responses as a whole, a number of patterns emerged with regards to the overall similarities and differences between the individuals in the high and low health anxiety groups. Overall, it cannot be denied that the experience of having Lynch syndrome is stressful for individuals. It appears that this stress creates a common experience for the vast majority of parents with Lynch syndrome. In particular, the majority of the commonalities between the high and low health anxiety groups appear to be with regards to their perceived benefits of having knowledge about Lynch syndrome (e.g., increased access to medical professionals, cancer surveillance procedures, etc.). However, individual differences seem to arise with regards to their perceptions of the negative experiences or consequences that are associated with Lynch syndrome. For example, the high health anxiety group reported 
engaging in dysfunctional coping strategies, they spoke more frequently about the negative consequences of receiving genetic testing, and they discussed the negative emotional and psychological consequences of Lynch syndrome. It is evident, that for individuals with high health anxiety, the negative aspects of Lynch syndrome are much more salient and relevant than for individuals who experience lower levels of health anxiety.

However, contrary to what was anticipated, more similarities than differences were observed between the high and low health anxious patients with regards to their perceptions of passing down the Lynch syndrome gene to their children and their perceptions of their children's health. Within these categories, it was evident that the low health anxious individuals worry more about their children's health than they do their own, whereas the high health anxiety patients tend to worry about their children's health slightly less than they worry about themselves.

Perhaps the biggest overall difference between the high and low health anxious patients was with regards to their perceptions about themselves. As expected, the high health anxiety individuals worried significantly more about their health, demonstrated a hypervigilance towards physical symptoms, and spoke of their worries in a more extreme manner than the low health anxiety patients.

\section{The Impact of Health Anxiety on Perceptions of Self}

Consistent with both the HA model and previous literature on health anxiety, individuals with high health anxiety worried a great deal about their health and this anxiety translated into a hypervigilance towards bodily symptoms they believed could be indicative of a health problem. In contrast, individuals with low levels of health anxiety worried infrequently about their health and their anxiety was best represented as an increased awareness of their bodies and their risk of 
developing cancer (as opposed to worries). Given that health anxiety is defined as a preoccupation with the fear that one has, or may develop, an illness (American Psychiatric Association, 2013), these differences in the level of worry about personal health between the two groups were certainly expected. Our findings are consistent with the HA model and previous research underscoring that health anxiety increases one's attention to illness information, which can manifest as hypervigilance towards signs of illness (Owens, Asmundson, Hadjistavropoulos, \& Owens, 2004; Salkovskis \& Warwick, 1986).

One surprising finding, however, was that no differences emerged between the descriptions high and low health anxious individuals gave about their current health. Previous research has demonstrated that individuals with high health anxiety have a constricted definition of what constitutes good health, such that they tend to believe that good health is completely symptom-free (Barsky, Coevtaux, Sarnie, \& Cleary, 1993; Weck, Neng, Richtberg \& Ulrich, 2012). As such, we expected individuals with high health anxiety to evaluate their health more negatively than those with low health anxiety. However, despite the knowledge of their Lynch syndrome carrier status, nearly all patients stated that they were in overall good health. It is also of note that participants very rarely discussed their diagnosis of Lynch syndrome when asked to describe their perceptions of their current health. Such findings suggest that Lynch syndrome is not viewed as a current impediment to one's health, but may simply represent a potential threat for future health. This is an important and novel finding that has yet to be reported in the existing literature.

Although both the high and low health anxious individuals expressed similar concerns about their futures, qualitative differences between the groups emerged with regards to the severity of their worries, with more extreme and severe worries being reported by individuals 
with high health anxiety. The primary concerns discussed by all participants, regardless of their degree of health anxiety, were fears of developing or experiencing a recurrence of cancer, dying from cancer, and the impact that cancer or death would cause to their family and loved ones. Similar apprehensions have been noted in previous literature, with concerns for one's future health, death, and worries about other family members frequently reported by individuals who have received a diagnosis of Lynch syndrome (Aktan-Collan et al., 2013; Carlsson \& Nilbert, 2007; Koehly et al., 2003; Meiser, 2005; van Oostrom et al., 2007; Wilson et al., 2004). In examining patients' worries in greater detail, however, clear group differences emerged with regards to participants' descriptions of their feared consequences of having Lynch syndrome, with the high health anxious individuals speaking more frequently about their worries and describing their worries as more extreme (e.g., discussed dying from cancer as inevitable). Such group differences were expected given prior research showing catastrophic thinking patterns in those with high health anxiety (Hadjistavropoulos, Hadjistavropoulos, \& Quine, 2000; Hitchcock \& Mathews, 1992; Marcus, Hughes, \& Amau, 2008; Rief, Hiller, \& Margraf, 1998) and a propensity to overestimate the frequency and likelihood of serious illnesses (Haenen, de Jong, Schmidt, Stevens, \& Visser, 2000; Marcus, 1999; Marcus \& Church, 2003). Interestingly, individuals with high health anxiety also demonstrated a tendency to focus on the emotional consequences their family members would experience if they died from cancer, whereas those with low health anxiety spoke about their practical concerns (e.g., finances). This finding underscores some of the cognitive differences observed in the patients with high and low health anxiety. As our findings indicate, individuals with low health anxiety experience less distress when thinking about their health and future and, as such, they demonstrate a tendency to think quite pragmatically when asked to discuss the consequences of having cancer or dying. In 
contrast, patients with high health anxiety are acutely aware of the emotional distress they experience with regards to their Lynch syndrome and have difficulties managing these emotions. It appears likely that these individuals are projecting that their loved ones will have similar negative emotional reactions and also struggle to cope should a cancer diagnosis or death occur. This would account for the worry high health anxious patients experience about the emotional impact Lynch syndrome will have on loved ones.

In line with this, clear differences emerged between the high and low health anxiety groups with regards to their use of dysfunctional coping strategies to manage their health related worries. While the low health anxiety group did not report using any dysfunctional coping strategies, nearly every high health anxiety participant reported at least one type of dysfunctional coping strategy (e.g., catastrophizing, avoidance, rumination). Previous research has noted that individuals who have undergone testing for Lynch syndrome utilize a wide range of coping strategies to manage the stress of living with the knowledge of hereditary cancer. These include actively suppressing emotions (e.g., denial, projecting negative feelings on others, distraction, and avoidance), mentally preparing themselves for future health related outcomes, telling themselves that cancer can be overcome, and using acceptance-based strategies (Carlsson \& Nilbert, 2007). However, no prior research has examined if these coping strategies vary as a result of Lynch syndrome carriers' levels of health anxiety. Findings from the broader health anxiety literature, however, provide evidence that supports the relationship between varying levels of health anxiety and functional versus dysfunctional coping strategies. For example, Hadjistavropoulos and colleagues (1998) found that individuals with higher health anxiety engaged in fewer protective coping strategies (e.g., using objectivity when monitoring their physical symptoms) when dealing with pain than non-health anxious individuals and 
hypothesized that health anxious individuals may have a deficiency in protective coping behaviours. These findings differ somewhat from ours, as we did not find that health anxious individuals utilized less positive coping strategies, but rather engaged in more negative coping strategies. However, these findings support the notion that health anxiety influences the coping strategies individuals engage in when anxious about their health. An important finding from Study 2 is that dysfunctional coping appears to be unique to high health anxious Lynch syndrome patients. This finding highlights a possible target for future interventions with this population.

\section{The Impact of Health Anxiety on Perceptions of Passing Down Lynch Syndrome to Child}

Given that individuals experiencing high levels of health anxiety have a tendency to overestimate both the probability and the severity of illness (Warwick \& Salkovskis, 1990), it was predicted that parents - when thinking about the possibility of their child being predisposed to developing cancer - would experience heightened negative emotional reactions and increased worry about their children having Lynch syndrome. This expectation was based on prior research showing that parents experience significant worry about their children being Lynch syndrome carriers (Bartuma et al., 2012; Carlsson \& Nilbert, 2007; Gaff et al., 2006; Koehly et al., 2003; Meiser, 2005; Wilson et al., 2004). However, it appears that one's own health anxiety does not influence the degree to which individuals worry about their children's health. Our findings indicate that the exacerbated worry health anxious individuals experience is specific to their personal health and does not necessarily generalize to their children.

One possible explanation for this finding relates to the age of the children of the participants in our study. Statistically, the average age that an individual with Lynch syndrome develops cancer is 46 years old (De Jong et al., 2004; Hampel et al., 2008). Within our sample, 
$100 \%$ of the children that parents discussed were under age 46 and $94 \%$ of the children were under age 40. Given that the children in the current sample are under the age when Lynch syndrome associated cancers typically develop, it may be the case that parents are not overly anxious simply because they believe their children are too young to be affected. An additional explanation as to why parental health anxiety does not generalize to their worries about their children's health relates to the detection of physical symptoms. According to the HA model (Warwick \& Salkovskis, 1990), catastrophic misinterpretations of benign physical sensations is a key component of elevated health anxiety. Because parents are not able to attend to the physical symptoms that occur in their children's bodies to the same degree that they can detect minute physical changes in themselves, it is possible that the cognitive and behavioural cycle associated with increasing one's health anxiety is not triggered and, as such, health anxiety related to one's child is significantly minimized.

With respect to emotions, parents in both groups reported experiencing feelings such as guilt, sadness, anxiety and anger. These emotional reactions are consistent with previous research which has noted that parents undergoing genetic testing for cancer mutations frequently report feelings of guilt, depression, and anxiety when thinking about the possibility of having passed down a genetic mutation to their child (Bartuma, Nilbert, \& Carlsson, 2012; Carlsson \& Nilbert, 2007; Murakami et al., 2004; Iris Van Oostrom et al., 2006). Moreover, specific to Lynch syndrome, parents report experiencing negative emotions about passing down the genetic mutation to children despite having an awareness that they have no control over whether their child is a carrier (Esplen et al., 2011). Although it is evident that parents experience a range of negative emotions when thinking of the possibility of passing down Lynch syndrome to their child, again, our data indicate that health anxiety does not impact the emotional experience of 
parents with regards to their feelings about passing down the condition to their children.

One possible explanation for the lack of differences in emotional responses of parents is that perhaps the emotions themselves do not differ as a result of one's level of health anxiety, but the intensity of said emotions does. As emotional intensity was not assessed in the present study, this cannot be directly commented on. However, research examining the affect of individuals with health anxiety has noted higher amounts of negative affectivity in individuals diagnosed with the previous DSM-IV-TR diagnosis of hypochondriasis (Haenen et al., 2000; Watson et al., 1988) and has also found that a moderate to large relationship exists between general levels of emotional distress and health anxiety (Marcus et al., 2008; Marcus \& Church, 2003). Therefore, it is possible that differences between the two subgroups would have emerged if the strength of the emotions were examined.

When considering the positive outcomes of genetic testing for Lynch syndrome, consistent with predictions, no differences were observed between high and low health anxious patients. Previous literature has found that parents consistently cite the desire to obtain cancer risk information for their children as the primary factor that motivated them to seek genetic testing (Claes et al., 2004; Esplen et al., 2001; Lerman et al., 1998; Patenaude et al., 2006). Parents are adamant about the importance of gaining knowledge about cancer risk and available options, as increased knowledge generates a sense of mastery and control over a stressful situation (Maloney, Edgerson, Robson, Offit, Brown, Bylund, \& Kissane, 2012). Additionally, patients who have opted for genetic testing of Lynch syndrome report that factors such as being able to detect cancer early, reducing their uncertainty, and gaining information as to whether undergoing screening procedures is necessary are the primary benefits of genetic testing (Claes et al., 2004; Esplen et al., 2001; Hadley et al, 2003). Results from our study indicated that 
parents reported similar, although slightly varied, benefits of having their children undergo genetic testing. For example, the benefits of having access to regular cancer screenings, medical professionals, and research appears to contribute to parental beliefs that genetic testing can benefit their children from a medical standpoint. Our findings indicate that regardless of health anxiety levels, parents are able to identify and appreciate a number of the positive outcomes associated with genetic testing for Lynch syndrome.

What differed between the high and low anxious patients, however, were their perceptions of the negative consequences of genetic testing. In particular, parents with high health anxiety described negative emotional and practical consequences related to testing; expressing concern over their children's ability to manage the emotions that come with a diagnosis of Lynch syndrome and worrying about their children being able to obtain insurance in the future. Such fears about emotional well-being and the impact of genetic knowledge on one's future (e.g., obtaining life insurance, a mortgage, employment, etc.) are certainly not novel findings. Similar concerns have been reported by individuals undergoing an array of genetic testing (Apse, Biesecker, Giardiello, Fuller, Bernhardt, 2004; Carlsson \& Nilbert, 2007; McInemey Leo et al., 2005; Norum, 2000; Rodriguez-Bigas, Vasen, O’Malley, Rosenblatt, Farrell, Weber, Petrelli, 1998). However, our research highlights that differences in the perceptions of negative consequences of Lynch syndrome are dissimilar between parents with high and low health anxiety. Although the degree to which parents are anxious about their children's health is comparable between individuals with high and low health anxiety, the content about what parents are thinking and worrying about differs as result of parental health anxiety levels. This finding is likely a reflection of the distorted cognitions of individuals with high health anxiety; with these parents engaging in catastrophic thinking, asking future-based 
'what-if' questions, and fortune-telling.

\section{The Impact of Health Anxiety on Perceptions of Children's Health}

Similar to the findings from parents' reports of their experience of potentially passing down Lynch syndrome to children, health anxiety appeared to play only a minimal role in impacting the perceptions parents held about their children's health. Parents in both groups generally reported that their children were healthy and did not discuss Lynch syndrome when evaluating their children's health. Interestingly, the way in which parents described their child's health was nearly identical to the way in which parents talked about their own health. That is, they did not mention Lynch syndrome in their description of their health, tended to report that they were in good health, and only reported negative health if it was related to discrete, current health problems. Although individuals with high health anxiety have been shown to exhibit a constricted view of what constitutes "good health" (Barsky, Coevtaux, Sarnie, \& Cleary, 1993; Weck, Neng, Richtberg \& Ulrich, 2012), our findings indicate that Lynch syndrome is not viewed as a factor influencing whether one views themselves or their child as healthy versus not healthy. Similar to their own personal assessments of health, parents do not appear to conceptualize Lynch syndrome or the potential of having Lynch syndrome as a defect in their children's health. Instead, Lynch syndrome appears to represent a vulnerability or a potential future threat to their child's health. This is understandable considering that the majority of children with the Lynch syndrome gene are asymptomatic. Moreover, individuals typically utilize heuristics based on their family histories with cancer to guide expectations about one's vulnerability to developing cancer (Kenen et al., 2006; Werner-Lin, 2007). Consequently, if the majority of individuals in a family have been healthy and cancer-free until much later in adulthood, this history is likely to play a large role in parents viewing their children as healthy 
individuals.

Similarly, regardless of their level of health anxiety, parents exhibited a moderate level of worry about their children's health. Although Warwick and Salkovskis' (1990) HA model is focused on factors that trigger an individual's anxiety about their own health (e.g., catastrophic misinterpretations of benign physical sensations, overestimation of the probability of serious illness, etc.), we believed that it would be likely that parents with high health anxiety would experience a similar process of hypervigilance and distorted cognitions in regards to their children's physical symptoms and health. However, our findings do not support this conclusion. Findings from the genetic cancer literature report that patients commonly experience considerable preoccupation and worry about their child's risk for hereditary cancers (Clarke, Butler, \& Esplen, 2008; MacDonald et al. 2010; Miesfeldt et al. 2003; Peshkin et al. 2010; Tercyak et al. 2001, 2002, 2007), however, prior research has not examined the relationship between health anxiety and worry about one's child's health. It appears that worry about children's health is a shared experience that exists among most parents with genetic predispositions to cancer, regardless of their personal health anxiety levels. The fact that parental health anxiety does not appear to influence the degree to which parents worry about their children's health is a novel finding.

The primary worries parents experience when thinking about their child having Lynch syndrome are about the psychological consequences their child would experience, the cancer screenings and prophylactic procedures they may have to undergo, and their child being diagnosed with cancer. Similar concerns have been reported in the genetic cancer literature (e.g., Lynch et al., 2006), however, our study extended these findings by noting that parent worries tended to be focused on specific forms of cancer. Parents were particularly worried that that the 
type of cancer would be a form that would be difficult to detect (e.g., ovarian cancer as opposed to colon cancer). Such fears are overall quite realistic given that surveillance for endometrial and ovarian cancers are less effective than screening for colorectal cancers (Vasen et al., 2013) and indicate that individuals with Lynch syndrome have a good understanding of the risks, surveillance options and outcomes of cancers related to Lynch syndrome.

One clear difference that emerged was that only the patients with high health anxiety reported worries about the negative psychological consequences their children would endure if diagnosed with Lynch syndrome. This pattern is similar to what individuals with high health anxiety reported about their own feared consequences of having Lynch syndrome. Previous research has noted a variety of fears that patients experience when considering undergoing genetic testing, including undergoing medical procedures, being ill, managing the associated anxiety and uncertainty, and dying from cancer (Aktan-Collan et al., 2013; Carlsson \& Nilbert, 2007; Koehly et al., 2003; Meiser, 2005; van Oostrom et al., 2007; Wilson et al., 2004). Our research extends this work by demonstrating that individuals with high health anxiety are more inclined to focus on the emotional experience of Lynch syndrome and assume that their children will have a similar experience to theirs.

Additionally, it appears that health anxiety influences the type of coping strategies parents use to manage their worry about their children's health. Parents with low health anxiety utilized solution focused coping strategies (e.g., helping their children to live a healthy lifestyle, educating them about Lynch syndrome) most often, whereas parents with high health anxiety tended to use emotional support and acceptance based coping (e.g., utilizing an acceptance base stance regarding their children having Lynch syndrome). This is interesting because it differs somewhat from the types of coping strategies patients endorsed using to cope with their anxiety 
about their personal health. Parents with low health anxiety consistently utilize solution focused coping strategies, regardless of if they are anxious about their own health or their children's health. However, parents with high health anxiety reported a tendency to use solution focused coping in response to their own health anxiety, but emotional support and acceptance based coping strategies to cope with their worries about their children's health. This finding may be a reflection of the parent's perceptions of their child's health and future. Folkman and Lazarus (1990) suggest that both emotion and solution focused coping are commonly utilized in stressful situations, however, the relative effectiveness of each coping strategy at reducing distress varies depending on whether a situation is viewed as controllable or uncontrollable; with solution focused coping being most effective in controllable situations and emotion focused coping being helpful in uncontrollable situations. In our study, it is possible that parents with low health anxiety view their children's health as controllable and therefore engage in strategies focused on promoting health or preventing disease (i.e., solution focused coping), whereas parents with high health anxiety feel as if little can be done to change their child's future health condition and they therefore engage in emotion focused strategies.

Although the coping strategies utilized to manage concerns about one's child having Lynch syndrome have not been formally examined in previous literature, research examining coping strategy use by mothers whose children had been recently diagnosed with cancer have found an increased use of emotion focused coping strategies compared to mothers whose child had been diagnosed with an acute health condition (Barerra, D'Agostino, Gibson, Gilbert, Weksberg, \& Malkin, 2004). Interestingly, in the aforementioned study, increased use of emotion focused coping was associated with higher levels of depression but not anxiety. Findings from the broader medical literature, however, have noted a link between greater use of 
emotional preoccupation coping and higher health anxiety (Hadjistavropoulos et al., 2012;

Kehler \& Hadjistavropoulos, 2009). In line with this, our findings indicate that for parents with Lynch syndrome, greater use of emotion based coping is associated with higher levels of anxiety.

Furthermore, only parents with high health anxiety reported using dysfunctional coping strategies (e.g., catastrophizing, avoidance) when worrying about their children's health. This is similar to the coping strategies reported for dealing with worries about one's own health, with only the high health anxiety group reporting dysfunctional coping strategies. Previous research has noted that individuals with Lynch syndrome endorse utilizing dysfunctional coping strategies (e.g., denial, projecting negative feelings on others, distraction, and avoidance) when attempting to manage Lynch syndrome related anxiety (Carlsson \& Nilbert, 2007). However, the present findings are novel in that they highlight that health anxiety influences the ways in which parents cope with worries related to their children's health, with parents with low health anxiety primarily utilizing solution focused coping and those with high health anxiety using emotional support and acceptance based coping. Moreover, the present research also demonstrates a link between dysfunctional coping and anxiety about one's children's' health.

\section{Limitations of the Present Study}

It is important to acknowledge the limitations present within the current study. Specifically, with regards to the sample of individuals selected to participate in the interviews, demographic variables related to their children were not controlled for. As such, the ages of children ranged from infants to grown adults. This age range is particularly important as current guidelines indicate that children should typically not be screened for cancer susceptibility until

they are 18 years of age or older. Consequently, when parents were asked to reflect on how they felt about their child's Lynch syndrome carrier status and the genetic screening process, parents 
with younger children could not report on their actual feelings and reactions but had to reflect on how they imagine they would feel or react. Similarly, numerous children over the age of 18 had not yet undergone genetic testing for Lynch syndrome. In these instances, parents were also asked to imagine their reactions as opposed to reporting on the actual experience retrospectively. A second limitation relates to the study design. The current study was designed to compare individuals with the highest and lowest levels of health anxiety so that their experiences of being a Lynch syndrome carrier could be examined. Although such purposive sampling is a commonly used methodology to examine group differences, it is possible that by not sampling the full continuum of individuals with health anxiety, important differences in the experience of being a parent with Lynch syndrome were missed. For example, it is possible that individuals falling within a mid-level of health anxiety have a unique experience. While our data do not give any indication that this is the case, as the full spectrum of individual health anxiety levels was not qualitatively examined we cannot definitively comment on this. An additional concern related to our sample is the use of the FGICR registry for patient recruitment. The FGICR registry was utilized as a method to confirm that all participants had a diagnosis of Lynch syndrome. However, it is possible that people who carry the Lynch syndrome genetic mutation but have never been tested (and are therefore not include in the FGICR registry) have a different form or different levels of anxiety all together.

One concern regarding the sample of participants in the present study is that the homogeneity of the individuals interviewed may limit the generalizability of our findings. In particular, the vast majority of the sample were Caucasian, employed, highly educated and in a romantic relationship. Individuals with different backgrounds and demographics might yield somewhat different responses to the interview questions that were posed. 
A final issue to consider is the use of qualitative content analysis to examine the differences between individuals with high and low levels of health anxiety. Content analysis was selected as it allowed for individual experience, knowledge, and insights about Lynch syndrome to be examined. The present research utilized a theoretical model to guide the interview questions and the interpretation of the data. However, should we have selected to use a different epistemological framework to view the data, this would have altered the way in which data would have been collected, analyzed, and interpreted. Because participants were not directly asked what services or supports they would find helpful, this information did not emerge in the data. However, if a social constructive lens or a phenomenological lens had been used, these approaches could have been more informative in developing a greater understanding of what forms of services parents with Lynch syndrome need. This could have been particularly helpful in aiding clinical extrapolation to devise a future intervention for these patients. 


\section{DISCUSSION AND INTERGRATION OF FINDINGS FROM STUDIES I AND II}

The overarching aims of this research were, among those with Lynch syndrome, to identify the severity, predictors, and consequences of health anxiety as well as to examine the impact of health anxiety on parents with Lynch syndrome. Important differences between the cognitions and behaviours of high and low health anxious individuals were illuminated in both Study I and Study II. Overall, the combination of quantitative and qualitative methodology in the present research allowed for a more robust analysis of the construct of health anxiety among individuals with Lynch syndrome. Interpreting findings regarding anxiety and general psychological distress in medical populations is challenging as a certain degree of anxiety and distress are to be expected when individuals face significant health threats. As such, researchers can often run the risk of over-pathologizing a normative psychological phenomenon. The use of quantitative and qualitative methodology in our research is crucial as it aids in evaluating the challenging question: 'To what extent is health anxiety maladaptive among Lynch syndrome carriers?'

Quantitative results from Study I of the present research revealed that health anxiety is a relevant concern for individuals with Lynch syndrome, with $30 \%$ of the sample reporting levels that were clinically significant. Although this percentage is substantially higher than the estimated three to six percent of the general population that experiences high health anxiety (Bleichhardt \& Hiller, 2007; Sunderland et al., 2012), the percentage of our sample with significant health anxiety is consistent with the rates of high health anxiety demonstrated in medical populations (e.g., Grassi et al., 2004; Janzen \& Hadjistavropoulos, 2008; Kehler \& Hedjistavropoulos, 2009; Stark et al., 2004). Looking exclusively at the findings from Study I, it 
may be tempting to conclude that elevated health anxiety is simply a byproduct of medical illnesses that will affect approximately one third of individuals with medical conditions.

The consistent rate of high health anxiety among diverse medical populations begs the question; 'Are we over-pathologizing health anxiety among individuals with genetic predispositions to cancer?' If anxiety is normative and stable over time among medical populations, should clinicians simply validate the anxiety and regard it as an expected consequence of illness? The difficulty that exists for researchers and clinicians working with populations at an increased risk of developing cancer is in determining if and when health anxiety extends beyond the realm of an appropriate level of vigilance related to cancer monitoring and into a pathological level of anxiety.

The challenges present in distinguishing between normative anxiety and maladaptive anxiety among cancer populations are numerous. According to commonly used psychiatric diagnostics, anxiety becomes pathological when the level of fear is disproportionate to the level of threat, the symptoms persist or worsen without intervention, and the symptom severity is distressing and causes a disruption in normative functioning (American Psychiatric Association, 2013; World Health Organization, 1992). As highlighted by Stark and House (2000), however, it is difficult to judge when anxiety is disproportionate to the threat of cancer. Moreover, the duration and intensity of anxiety symptoms can be challenging to establish because of the labile and situationally-bound nature of anxiety. Consequently, focusing on the degree of impairment caused by anxiety related cognitions (e.g., intrusive thoughts about disease, death, or disability causing disruption in social functioning, concentration, sleep, etc.) and behaviours (e.g., avoidance, reassurance seeking, and repetitive physical checking interfering with day-to-day functioning) can be useful. Our quantitative data provided some information about possible 
impairment - showing increased health anxiety to be associated with greater medical utilization to gastroenterologists and emergency departments - but other dysfunctional thoughts and behaviours were not assessed in Study I.

However, the qualitative findings from Study II do elucidate clinically relevant differences between individuals experiencing high versus low health anxiety and aid in the evaluation of normative versus pathological health anxiety. Clear differences emerged with regards to the cognitions of the individuals highest and lowest in health anxiety. In addition to worrying more about their personal health, those with high health anxiety frequently reported distorted cognitions such as catastophizing (e.g., "When my body is feeling a little different... my mind always goes to cancer.") and fortune telling (e.g., "In my mind, I will die of cancer. I will get cancer again."). From a behavioural perspective, high health anxious participants also demonstrated a hypervigilance towards physical symptoms and sensations and engaged in dysfunctional coping strategies. Overall, individuals with high health anxiety are much more focused on the negative aspects or consequences of Lynch syndrome and view the disorder as more impairing in their day-to-day life; factors which are indicative of the clinical significance of health anxiety among this population.

Not only is health anxiety important to target to reduce distress for the individual, but research also suggests that untreated health anxiety can have an intergenerational influence; negatively impacting future generations. Within our research, relatively few differences were observed between how the high and low health anxious parents viewed their children's health. However, previous research has noted correlations between parental health anxiety and children's beliefs about health and health related behaviours; suggesting that health anxiety is a learned phenomenon and is passed down to the next generation (Köteles, Freyler, Kökönyei, \& 
Bárdos, 2014; Lau, Quadrel \& Hartman, 1990; Salkovskis \& Warwick, 2001; Taylor, Jang, Stein, \& Asumndson, 2008; Taylor, Thordarson, Jang, \& Asmundson, 2006). Our data cannot comment directly on the intergenerational transmission of health anxiety as children were not interviewed. However, the findings from previous research provide additional support to the idea that elevated health anxiety among parents with Lynch syndrome should be targeted to prevent future generations from experiencing similar distress. This is particularly important in genetic conditions such as Lynch syndrome because of the likelihood that children will experience similar health experiences and challenges to their parents. Adequately addressing parental health anxiety could prevent future generations of Lynch syndrome carriers from experiencing their own elevated health related anxiety and distress.

Overall, due to the increased risk of cancer patients with Lynch syndrome face, it is certainly expected and even beneficial for patients to exhibit a heightened awareness and some anxiety regarding their physical health. In fact, it is important that patients learn to monitor their bodies for physical changes, interpret their symptoms, and seek medical attention when warranted. While a degree of health anxiety is likely adaptive, clinically elevated health anxiety becomes problematic when it leads individuals to overestimate the probability and seriousness of illness, become preoccupied with a suspected illness, constantly monitor their bodies and interpret any and all physical symptoms as threatening, and seek medical attention and reassurance to the extent that it interferes with psychosocial functioning (Abramowitz, Olatunji \& Deacon, 2007; Owens et al., 2004; Stark \& House, 2000). In line with this, the findings from our research demonstrate that the thinking styles and behaviours exhibited from those experiencing high health anxiety are distinct from those who experience low health anxiety and warrant further clinical attention and potential intervention. 


\section{Clinical Implications}

Our data have important clinical implications for the management of health anxiety in individuals with Lynch syndrome. Clinicians working with this population should be aware that health anxiety is elevated, with $30 \%$ of individuals reporting health anxiety within the clinical range. Patients with Lynch syndrome who are younger, have higher rates of depression, anxiety, and worry interference, and demonstrate a tendency to use emotional preoccupation as a coping strategy have higher health anxiety. Identification of these variables in individuals with Lynch syndrome could help to highlight the patients who are at a greater likelihood of requiring interventions for elevated health anxiety. Moreover, as our research demonstrates, individuals with high health anxiety are more likely to catastrophize physical symptoms, predict negative health outcomes, experience heightened psychological distress, engage in problematic coping strategies, and use more medical services. These findings, taken together with previous research which has clearly demonstrated that health anxiety is associated with reduced quality of life (Grassi et al., 2004; Martin \& Jacobi, 2006), increased disability (Grassi et al., 2004), and increased medical resource use (Barsky et al., 2001; Martin \& Jacobi, 2006; Stark et al., 2004), highlight the necessity of finding helpful strategies to effectively manage health anxiety in individuals with Lynch syndrome.

The most widely studied and effective treatment for health anxiety is cognitive behaviour therapy (CBT). Specific forms of CBT for health anxiety have been developed based on Warwick and Salkovskis (1990) cognitive model of health anxiety. CBT for health anxiety involves a) education on the cognitive, behavioural, affective, and physiological components of worry about illness, b) helping individuals to challenge distorted negative thoughts and develop more realistic thoughts, c) decreasing reassurance seeking behaviours, d) decreasing avoidance 
of illness-related worries and e) exposure to illness-related worries (see Owens \& Antony; 2011; Taylor \& Asmundson, 2004; Tyrer, 2013). This therapy has been found to be effective in reducing health anxiety (Nakao et al., 2011; Sorensen et al., 2011; Warwick et al., 1996).

It is important to note that CBT for health anxiety specifically targets an individual's excessive worry about their personal health as opposed to their worry about the health of loved ones. This is appropriate for patients with Lynch syndrome, particularly given our finding that individuals with high health anxiety worry more about their own health than their children's health. Certainly it is clear that treatment should target individual health anxiety, however, given that both the high and low health anxious parents reported experiencing worry and anxiety about their children's health, the degree to which anxiety about children's health or negative emotions surrounding the passing of the Lynch syndrome gene to children should be targeted needs to be assessed on a case by case basis.

Components of CBT that appear to be particularly relevant for individuals with Lynch syndrome include helping patients to identify and challenge maladaptive negative thoughts and to develop adaptive coping strategies. It is important to note that CBT does not have to be administered exclusively by mental health professionals. Non-mental health specialists (e.g., nurses, primary care physicians) have been taught to provide effective CBT in a variety of settings (e.g., Chalder, McCrone, Darnley, Knapp, Jones, \& Wessely, 2006; Dopson, 2010; Foa, 2006). Moreover, components of CBT can be administered in a timely manner and could potentially be incorporated into regular medical visits. For example, during a medical appointment, it could be beneficial to help patients identify their worries or distressing thoughts, provide medically relevant information related to their fears, and help them to challenge their negative thoughts using cognitive restructuring techniques. 


\section{Future Directions}

As previously discussed, the present research contained certain methodological limitations that, if addressed, could bolster the current findings. Examples of methodological improvements include the use of a longitudinal design, obtaining a more demographically generalizable sample, and using alternative methods to self-report questionnaires.

From a conceptual standpoint, future research should focus on evaluating additional consequences of health anxiety among Lynch syndrome carriers. The current research examined the impact of health anxiety on overall medical service utilization; however, the validity of this self-report questionnaire used was limited. One potential option is to utilize health care records (e.g., OHIP records) as opposed to self-report and to control for medical comorbidity in analyses. Additionally, future research focusing exclusively on unnecessary medical visits could prove to be more informative than obtaining information on all medical appointments attended. Determining whether a medical visit is medically warranted or a form of reassurance seeking behaviour driven by health anxiety is challenging. Asking participants to report on the purpose of their visit or surveying medical professionals directly could help to distinguish between medically necessary and unnecessary visits.

Further, it is important that future research also examine additional consequences of health anxiety above and beyond medical service utilization. For example, examining the impact of health anxiety on factors such as quality of life, disability, day-to-day functioning, and familial relationships in Lynch syndrome patients could serve to highlight additional domains impacted by health anxiety. Moreover, it would be helpful to further investigate the type of reassurance seeking behaviours patients with Lynch syndrome engage in. The current study only examined medical utilization; however, it is likely that patients with Lynch syndrome seek 
reassurance from a variety of additional sources. These could include researching symptoms on the internet, engaging in body checking behaviours, or soliciting reassurance from loved ones.

Perhaps most importantly, it is imperative for future researchers working with individuals with Lynch syndrome to ask participants directly what services they desire or would view as potentially beneficial in helping to reduce their anxiety about their health. Although CBT has been proven to be effective at reducing health anxiety within medical populations, it is currently unknown the extent to which Lynch syndrome patients would be interested in psychological services. Perhaps receiving additional information, having increased access to medical professionals, connecting with other patients with Lynch syndrome via a support group, or providing parents with resources to aid in the communication of genetic risk information to children would be more in line with the services these individuals would like to receive. Assuming, however, that patients report interest in psychological services, it is necessary to determine the effectiveness of a CBT based intervention for participants with Lynch syndrome. Additionally, identifying the specific components of CBT (e.g., psychoeducation, cognitive restructuring, decreasing reassurance seeking behaviours, etc.) which are most effective in reducing health anxiety among this population would be helpful in guiding the development of a CBT-based intervention designed specifically for individuals with Lynch syndrome. 


\section{Appendix A}

\section{Study I Questionnaire Package}

Study ID

\section{Lynch Syndrome and Health Anxiety}

Today's Date:

1) Age:

2) With whom do you live? $\square$ Spouse/Partner $\square$ Self $\square$ Children $\square$ Other

3) Relationship Status: $\square$ Married/Partnered $\square$ Separated $\square$ Divorced $\square$ Widowed Single

Other

4) If you are in a relationship, how long have you been with your spouse/partner?

5) Do you have any biological children? Yes $\square \quad$ No $\square$

6) If you have any biological children, please complete the following table for each child:

\begin{tabular}{|l|l|l|l|l|}
\hline & $\begin{array}{c}\text { Current } \\
\text { Age }\end{array}$ & $\begin{array}{c}\text { Gender } \\
\text { (male/female) }\end{array}$ & Have they been tested for Lynch? & $\begin{array}{c}\text { If yes, are they a } \\
\text { lynch carrier? } \\
\text { (yes/no) }\end{array}$ \\
\hline Child 1 & & & Yes $\square \quad$ No $\square$ & \\
Child 2 & & & Yf yes, date tested: & \\
\hline & & If yes, date tested: & \\
\hline Child 3 & & & Yes $\square$ & \\
& & & If yes, date tested: & \\
\hline
\end{tabular}




\begin{tabular}{|c|c|}
\hline Child 4 & $\begin{array}{c}\text { Yes } \square \quad \text { No } \square \\
\text { If yes, date tested: }\end{array}$ \\
\hline Child 5 & $\begin{array}{l}\text { Yes } \square \quad \text { No } \square \\
\text { If yes, date tested: }\end{array}$ \\
\hline Child 6 & $\begin{array}{l}\text { Yes } \square \quad \text { No } \square \\
\text { If yes, date tested: }\end{array}$ \\
\hline
\end{tabular}

7) Employment:

Working full-time $\square \quad$ Working part-time $\square \quad$ Retired $\square \quad$ Disability $\square$ Not Employed $\square$

8) What is/was your job title?

9) What is your average annual income?
a) $0-40 \mathrm{~K}$
b) $40-75 \mathrm{~K}$
c) $\geq 75 \mathrm{~K}$

10) Years of education:
a) High School
b) Some College/University
c) College/University degree
d) Graduate School

11) Ethnicity:
$\square$ White
Black
$\square$ Aboriginal/Native/Indigenous
Asian
Hispanic
Other 
12) In the past six months, how many visits did you make to any of these health professionals?

\begin{tabular}{|c|c|c|c|c|}
\hline & Type of Health Professional & $\begin{array}{r}\text { Was th } \\
\text { schedu } \\
\text { (C) }\end{array}$ & $\begin{array}{l}\text { egularly } \\
\text { leck-up? } \\
\text { one) }\end{array}$ & $\begin{array}{l}\text { Number } \\
\text { of Visits }\end{array}$ \\
\hline$\square$ & $\begin{array}{l}\text { Internist, Family doctor, General } \\
\text { Practitioner }\end{array}$ & Yes $\square$ & No $\square$ & \\
\hline$\square$ & Oncologist & Yes $\square$ & No $\square$ & \\
\hline$\square$ & Surgeon & Yes $\square$ & No $\square$ & \\
\hline$\square$ & Gastrointestinal (GI) Specialist & Yes $\square$ & No $\square$ & \\
\hline$\square$ & Cardiologist & Yes $\square$ & No $\square$ & \\
\hline$\square$ & Emergency Room Visit & Yes $\square$ & No $\square$ & \\
\hline$\square$ & Genetic Counsellor & Yes $\square$ & No $\square$ & \\
\hline$\square$ & $\begin{array}{l}\text { Write in another specialist if not listed } \\
\text { above: }\end{array}$ & Yes $\square$ & No $\square$ & \\
\hline
\end{tabular}


13) Have you ever been diagnosed with ANY type of cancer? $\quad \square$ Yes $\quad \square$ No

If yes, please answer the following:

\begin{tabular}{|l|l|}
\hline \multicolumn{1}{|c|}{ Type of Cancer You Were Diagnosed With } & Age at Diagnosis \\
\hline 1. & \\
\hline 2. & \\
\hline 3. & \\
\hline 4. & \\
\hline 5. & \\
\hline 6. & \\
\hline
\end{tabular}

14) Where do you obtain your general information about Lynch syndrome?

Check all that apply.
1) Family doctor
2) Internet
3) Surgeon
4) Genetic Counsellor
5) Family
6) Newsletter
7) Friends
8) Gastroenterologist

15) Looking at the boxes you just checked, what are the top two sources of information YOU use the most?

1.

2.

16) To manage your health associated with your Lynch syndrome, where do you get information about screening recommendations (e.g., how often to get screened, what kinds of screening)? Check all that apply.
1) Family doctor
2) Internet
3) Surgeon
4) Genetic Counsellor
5) Family
6) Newsletter
7) Friends
8) Gastroenterologist 
17) Looking at the boxes you just checked, what are the top two sources of information YOU use the most?

1.

2.

18) Who (or whose office) follows up with you to manage the booking of your screening procedures associated with your Lynch syndrome? Check all that apply.

\begin{tabular}{|c|}
\hline Family doctor \\
\hline Genetic Counsellor \\
\hline
\end{tabular}

Below is a list of statements that relate to communication with your family doctor about Lynch Syndrome. Please indicate whether or not you agree with each statement (Circle One) or check the Does not apply box.

PICS1) My family doctor asks me whether I agree with his/her decisions Disagree Agree Does not apply regarding management of my Lynch Syndrome.

PICS2) My family doctor gives me a complete explanation for the management of my Lynch Syndrome.

PICS3) My family doctor asks me what I believe is causing my medical symptoms associated with my Lynch Syndrome.

PICS4) My family doctor encourages me to talk about personal concerns related to my Lynch Syndrome.

$\begin{array}{llc}0 & 1 & \square \\ 0 & 1 & \square \\ 0 & 1 & \square \\ 0 & 1 & \square \\ 0 & 1 & \square \\ 0 & 1 & \square \\ 0 & 1 & \square \\ 0 & & \\ & & \square\end{array}$

PICS5) My family doctor encourages me to give my opinion about the management regarding my Lynch Syndrome.

PICS6) I ask my family doctor to explain the procedures associated with aspects of having Lynch Syndrome to me in greater detail.

PICS7) I ask my family doctor for recommendations about managing my Lynch Syndrome.

PICS8) I go into great detail about my Lynch Syndrome during my appointments with my family doctor. 
PICS9) I ask my family doctor a lot of questions about my Lynch Syndrome.

PICS10) I make specific suggestions to my family doctor about ways to manage the risks associated with my Lynch Syndrome.

$0 \quad 1$

PICS11) I insist on a particular kind of test or procedure to manage the risks associated with my Lynch Syndrome.

PICS12) I express doubts about the tests or procedures that my family doctor recommends with regard to my Lynch Syndrome.

PICS13) I give my opinion (agreement or disagreement) about the types of tests or procedures that my family doctor orders for my Lynch Syndrome.

0

0

0

0
1

1

1

1

DMPR1) We would like you to think about your preferences for making decisions about managing Lynch Syndrome. Please read the statements below. Place an $\mathbf{X}$ next to the statement that best describes the way you like your medical decisions made, specifically with regards to your Lynch Syndrome.

A) I like to make the decision about my medical treatment

B) I like to make the decision about my treatment after considering my doctor's opinion

C) I like to share the decision equally with my doctor

D) I like my doctor to make the decision about my medical treatment after considering my opinion.

E) I like my doctor to make the decision about my medical treatment

Please check the appropriate box for each of the following statements.

\begin{tabular}{|l|l|l|l|l|l|}
\hline & $\begin{array}{c}\text { Disagree Very } \\
\text { Much }\end{array}$ & Disagree & $\begin{array}{c}\text { Neither agree or } \\
\text { disagree }\end{array}$ & Agree & $\begin{array}{c}\text { Agree Very } \\
\text { Much }\end{array}$ \\
\hline $\begin{array}{l}\text { TPS1) I doubt that my family } \\
\text { doctor really cares about me as } \\
\text { a person. }\end{array}$ & & & & & \\
\hline $\begin{array}{l}\text { TPS2) My family doctor is } \\
\text { usually considerate of my needs } \\
\text { and puts them first. }\end{array}$ & & & & & \\
\hline $\begin{array}{l}\text { TPS3) I trust my family doctor } \\
\text { so much that I always follow } \\
\text { his/her advice. }\end{array}$ & & & & & \\
\hline $\begin{array}{l}\text { TPS4) If my family doctor tells } \\
\text { me that something is so, then it } \\
\text { must be true. }\end{array}$ & & & & & \\
\hline
\end{tabular}




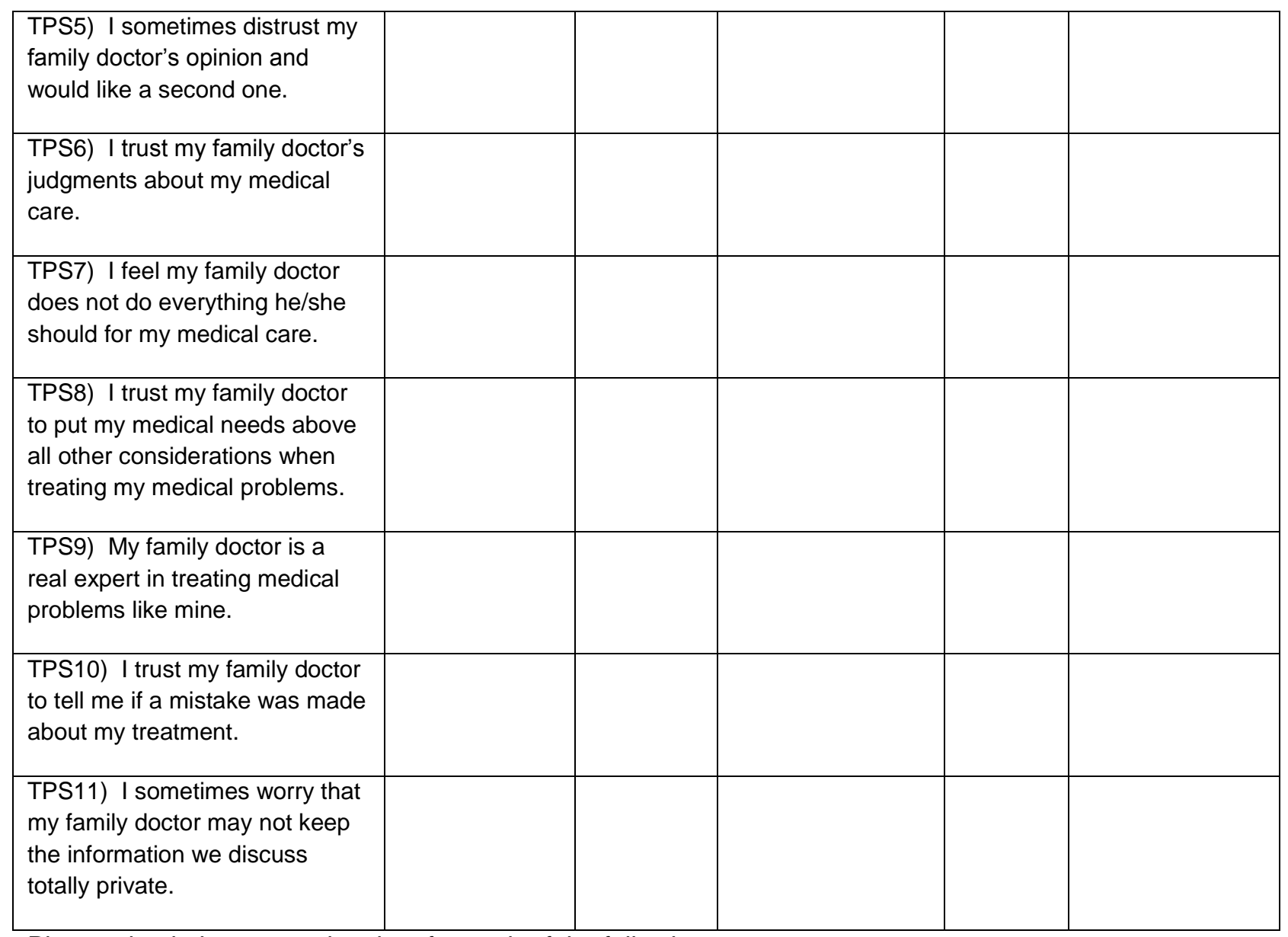

Please check the appropriate box for each of the following statements.

\begin{tabular}{|l|l|l|l|l|}
\hline & $\begin{array}{c}\text { Strongly } \\
\text { Disagree }\end{array}$ & $\begin{array}{c}\text { Slightly } \\
\text { Disagree }\end{array}$ & Slightly Agree & Agree \\
\hline $\begin{array}{l}\text { CASE1) I know that I will be able to } \\
\text { deal with any unexpected health } \\
\text { problems associated with my Lynch } \\
\text { Syndrome }\end{array}$ & & & & \\
\hline $\begin{array}{l}\text { CASE2) I am confident in my ability } \\
\text { to understand written materials } \\
\text { about Lynch Syndrome }\end{array}$ & & & & \\
\hline $\begin{array}{l}\text { CASE3) I am confident in my ability } \\
\text { to understand my doctor's } \\
\text { recommendations about my Lynch } \\
\text { Syndrome }\end{array}$ & & & & \\
\hline $\begin{array}{l}\text { CASE4) It is easy for me to actively } \\
\text { participate in decisions about my } \\
\text { treatment or procedures regarding } \\
\text { my Lynch Syndrome }\end{array}$ & & & & \\
\hline \begin{tabular}{l} 
CASE5) I won't let my Lynch \\
\hline
\end{tabular} & & & & \\
\hline
\end{tabular}




\begin{tabular}{|l|l|l|l|l|}
\hline Syndrome get me down & & & \\
\hline $\begin{array}{l}\text { CASE6) It is easy for me to keep a } \\
\text { positive attitude with regards to } \\
\text { having Lynch Syndrome. }\end{array}$ & & & & \\
\hline $\begin{array}{l}\text { CASE7) It is easy for me to maintain } \\
\text { a sense of humour about having } \\
\text { Lynch Syndrome. }\end{array}$ & & & \\
\hline $\begin{array}{l}\text { CASE8) I am confident that I can } \\
\text { control my negative feelings about } \\
\text { my Lynch Syndrome. }\end{array}$ & & & \\
\hline $\begin{array}{l}\text { CASE9) If I don't understand } \\
\text { something about my Lynch } \\
\text { Syndrome it is easy for me to ask for } \\
\text { help. }\end{array}$ & & & \\
\hline $\begin{array}{l}\text { CASE10) It is easy for me to ask } \\
\text { genetic counsellors questions about } \\
\text { my Lynch Syndrome. }\end{array}$ & & & \\
\hline $\begin{array}{l}\text { CASE11) It is easy for me to ask my } \\
\text { doctor questions about Lynch }\end{array}$ & & & \\
Syndrome. & & & \\
\hline $\begin{array}{l}\text { CASE12) It is easy for me to get } \\
\text { information about Lynch Syndrome. }\end{array}$ & & & & \\
\hline
\end{tabular}

The following statements describe how people may react to the uncertainties of life. Please circle a number ( 1 to 5 ) that best describes to what extent each item is characteristic of you.

IUS1) Unforeseen events upset me greatly.

1

Not at all characteristic of me
3

Somewhat characteristic of me
4
1

5

Not at all characteristic of me
2

Somewhat characteristic of me
3

4

Entirely characteristic of me

IUS3) One should always look ahead so as to avoid surprises.

1

5

Not at all characteristic of me
2

Somewhat characteristic of me

\section{hing \\ IUS4) A small, unforeseen e \\ 5 \\ Not at all characteristic of me \\ 3 \\ Somewhat characteristic of me}

4

Entirely characteristic of me
5

me 
IUS5) I always want to know what the future has in store for me.

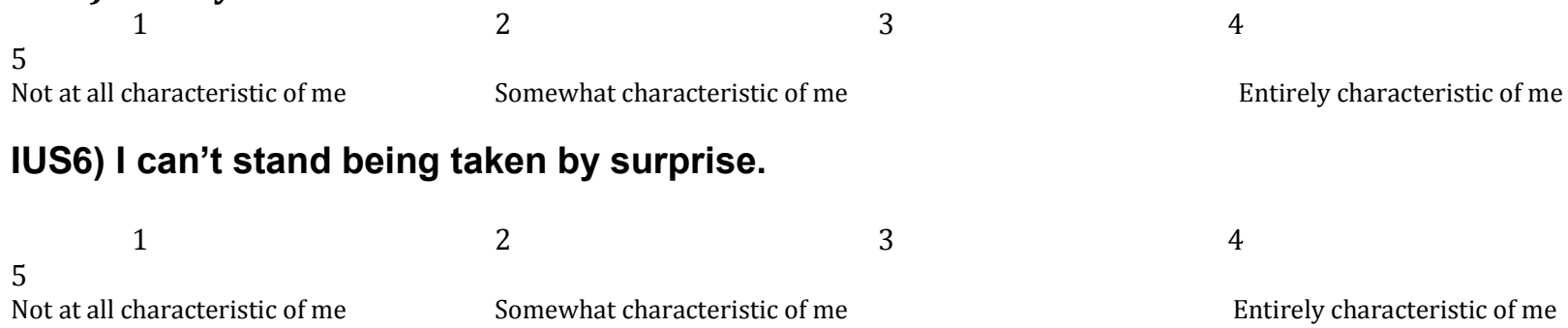

IUS7) I should be able to organize everything in advance.

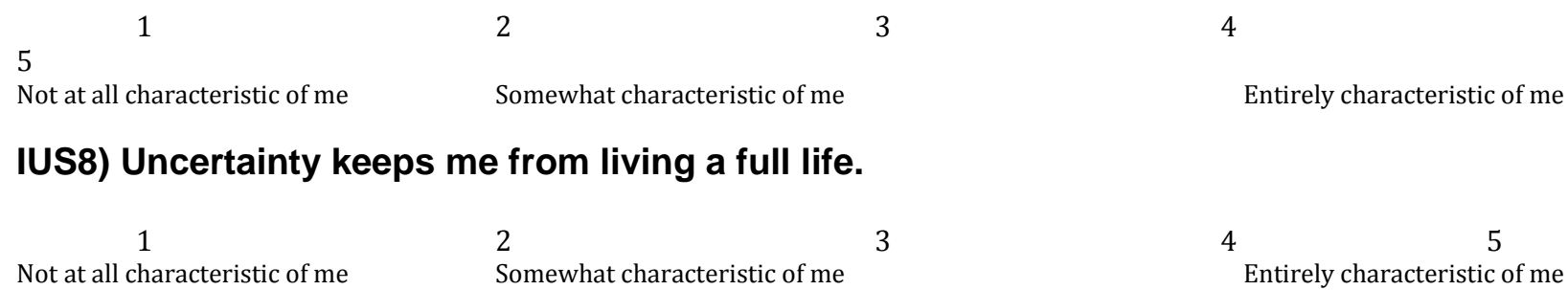

IUS9) When it's time to act, uncertainty paralyzes me.

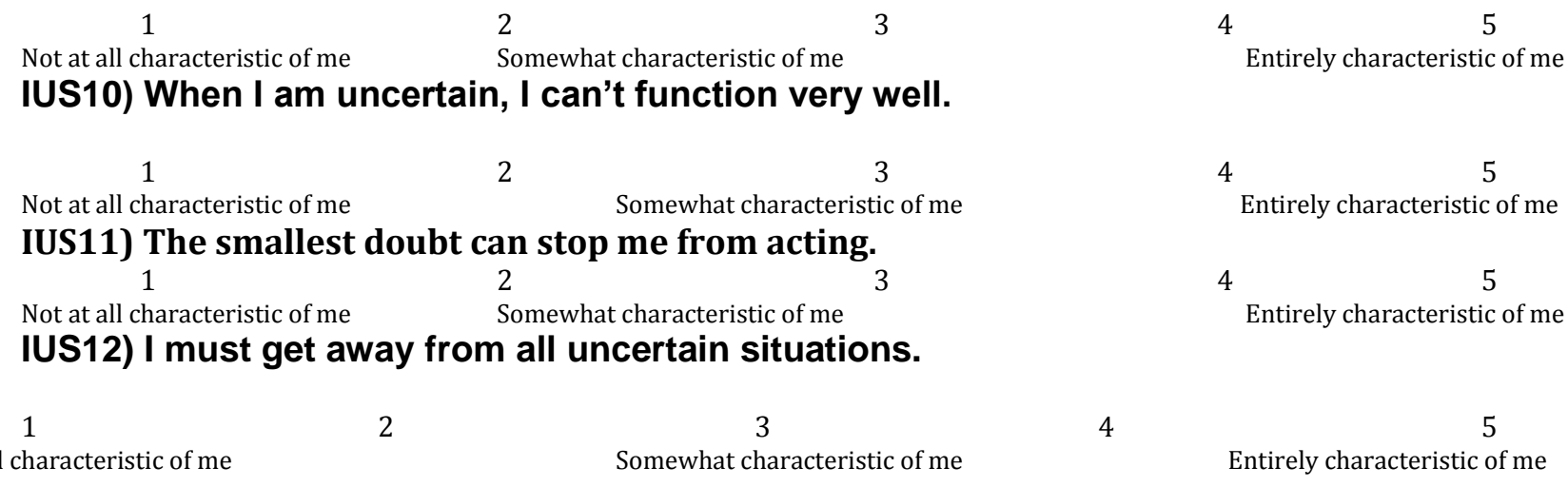


Each question is this section consists of a group of four statements. Please read each group of statements carefully and then select the one which best describes your feelings, over the past six months. Identify the statement by circling the letter next to it, i.e. if you think that statement (a) is correct, circle statement (a); it may be that more than one statement applies, in which case, please ring any that are applicable.

HAl1) (a) I do not worry about my health.

(b) I occasionally worry about my health.

(c) I spend much of my time worrying about my health.

(d) I spend most of my time worrying about my health.

HAl2) (a) I notice aches and pains less than most other people (of my age).

(b) I notice aches and pains as much as most other people (of my age).

(c) I notice aches and pains more than most other people (of my age).

(d) I am aware of aches and pains in my body all the time.

HAI3) (a) As a rule I am not aware of bodily sensations or changes.

(b) Sometimes I am aware of bodily sensations or changes.

(c) I am often aware of bodily sensations or changes.

(d) I am constantly aware of bodily sensations or changes.

HAI4) (a) Resisting thoughts of illness is never a problem.

(b) Most of the time I can resist thoughts of illness.

(c) I try to resist thoughts of illness but am often unable to do so.

(d) Thoughts of illness are so strong that I no longer even try to resist them.

HAI5) (a) As a rule I am not afraid that I have a serious illness.

(b) I am sometimes afraid that I have a serious illness.

(c) I am often afraid that I have a serious illness.

(d) I am always afraid that I have a serious illness.

HAl6) (a) I do not have images (mental pictures) of myself being ill.

(b) I occasionally have images of myself being ill.

(c) I frequently have images of myself being ill.

(d) I constantly have images of myself being ill.

HAI7) (a) I do not have any difficulty taking my mind off thoughts about my health.

(b) I sometimes have difficulty taking my mind off thoughts about my health.

(c) I often have difficulty in taking my mind off thoughts about my health.

(d) Nothing can take my mind off thoughts about my health.

HAI8) (a) I am lastingly relieved if my doctor tells me there is nothing wrong.

(b) I am initially relieved but the worries sometimes return later.

(c) I am initially relieved but the worries always return later.

(d) I am not relieved if my doctor tells me there is nothing wrong. 
HAI9) (a) If I hear about an illness I never think I have it myself.

(b) If I hear about an illness I sometimes think I have it myself.

(c) If I hear about an illness I often think I have it myself.

(d) If I hear about an illness I always think I have it myself.

HAl10) (a) If I have a bodily sensation or change I rarely wonder what it means.

(b) If I have a bodily sensation or change I often wonder what it means.

(c) If I have a bodily sensation or change I always wonder what it means.

(d) If I have a bodily sensation or change I must know what it means.

HAl11) (a) I usually feel at very low risk for developing a serious illness.

(b) I usually feel at fairly low risk for developing a serious illness.

(c) I usually feel at moderate risk for developing a serious illness.

(d) I usually feel at high risk for developing a serious illness.

HAl12) (a) I never think I have a serious illness.

(b) I sometimes think I have a serious illness.

(c) I often think I have a serious illness.

(d) I usually think that I am seriously ill.

HAl13) (a) If I notice an unexplained bodily sensation I don't find it difficult to think about other things.

(b) If I notice an unexplained bodily sensation I sometimes find it difficult to think about other things

(c) If I notice an unexplained bodily sensation I often find it difficult to think about other things.

(d) If I notice an unexplained bodily sensation I always find it difficult to think about other things.

HAl14) (a) My family friends would say I do not worry enough about my health.

(b) My family friends would say I have a normal attitude to my health.

(c) My family friends would say I worry too much about my health.

(d) My family friends would say I am a hypochondriac.

HAl15) (a) If I had a serious illness I would still be able to enjoy things in my life quite a lot.

(b) If I had a serious illness I would still be able to enjoy things in my life a little.

(c) If I had a serious illness I would be almost completely unable to enjoy things in my life.

(d) If I had a serious illness I would be completely unable to enjoy life at all.

HAl16) (a) If I developed a serious illness there is a good chance that modern medicine would be able to cure me.

(b) If I developed a serious illness there is a moderate chance that modern medicine would be able to cure me.

(c) If I developed a serious illness there is a very small chance that modern medicine would be able to cure me.

(d) If I developed a serious illness there is no chance that modern medicine would be able to cure me. 
HAl17) (a) A serious illness would ruin some aspects of my life.

(b) A serious illness would ruin many aspects of my life.

(c) A serious illness would ruin almost every aspect of my life.

(d) A serious illness would ruin every aspect of my life.

HAl18) (a) If I had a serious illness I would not feel that I had lost my dignity.

(b) If I had a serious illness I would feel that I had lost a little of my dignity.

(c) If I had a serious illness I would feel that I had lost quite a lot of my dignity.

(d) If I had a serious illness I would feel that I had totally lost my dignity. 
These questions will help your physician to know how you are feeling. Read every sentence. Place an " $X$ " on the answer that best describes how you have been feeling during the LAST WEEK. You do not have to think too much to answer. In this questionnaire, spontaneous answers are important.

HADS1) I feel tense or wound up

$\square$ Most of the time

$\square$ A lot of the time

$\square$ From time to time

$\square$ Not at all

HADS3) I still enjoy the things I used to enjoy

$\square$ Definitely as much

$\square$ Not quite so much

$\square$ Only a little

$\square$ Hardly at all

HADS5) I get a sort of frightened feeling as if something awful is about to happen

$\square$ Very definitely and quite badly

$\square$ Yes, but not too badly

$\square$ A little, but it doesn't worry me

$\square$ Not at all

HADS7) I can laugh and see the funny side of things

$\square$ As much as I always could

$\square$ Not quite as much now

$\square$ Definitely not so much now

$\square$ Not at all

HADS9) Worrying thought goes through my mind $\square$ A great deal of the time

$\square$ A lot of the time

$\square$ From time to time but not too often

$\checkmark$ Only occasionally

HADS11) I feel cheerful

$\square$ Not at all

$\square$ Not often

$\square$ Sometimes

$\square$ Most of the time

HADS13) I can sit at ease and feel relaxed

$\square$ Definitely

$\square$ Usually

$\square$ Not often

$\square$ Not at all
HADS2) I feel as though I am slowed down

$\square$ Nearly all the time

$\square$ Very often

$\square$ Sometimes

$\square$ Not at all

HADS4) I get a sort of frightened feeling like butterflies in the stomach

$\square$ Not at all

$\square$ Occasionally

$\square$ Quite often

$\square$ Very often

HADS6) I have lost interest in my appearance

$\square$ Definitely

$\square$ I don't take so much care as I should

$\checkmark$ I may not take quite as much care

$\square$ I take just as much care as ever

HADS8) I feel restless, as if I have to be on the move

$\square$ Very much indeed

$\square$ Quite a lot

$\square$ Not very much

$\square$ Not at all

HADS10) I look forward with enjoyment to things

$\square$ As much as I ever did

$\square$ Rather less than I used to

$\square$ Definitely less than I used to

$\square$ Hardly at all

HADS12) I get sudden feelings of panic

$\square$ Very often indeed

$\square$ Quite often

$\square$ Not very often

$\square$ Not at all

HADS14) I can enjoy a good TV or radio program

or

book

$\square$ Often

$\square$ Sometimes

$\square$ Not often

$\square$ Very seldom 
ODHP1) I talk as little as possible about my Lynch syndrome because I don't want to make my family uneasy.

$\begin{array}{llll}\text { Strongly Agree } & \text { Agree } & \text { Disagree } & \text { Strongly Disagree }\end{array}$

ODHP2) My partner doesn't like me to talk about my Lynch syndrome.

$\begin{array}{llll}\text { Strongly Agree } & \text { Agree } & \text { Disagree } & \text { Strongly Disagree }\end{array}$

ODHP3) My children don't like me to talk about my Lynch syndrome.
Strongly Agree
Agree
Disagree
Strongly Disagree
N/A (no children)

ODHP4) If I talk about my Lynch syndrome, others gloss over it.
Strongly Agree
Agree
Disagree
Strongly Disagree

ODHP5) My family always wants to hear from me that I am doing well.
Strongly Agree
Agree
Disagree
Strongly Disagree

ODHP6) Talking about emotions related to my Lynch syndrome upsets my family.

$\begin{array}{llll}\text { Strongly Agree } & \text { Agree } & \text { Disagree } & \text { Strongly Disagree }\end{array}$

ODHP7) My partner often doesn't know what to say or to do when I'm feeling down (because of my Lynch syndrome).

$\begin{array}{llll}\text { Strongly Agree } & \text { Agree } & \text { Disagree } & \text { Strongly Disagree }\end{array}$

ODHP8) My children often don't know what to say or to do when I'm feeling down (because of my Lynch syndrome).

Strongly Agree Agree Disagree Strongly Disagree N/A (no children)

ODHP9) I am mostly the one who starts a conversation in the family about my Lynch Syndrome and problems.

Strongly Agree

Agree

Disagree

Strongly Disagree 


\section{CHI1) Please list your most recent illness or injury:}

The following are ways of reacting to health problems, such as illnesses, sickness, or injuries. We are interested in your last illness, sickness, or injury. Please circle a number from 1 to 5 for each of the following items. Indicate how much you engaged in these types of you encountered this health problem.

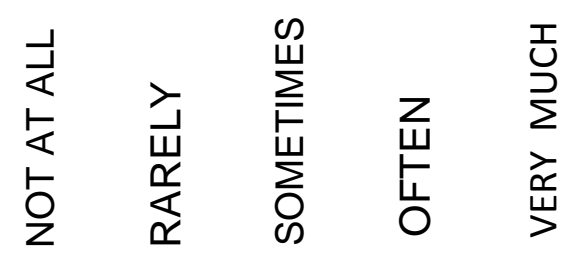

CHI2) Think about better times........................ 1 2 $34 \quad 4 \quad 5$

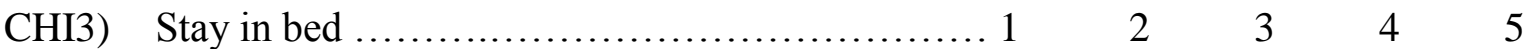

CHI4) Find out more information..................... 1 2 $24 \begin{array}{llll}3 & 4 & 5\end{array}$

CHI5) Wonder "why me" .............................. 1 2 $34 \begin{array}{llll} & 3\end{array}$

CHI6) $\quad$ Be with others .................................

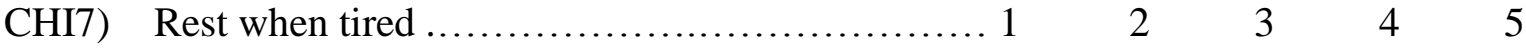

CHI8) Seek treatment quickly.......................... 1 2 $20 \begin{array}{llll}3 & 4 & 5\end{array}$

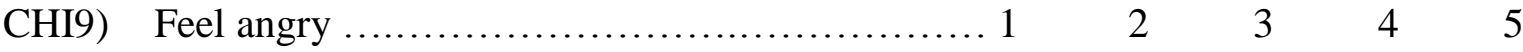

CHI10) Daydream .................................. 1 2 $24 \begin{array}{llll} & 3 & 4 & 5\end{array}$

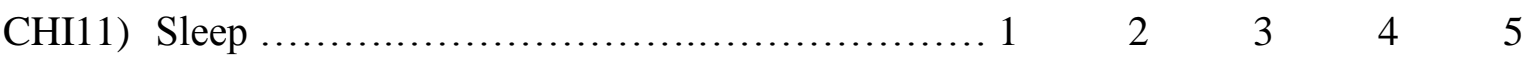

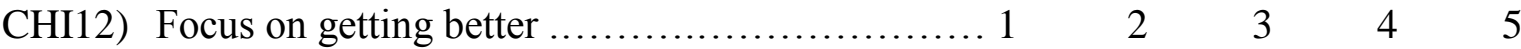

CHI13) Become frustrated ............................

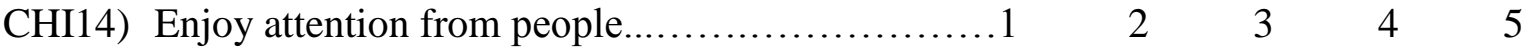

CHI15) Conserve energy................................ 1 2 $24 \begin{array}{lll}3 & 4 & 5\end{array}$

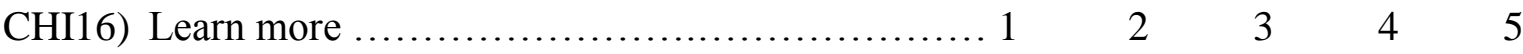

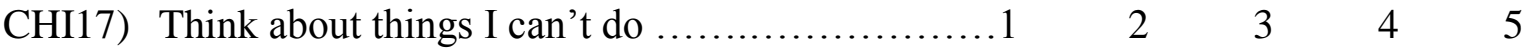

CHI18) Plan for the future ............................... 
CHI19) Stay warm

CHI20) Comply with advice.......................... $1 \quad 2 \quad 3 \quad 4 \quad 5$

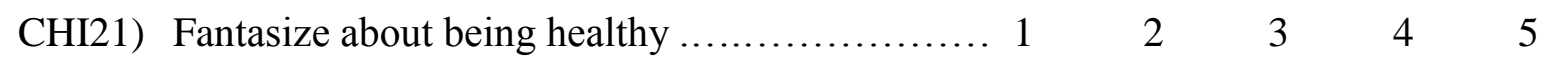

CHI22) Listen to music................................ 1 2 $20 \begin{array}{llll} & 3 & 4 & 5\end{array}$

CHI23) Make surroundings quiet........................ 1 2 $20 \begin{array}{llll} & 3 & 4 & 5\end{array}$

CHI24) Follow doctor's advice........................... $1 \quad 2 \quad 2 \quad 3 \quad 4 \quad 5$

CHI25) Wish it hadn't happened........................ 1 2 $20 \begin{array}{llll}3 & 4 & 5\end{array}$

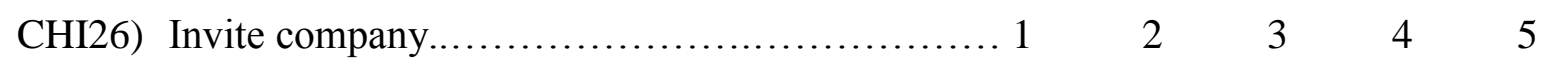

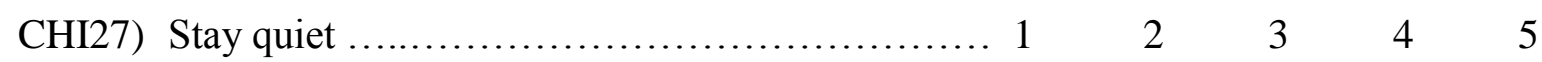

CHI28) Take medications on time......................... $1 \quad 2 \quad 3 \quad 4 \quad 5$

CHI29) Think about being vulnerable..................... 1 2 $24 \begin{array}{llll}3 & 4 & 5\end{array}$

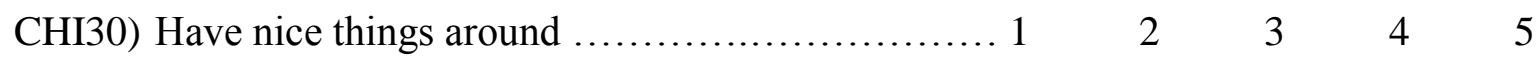

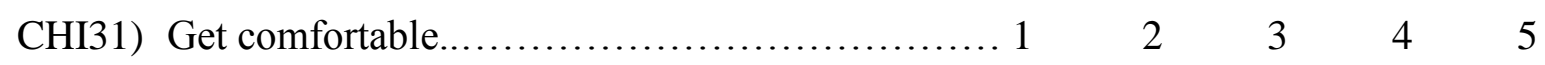

CHI32) Find out about treatments........................ 1 2 $24 \begin{array}{llll} & 3 & 4 & 5\end{array}$

CHI33) Worry about my health.......................... 1 2 $3 \begin{array}{llll} & 3 & 4 & 5\end{array}$ 
Please circle your answers in the boxes provided below:

\begin{tabular}{|c|c|c|c|c|c|}
\hline & Not at all & A little & Somewhat & Quite a bit & $\overline{\text { A lot }}$ \\
\hline $\begin{array}{l}\text { WIS1) Fears of developing cancer have affected } \\
\text { my relationships with others }\end{array}$ & 0 & 1 & 2 & 3 & 4 \\
\hline $\begin{array}{l}\text { WIS2) Thoughts of Lynch syndrome have affected } \\
\text { my ability to sleep }\end{array}$ & 0 & 1 & 2 & 3 & 4 \\
\hline $\begin{array}{l}\text { WIS3) I am able to talk to my partner about my } \\
\text { Lynch syndrome concerns }\end{array}$ & 0 & 1 & 2 & 3 & 4 \\
\hline $\begin{array}{l}\text { WIS4) My partner is able to understand my } \\
\text { concerns }\end{array}$ & 0 & 1 & 2 & 3 & 4 \\
\hline $\begin{array}{l}\text { WIS5) Thoughts of Lynch syndrome have affected } \\
\text { my work }\end{array}$ & 0 & 1 & 2 & 3 & 4 \\
\hline $\begin{array}{l}\text { WIS6) I think about my } \\
\text { children's/grandchildren's chances of developing } \\
\text { cancer or Lynch syndrome }\end{array}$ & 0 & 1 & 2 & 3 & 4 \\
\hline $\begin{array}{l}\text { WIS7) Concerns about Lynch syndrome or cancer } \\
\text { have affected my ability to have fun }\end{array}$ & 0 & 1 & 2 & 3 & 4 \\
\hline $\begin{array}{l}\text { WIS8) Fears of Lynch syndrome have affected my } \\
\text { ability to feel sexually attractive }\end{array}$ & 0 & 1 & 2 & 3 & 4 \\
\hline $\begin{array}{l}\text { WIS9) Worries about Lynch syndrome have } \\
\text { affected my ability to meet the needs of my family }\end{array}$ & 0 & 1 & 2 & 3 & 4 \\
\hline $\begin{array}{l}\text { WIS10) Lynch syndrome concerns have affected } \\
\text { my ability to concentrate }\end{array}$ & 0 & 1 & 2 & 3 & 4 \\
\hline $\begin{array}{l}\text { WIS11) Cancer risks affected my decision to have } \\
\text { children }\end{array}$ & 0 & 1 & 2 & 3 & 4 \\
\hline
\end{tabular}

\section{Thank you for participating!}

If you would like to make any additional comments, please feel free to do so in the space provided. 


\section{Appendix B}

\section{Study II Semi-Structured Interview Guide}

Participant ID \#

Date of Interview

"We are very happy that you've agreed to talk to us, thank you again! We would like to talk to you about your experience of being a parent who has been diagnosed with Lynch Syndrome."

\section{Interview Guide}

\section{Demographic Questions}

a. How old are you?

b. Are you currently in a relationship? If yes, how long have you been in your relationship?

c. Who do you currently live with?

d. How many children do you have? What are their ages? Do they still live at home?

e. Can you tell me a bit about your experience with Lynch Syndrome?

Probing Questions: When did you receive your diagnosis? How did you react when you received the diagnosis? How are you coping with the diagnosis now?

\section{Section 1: Perceptions of Self}

a. How would you describe your own health?

b. How often do you think about (or worry about) your health?

c. When does this worry tend to come up?

d. When you worry about your health, what types of things do you do that make your worry better?

e. What types of thinks make your worry worse? 
f. Sometimes when people are diagnosed with Lynch Syndrome, it changes the way they think about themselves. What has your experience been with this? Can you describe the change?

g. Sometimes when people are diagnosed with Lynch Syndrome, it changes the way they think about their role as parents? What has your experience been with this? Can you describe the change?

h. When you compare yourself to other people your own age, how does your health compare? To what extent does having Lynch Syndrome set you apart from your peers?

i. What about Lynch Syndrome are you most worried about with regards to the future?

Probing Questions: What else worries you about the future? What is the worst part of that for you?

\section{Section 2: Passing Down Lynch Syndrome to Children}

a. As you are probably aware, Lynch Syndrome can be passed down to one's children.

Do you ever think about this?

Probing Questions: When do you think about this? How often?

b. How do you feel when you think about passing on Lynch Syndrome to your child(ren)?

Emotional Prompts: Indifferent, guilty, depressed, anxious, terrified, happy, relieved

c. Have any of your children been tested for Lynch Syndrome?

If yes, what were the results?

i. How did you feel when you found out the results?

If no, what is the reason they haven't been tested?

i. Do you think you will have them tested in the future?

ii. How do you think you would feel if you found out they do not have Lynch Syndrome?

iii. How do you think you would feel if you found out they do have Lynch Syndrome?

d. Do you have any regrets about your children being tested/not tested? 


\section{Section 3: Perceptions of Children}

a. How would you describe your children's health?

b. Do you worry about your children's health?

c. What specific concerns do you have?

d. When you are worried about your children's health, what types of things make this worry better? What types of things make this worry worse?

e. Does knowing that you child could be (or is) a carrier of Lynch Syndrome change the way you think about their health?

\section{Wrapping Up}

a. As a parent who has Lynch Syndrome, what do you know now that you wish you knew earlier?

b. Is there anything you would have done differently?

c. What advice would you give to other parents who are in your shoes?

d. Those are all of the formal questions I have for you. Is there anything else about your experience as a parent with Lynch Syndrome that you think would be important for me to know?

e. Do you have any questions for me?

"Thank you so much for participating in this interview - your responses have been very helpful. As a thank you gift, I will be mailing you a $\mathbf{\$ 4 0}$ gift card shortly. If you have any questions or want to get in touch with the study for any reason, please give us a call - the number is 1-866-643-7604." 


\section{Appendix C}

\section{Study II Codebook}

\section{Template Coding Sheet for Phase II Interviews}

\section{Perceptions of Self}

\begin{tabular}{l} 
Appraisal of Personal Health \\
-parent describes their own health \\
-talks about personal vulnerability to illness (e.g., risk perception, likelihood of getting \\
cancer) \\
-compares own health to health of peers \\
Positive \\
-parent describes themselves as healthy, or states that health is good or \\
fine \\
-parent states that their health is comparable to peers or better than peers \\
Negative \\
-parent describes themselves as unhealthy, or describes medical \\
symptoms, complaints, etc. \\
-parent states that their health is worse than their peers \\
Wersonal Health \\
-parent describes how often/how much time they spend worrying about their own health, \\
their future, etc. \\
Low \\
-Parent states that they do not worry about their health \\
- Parent states that they used to worry about their health but that they no \\
longer worry \\
Moderate \\
-Parent states that they worry "somewhat", "a bit" or "sometimes" about \\
their health \\
-Parent states that they worry when they notice a symptom, are cued to \\
think about their health (E.g., news report about cancer), or have an \\
upcoming appointment \\
High \\
\hline
\end{tabular}




\begin{tabular}{|c|}
\hline $\begin{array}{l}\text { Feared Consequences } \\
\text {-parent describes the feared consequences of developing cancer/of current cancer } \\
\text {-include knowledge gained from past experience with cancer (e.g., family history, early } \\
\text { health related experiences, illness in peer groups, info in media) } \\
\text {-e.g., going through treatment, death, financial concerns, impact on family members, etc. }\end{array}$ \\
\hline $\begin{array}{l}\text { Cancer } \\
\text {-includes fear of developing cancer, symptoms associated with cancer, } \\
\text { symptoms associated with consequences of undergoing treatment for } \\
\text { cancer, etc. }\end{array}$ \\
\hline $\begin{array}{l}\text { Death } \\
\text {-includes fear of dying } \\
\text {-DO NOT CODE If parent talks about fear of dying in reference to the } \\
\text { impact it has on family (“I am afraid that I will die and abandon my kids) } \\
\text { - this goes in Family Impact code }\end{array}$ \\
\hline $\begin{array}{l}\text { Family Impact } \\
\text {-parent refers to the impact cancer or death would have on family } \\
\text { members } \\
\text {-e.g., "If I can't work, I won't be able to provide for my family", "I don't } \\
\text { want my kids to see me sick", "I don't want to die and leave my child } \\
\text { without a parent." }\end{array}$ \\
\hline $\begin{array}{l}\text { Coping Strategies } \\
\text {-Parent reports engaging in behaviours/strategies to manage anxiety about their own } \\
\text { health }\end{array}$ \\
\hline $\begin{array}{l}\text { Solution Focused } \\
\text {-Includes active coping strategies, making a plan of action and sticking to } \\
\text { it ("I've been concentrating my efforts on doing something about the } \\
\text { situation I'm in") } \\
\text {-e.g., becoming informed about Lynch syndrome, making healthy lifestyle } \\
\text { changes, getting instrumental support, seeing physician, etc. }\end{array}$ \\
\hline $\begin{array}{l}\text { Emotional Support and Acceptance Based } \\
\text {-Includes using emotional support ("I talk to my family about my } \\
\text { worries"), acceptance of the situation ("I have no control over what will } \\
\text { happen, so I have to accept it), positive reappraisal of the situation (e.g., to } \\
\text { change or grow as a result of the situation), and active cognition (e.g., } \\
\text { prayer) }\end{array}$ \\
\hline $\begin{array}{l}\text { Dysfunctional } \\
\text {-includes denial (“I tell myself it's nothing"), avoidance (of cues that } \\
\text { trigger anxiety - e.g., doctors, family members, health information), self- } \\
\text { criticism or self-blame }\end{array}$ \\
\hline
\end{tabular}




\section{Perceptions of Passing down Lynch Syndrome to Child}

\section{Worry about Child having Lynch syndrome}

-Parent describes how often/how much time they spend thinking about Lynch being passed down to children/grandchildren

\section{Low}

-Parent states that they do not think about passing down the gene to children/grandchildren

- Parent states that they used to think about passing down the gene but they no longer do

\section{Moderate}

-Parent states that they think "somewhat", "a bit" or "sometimes" about passing down the gene to children/grandchildren

-Parent states that they think about it only when they are cued to the topic (e.g., genetic testing appointment)

\section{High}

-Parent states that they think "a lot", "always", "constantly", or "too much" about their passing down the gene

\section{Emotional Reaction}

- code any feelings reported when thinking of passing down gene to child/genetic testing of children (e.g., sadness, guilt, anxiety)

- include the reactions of parents who have been through the experience and the predicted reactions of parents whose children haven't been tested yet -participant does not need to explicitly label the emotion. If they provide a thought that is clearly linked to an emotion, this can be coded (E.g., "I just wish that it wasn't my fault that this was passed down" would be coded as Guilt/Responsibility)

\section{Guilt}

-parent reports feeling guilty about passing down gene

-parent describes feeling responsible for this condition being in the family

\section{Sadness}

-parent reports feeling upset/sad about passing on LS

-parent talks about the unpleasant/negative life events children will have to endure because of this (e.g., go through screenings, could get cancer, etc.)

\section{Anger}

-parent talks about being mad/angry about passing on LS

-parent talks about the unfairness/unjustness of the situation (e.g., "It isn't fair - they didn't do anything to deserve this) 


\begin{tabular}{|c|}
\hline $\begin{array}{l}\text { Anxiety } \\
\text {-parent talks about being scared/worried/anxious when thinking about } \\
\text { passing down gene } \\
\text {-parent talks about what scares them about the situation }\end{array}$ \\
\hline $\begin{array}{l}\text { Happiness } \\
\text {-parent talks about experiencing happiness or having a sense of relief after } \\
\text { finding out test results }\end{array}$ \\
\hline $\begin{array}{l}\text { Effects of Genetic Testing } \\
\text {-parent talks about the benefits/consequences of having children/grandchildren tested } \\
\text { (e.g., cancer screening procedures, better to know than not know, etc.) }\end{array}$ \\
\hline $\begin{array}{l}\text { Increased Sense of Control } \\
\text {-parent talks about having more control over the situation } \\
\text { - having a game plan to follow } \\
\text {-better to know than to not know } \\
\text {-ability to be proactive } \\
\text {-ability to be informed }\end{array}$ \\
\hline $\begin{array}{l}\text { Enhanced Medical Access } \\
\text {-parent talks about factors related to health care } \\
\text {-e.g., better screening, more access to physicians }\end{array}$ \\
\hline $\begin{array}{l}\text { Emotional/Psychological Consequences } \\
\text {-parent talks about emotional or psychological benefits/consequences of } \\
\text { testing } \\
\text {-e.g., gives them peace of mind, places emotional burden on children }\end{array}$ \\
\hline $\begin{array}{l}\text { Practical Concerns } \\
\text {-parent talks about practical concerns they have related to the life of their } \\
\text { children and how genetic testing could affect their lives. } \\
\text {-e.g., access to insurance }\end{array}$ \\
\hline 3. Perceptions of Child \\
\hline $\begin{array}{l}\text { Appraisal of Child's Health } \\
\text {-parent describes their child's health } \\
\text {-talks about their children's vulnerability to illness (e.g., likelihood of having Lynch } \\
\text { syndrome or getting cancer) } \\
\text {-compares child's health to health of peers }\end{array}$ \\
\hline $\begin{array}{l}\text { Positive Health } \\
\text {-parent describes their child as healthy, or states that health is good or fine } \\
\text {-parent states that their child's health is comparable to/better than peers }\end{array}$ \\
\hline
\end{tabular}




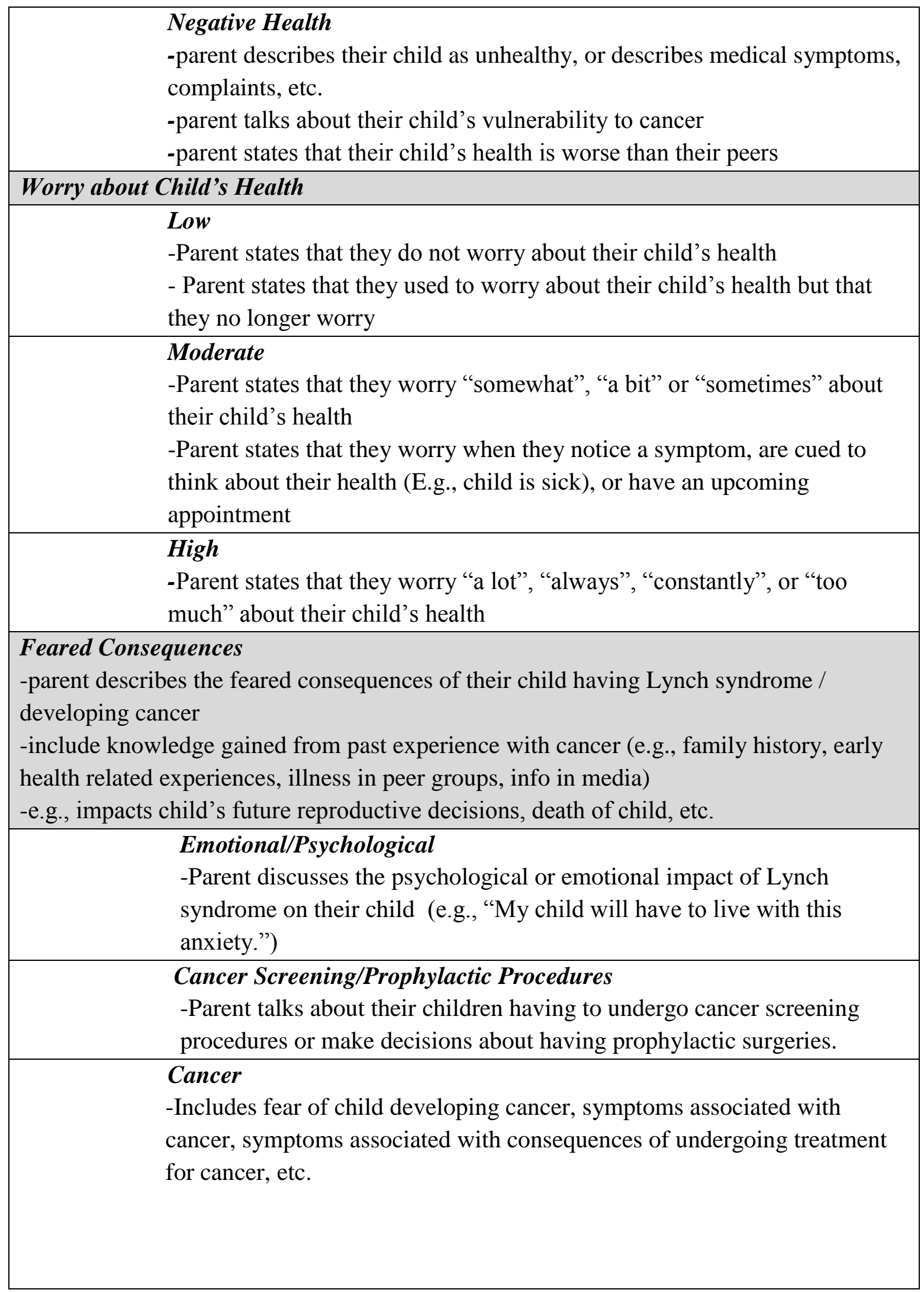




\section{Coping Strategies}

-Parent reports engaging in behaviours/strategies to manage anxiety about their child's health

\section{Solution Focused}

-Includes active coping strategies, making a plan of action and sticking to it ("I've been concentrating my efforts on doing something about the situation I'm in")

-e.g., becoming informed about Lynch syndrome, making healthy lifestyle changes, getting instrumental support, taking child to see a physician, etc.

\section{Emotional Support and Acceptance Based}

-Includes using emotional support ("I talk to my child about my worries"), acceptance of the situation ("I have no control over what will happen, so I have to accept it), positive reappraisal of the situation (e.g., to change or grow as a result of the situation), and active cognition (e.g., prayer)

\section{Dysfunctional}

-includes denial ("I tell myself it's nothing"), avoidance (of cues that trigger anxiety - e.g., doctors, family members, health information), selfcriticism or self-blame 


\section{References}

Aarnio, M., Sankila, R., Pukkala, E., Salovaara, R., Aaltonen, L. A., de la Chapelle, A., ... Järvinen, H. J. (1999). Cancer risk in mutation carriers of DNA-mismatchrepair genes. International Journal of Cancer, 81, 214-218. doi:10.1002/(SICI)1097-0215(19990412)81:2<214::AID-IJC8>3.0.CO;2-L

Abramowitz, J. S., Deacon, B. J., \& Valentiner, D. P. (2007). The short health anxiety inventory: Psychometric properties and construct validity in a non-clinical sample. Cognitive Therapy and Research, 31(6), 871-883. doi: http://dx.doi.org/10.1007/s10608-006-9058-1

Abramowitz, J. S., Olatunji, B. O., \& Deacon, B. J. (2007). Health anxiety, hypochondriasis, and the anxiety disorders. Behavior Therapy, 38, 86-94. doi: 10.1016/j.beth.2006.05.001

Afilalo, J., Marinovich, A., Afilalo, M., Colacone, A., Leger, R., Unger, B., et al. (2004). Nonurgent emergency department patient characteristics and barriers to primary care. Academic Emergency Medicine, 11, 1302-1310. doi:

10.1197/j.aem.2004.08.032

Aktan-Collan, K., Haukkala, A., Mecklin, J., Uutela, A., \& Kääriäinen, H. (2001). Psychological consequences of predictive genetic testing for hereditary nonpolyposis colorectal cancer (HNPCC): A prospective follow-up study. International Journal of Cancer, 93, 608-611. doi:10.1002/ijc.1372

Aktan-Collan, K., Kääriäinen, H., Järvinen, H., Peltomäki, P., Pylvänäinen, K., Mecklin, J. P., \& Haukkala, A. (2013). Psychosocial consequences of predictive genetic 
testing for Lynch syndrome and associations to surveillance behaviour in a 7-year follow-up study. Familial Cancer, 12, 639-646. doi: 10.1007/s10689.013-9628-9

Alberts, N. M., Hadjistavropoulos, H. D., Jones, S. L., \& Sharpe, D. (2013). The short health anxiety inventory: A systematic review and meta-analysis. Journal of Anxiety Disorders, 27(1), 68-78. doi:

http://dx.doi.org/10.1016/j.janxdis.2012.10.009

American Cancer Society. (2011). Colorectal Cancer Facts \& Figures 2011-2013. Atlanta, GA: American Cancer Society.

American Gastroenterological Association. (2013, September). Patient centre. Retrieved from http://www.gastro.org/patient-center

American Psychiatric Association. (2013). Diagnostic and Statistical Manual of Mental Disorders ( $5^{\text {th }}$ ed.). Arlington, VA: American Psychiatric Publishing.

Apse, K. A., Biesecker, B. B., Giardiello, F. M., Fuller, B. P., \& Bernhardt, B. A. (2004). Perceptions of genetic discrimination among at-risk relatives of colorectal cancer patients. Genetics in Medicine, 6, 510-516. doi:

10.1097/01.GIM.0000144013.96456.6C

Arver, B., Haegermark, A., Platten, U., Lindblom, A., \& Brandberg, Y. (2004).

Evaluation of Psychosocial Effects of Pre-Symptomatic Testing for Breast/Ovarian and Colon Cancer Pre-Disposing Genes: A 12-Month Follow-Up. Familial Cancer, 3, 109-116. doi:10.1023/B:FAME.0000039863.89137.f9

Asmundson, G., Abramowitz, J., Richter, A., \& Whedon, M. (2010). Health Anxiety: Current Perspectives and Future Directions. Current Psychiatry Reports, 12, 306312. doi:10.1007/s11920-010-0123-9 
Asmundson, G., Taylor, S., \& Cox, B. J. (2001). Health anxiety: Clinical and research perspectives on hypochondriasis and related conditions. Toronto, ON: John Wiley \& Sons.

Backman, A. Blomqvist, P., Lagerlund, M., Carlsson-Holm, E., \& Adami, J. (2008). Characteristics of non-urgent patients: Cross-sectional study of emergency department and primary care patients. Scandinavian Journal of Primary Health Care, 26, 181-187. doi:10.1080/02813430802095838

Barrera, M., D'Agostino, N. M., Gibson, J., Gilbert, T., Weksberg, R., \& Malkin, D. (2004). Predictors and mediators of psychological adjustment in mothers of children newly diagnosed with cancer. Psycho-Oncology, 13, 630-641. doi:http://dx.doi.org/10.1002/pon.765

Barsky, A.J., Coeytaux, R. R., Sarnie, M. K., \& Cleary, P.D. (1993). Hypochondriacal patients' beliefs about good health. American Journal of Psychiatry, 150, 10851089.

Barsky, A. J., Delamater, B. A., \& Orav, J. E. (1999). Panic disorder patients and their medical care. Psychosomatics, 40, 50-56. doi: 10.1016/S0033-3182(99)71271-5

Barsky, A. J., Ettner, S. L., Horsky, J., \& Bates, D. W. (2001). Resource Utilization of Patients with Hypochondriacal Health Anxiety and Somatization. Medical Care, 39, 705-715. doi: 10.1097/00005650-200107000-00007

Barsky, A. J., Fama, J. M., Bailey, E. D., \& Ahern, D. K. (1998). A Prospective 4- to 5Year Study of DSM-III-R Hypochondriasis. Archives of General Psychiatry, 55, 737-744. doi:10.1001/archpsyc.55.8.737 
Bartuma, K., Nilbert, M., \& Carlsson, C. (2012). Family perspectives in Lynch syndrome becoming a family at risk, patterns of communication and influence on relations. Hereditary Cancer in Clinical Practice, 10, 6. doi:10.1186/1897-4287-10-6

Beland, F., Lemay, A. Boucher, M. (1998). Patterns of visits to hospital-based emergency rooms. Social Science and Medicine, 47, 165-179. doi: 10.1016/S02779536(98)00029-X

Bhandari, A., \& Wagner, T. (2006). Self-reported utilization of health care services: Improving measurement and accuracy. Medical Care Research and Review, 63, 217-235. doi: $10.1177 / 1077558705285298$

Bjelland, I., Dahl, A. A., Haug, T. T., \& Neckelmann, D. (2002). The validity of the Hospital Anxiety and Depression Scale: An updated literature review. Journal of Psychosomatic Research, 52, 69-77. doi: 10.1016/S0022-3999(01)00296-3

Bleichhardt, G., \& Hiller, W. (2007). Hypochondriasis and health anxiety in the German population. British Journal of Health Psychology, 12, 511-523. doi:10.1348/135910706X146034

Bleiker, E. M. A., Hahn, D. E. E., \& Aaronson, N. K. (2003). Psychosocial issues in cancer genetics. Acta Oncologica, 42, 276-286. doi:10.1080/02841860310004391

Bonadona, V., Saltel, P., Desseigne, F., Mignotte, H., Saurin, J.-C., Wang, Q., ... Lasset, C. (2002). Cancer patients who experienced diagnostic genetic testing for cancer susceptibility: Reactions and behavior after the disclosure of a positive test result. Cancer Epidemiology Biomarkers \& Prevention, 11, 97-104. 
Broadstock, M., Michie, S., \& Marteau, T. (2000). Psychological consequences of predictive genetic testing: A systematic review. European Journal of Human Genetics, 8, 731-738. doi:10.1038/sj.ejhg.5200532

Carlsson, C., \& Nilbert, M. (2007). Living with hereditary non-polyposis colorectal cancer: Experiences from and impact of genetic testing. Journal of Genetic Counseling, 16, 811-820. 10.1007/s10897-007-9117-0

Carroll, B. T., Kathol, R. G., Noyes Jr., R., Wald, T. G., \& Clamon, G. H. (1993). Screening for depression and anxiety in cancer patients using the Hospital Anxiety and Depression Scale. General Hospital Psychiatry, 15, 69-74. doi:10.1016/0163-8343(93)90099-A

Carver, C.S., (1997). You want to measure coping but your protocol's too long: Consider the brief COPE. International Journal of Behavioral Medicine, 4, 92100. 10.1207/s15327558ijbm0401_6

Chalder, T., McCrone, P., Darnley, S., Knapp, M., Jones, R. H., \& Wessely, S. (2006). Cognitive behavioural therapy in addition to antispasmodic therapy for irritable bowel syndrome in primary care: Randomized controlled trial. Health Technology Assessment, 10, 1-67. doi: 10.3310/hta10190

Claes, E., Denayer, L., Evers, Kiebooms, G., Boogaerts, A., \& Legius, E. (2004). Predictive testing for hereditary non-polyposis colorectal cancer: Motivation, illness representations and short-term psychological impact. Patient Education and Counseling, 55, 265-274. doi:10.1016/j.pec.2003.11.002 
Claes, E., Denayer, L., Evers-Kiebooms, G., Boogaerts, A., Philippe, K., Tejpar, S., ... Legius, E. (2005). Predictive testing for Hereditary Nonpolyposis Colorectal Cancer: Subjective perception regarding colorectal and endometrial cancer, distress, and health-related behavior at one year post-test. Genetic Testing, 9, 5465. doi:10.1089/gte.2005.9.54

Claes, E., Evers-Kiebooms, G., Denayer, L., Decruyenaere, M., Boogaerts, A., Philippe, K., \& Legius, E. (2005). Predictive genetic testing for hereditary breast and ovarian cancer: Psychological distress and illness representations 1 year following disclosure. Journal of Genetic Counselling, 14, 349-363. doi: 10.1007/s10897$005-1371-4$

Clarke, S., Butler, K., \& Esplen, M. J. (2008). The phases of disclosing BRCA1/2 genetic test results to offspring. Psychooncology, 17, 797-803. doi: 10.1002/pon.1344

Collins, V., Halliday, J., Warren, R., \& Williamson, R. (2000). Cancer worries, risk perceptions and associations with interest in DNA testing and clinic satisfaction in a familial colorectal cancer clinic. Clinical Genetics, 58, 460-468. doi: 10.1034/j.1399-0004.2000.580606.x

Collins, V. R., Meiser, B., Ukoumunne, O. C., Gaff, C., St. John, D. J., \& Halliday, J. L. (2007). The impact of predictive genetic testing for Hereditary Nonpolyposis Colorectal Cancer: Three years after testing. Genetics in Medicine, 9, 290-297. doi:10.1097/GIM.0b013e31804b45db

Collins, W. A., \& Russell, G. (1991). Mother-child and father-child relationships in middle childhood and adolescence: A developmental analysis. Developmental Review, 11, 99-136. doi: 10.1016/0273-2297(91)90004-8 
Conroy, R. M., Smyth, O., Siriwardena, R., \& Fernandes, P. (1999). Health anxiety and characteristics of self-initiated general practitioner consultations. Journal of Psychosomatic Research, 46, 45-50. doi:10.1016/S0022-3999(98)00066-X

Cowan, P. A., Cowan, C. P., \& Kerig, P. K. (1993). Mothers, fathers, sons, and daughters: Gender differences in family formation and parenting style. Family, self, and society: Toward a new agenda for family research. (pp. 165-195) Lawrence Erlbaum Associates, Inc, Hillsdale, NJ.

Craig, L. (2006). Children and the revolution: A time-diary analysis of the impact of motherhood on daily workload. Journal of Sociology, 42, 125-143. doi:http://dx.doi.org/10.1177/1440783306064942

D’ Agincourt-Canning, L. (2005). The effect of experiential knowledge on construction of risk perception in Hereditary Breast/Ovarian Cancer. Journal of Genetic Counseling, 14, 55-69. doi:10.1007/s10897-005-1500-0

Deacon, B., Lickel, J., \& Abramowitz, J. S. (2008). Medical utilization across anxiety disorders. Anxiety Disorders, 22, 334-350. doi:10.1016/j.janxdis.2007.03.004.

Deimling, G. T., Bowman, K. F., Sterns, S., Wagner, L. J., \& Kahana, B. (2005). Cancerrelated health worries and psychological distress among older adult, long-term cancer survivors. Psycho-Oncology, 15, 306-320. doi:10.1002/pon.955

De Jong, A. E., Morreau, H., van Puijenbroek, M., Eilers, P. H., Wijnen, J., Nagengast, F. M., Griffioen, G., Cats, A., Menko, F. H., \& Kleibeuker, J. H. (2004). The role of mismatch repair gene defects in the development of adenomas in patients with HNPCC. Gastroenterology, 126, 42-48. doi: http://dx.doi.org/10.1053/j.gastro.2003.10.043 
Dopson, L. (2010). Role of pain management programmes in chronic pain. Nursing Standard, 25, 35-40. doi: http://dx.doi.org/10.7748/ns2010.12.25.13.35.c8120

Endler, N. S., Corace, K. M., Summerfeldt, L. J., Johnson, J. M., \& Rothbart, P. (2003). Coping with chronic pain. Personality and Individual Differences, 34, 323-346. doi:http://dx.doi.org/10.1016/S0191-8869(02)00048-X

Ernst, J., Götze, H., Brähler, E., Körner, A., \& Hinz, A. (2012). Quality of life of parents diagnosed with cancer: change over time and influencing factors. European Journal of Cancer Care, 21, 535-541. doi:10.1111/j.1365-2354.2012.01330.x

Endler, N. S., Parker, J. D. A., \& Summerfeldt, L. J. (1998). Coping with health problems: Developing a reliable and valid multidimensional measure. Psychological Assessment, 10, 195-205. doi:10.1037/1040-3590.10.3.195

Esplen, M. J., Madlensky, L., Butler, K., McKinnon, W., Bapat, B., Wong, J., ... Gallinger, S. (2001). Motivations and psychosocial impact of genetic testing for HNPCC. American Journal of Medical Genetics, 103, 9-15. doi:10.1002/ajmg.1493

Esplen, M. J., Madlensky, L., Aronson, M., Rothenmund, H., Gallinger, S., Butler, K., ... \& McLaughlin, J. (2007). Colorectal cancer survivors undergoing genetic testing for Hereditary Non-Polyposis Colorectal Cancer: Motivational factors and psychosocial functioning. Clinical Genetics, 72, 394-401. doi: 10.1111/j.13990004.2007.00893.x

Esplen, M. J., Stuckless, N., Gallinger, S., Aronson, M., Rothenmund, H., Semotiuk, K., ... \& Wong, J. (2011). Development and validation of an instrument to measure the impact of genetic testing on self-concept in Lynch syndrome. Clinical genetics, 80, 
415-423. doi: 10.1111/j.1399-0004.2011.01770.x

Esplen, M. J., Stuckless, N., Hunter, J., Liede, A., Metcalfe, K., Glendon, G., ... Irwin, E. (2009). The BRCA Self-Concept Scale: A new instrument to measure selfconcept in BRCA1/2 mutation carriers. Psycho-Oncology, 18, 1216-1229. doi:10.1002/pon. 1498

Esplen, M. J., Urquhart, C., Butler, K., Gallinger, S., Aronson, M., \& Wong, J. (2003). The experience of loss and anticipation of distress in colorectal cancer patients undergoing genetic testing. Journal of Psychosomatic Research, 55, 427-435. doi:10.1016/S0022-3999(03)00511-7

Faul, F., Erdfelder, E., Lang, A.G. \& Buchner, A. (2007). G*Power 3: A flexible statistical power analysis program for the social, behavioral, and biomedical sciences. Behavior Research Methods, 39, 175-191. doi: 10.3758/BF03193146

Fink, P., Ørnbøl, E., \& Christensen, K. S. (2010). The outcome of health anxiety in primary care. A two-year follow-up study on health care costs and self-rated health. Behavior Research Methods, 39, 175-191.

doi:10.1371/journal.pone.0009873

Fleet, R. P., Dupuis, G., Marchand, A., Burelle, D., Arsenault, A. \& Beitman, B. D. (1996). Panic disorder in emergency department chest pain patients: Prevalence, comorbidity, suicidal ideation, and physician recognition. The American Journal of Medicine, 101, 371-380. doi: 10.1016/S0002-9343(96)00224-0

Foa, E. B. (2006). Psychosocial therapy for posttraumatic stress disorder. Journal of Clinical Psychiatry, 67, 40-45. 
Folkman, S., \& Lazarus, R. S. (1990). Coping and emotion. In N. L. Stein, B. Leventhal, \& T. R. Trabasso (Eds.), Psychological and Biological Approaches to Emotion (pp. 313-332). Hillsdale, New Jersey: Lawrence Erlbaum Associates.

Furer, P., Walker, J. R., \& Freeston, M. H. (2001). Approach to integrated cognitivebehaviour therapy for intense illness worries. In G. J. G. Asmundson, S. Taylor, \& B. J. Cox (Eds.), Health Anxiety: Clinical and Research Perspectives on Hypochondriasis and Related Conditions (pp. 161-192). Chichester, United Kingdom: Wiley.

Gaff, C. L., Lynch, E., \& Spencer, L. (2006). Predictive testing of eighteen year olds: Counseling challenges. Journal of Genetic Counseling, 15, 245-251. doi:http://dx.doi.org/10.1007/s10897-006-9028-5

Ganz, P. A., Desmond, K. A., Leedham, B., Rowland, J. H, Meyerowitz, B. E., \& Belin, T. R. (2002). Quality of life in long-term, disease-free survivors of breast cancer: A follow-up study. Journal of the National Cancer Institute, 94, 39-49. doi: 10.1093/jnci/94.1.1-a

Grassi, L., Rossi, E., Sabato, S., Cruciani, G., \& Zambelli, M. (2004). Diagnostic criteria for psychosomatic research and psychosocial variables in breast cancer patients. Psychosomatics, 45, 483-491. doi:10.1176/appi.psy.45.6.483

Greeven, A., van Balkom, A. J. L. M., Visser, S., Merkelbach, J. W., van Rood, Y. R., van Dyck, R., ... Spinhoven, P. (2007). Cognitive behavior therapy and paroxetine in the treatment of hypochondriasis: A randomized controlled trial. American Journal of Psychiatry, 164, 91-99. doi:10.1176/appi.ajp.164.1.91 
Gritz, E. R., Peterson, S. K., Vernon, S. W., Marani, S. K., Baile, W. F., Watts, B. G., ... Lynch, P. M. (2005). Psychological impact of genetic testing for Hereditary Nonpolyposis Colorectal Cancer. Journal of Clinical Oncology, 23, 1902-1910. doi:10.1200/JCO.2005.07.102

Guttman, N., Zimmerman, D. R., \& Nelson, M. S. (2003). The many faces of access: Reasons for medically nonurgent emergency department visits. Journal of Health Politics Policy and Law, 23, 1089-1120. doi: 10.1215/03616878-28-6-1089

Hadjistavropoulos, H. D., Asmundson, G. J. G., LaChapelle, D. L., \& Quine, A. (2002). The role of health anxiety among patients with chronic pain in determining response to therapy. Pain research \& management: The journal of the Canadian Pain Society, 7, 127-133.

Hadjistavropoulos, H. D., Craig, K. D., \& Hadjistavropoulos, T. (1998). Cognitive and behavioral responses to illness information: the role of health anxiety. Behaviour Research and Therapy, 36, 149-164. doi: 10.1016/S0005-7967(98)00014-X

Hadjistavropoulos, H.D., Hadjistavropoulos, T., \& Quine, A. (2000). Health anxiety moderates the effects of distraction versus attention to pain. Behavior Research and Therapy, 38, 425-438. doi: 10.1016/S0005-7967(99)00044-3

Hadjistavropoulos, H., Janzen, J., Kehler, M., Leclerc, J., Sharpe, D., \& BourgaultFagnou, M. (2012). Core cognitions related to health anxiety in self-reported medical and non-medical samples. Journal of Behavioral Medicine, 35, 167-178. doi:10.1007/s10865-011-9339-3

Hadley, D. W., Jenkins, J., Dimond, E., Nakahara, K., Grogan, L., Liewehr, D. J., ... Kirsch, I. (2003). Genetic counseling and testing in families with Hereditary 
Nonpolyposis Colorectal Cancer. Archives of Internal Medicine, 163, 573-582. doi:10.1001/archinte.163.5.573

Haenen, M. A., de Jong, P. J., Schmidt, J. M., Stevens, S., \& Visser, L. (2000).

Hypochondriacs' estimation of negative outcomes: Domain-specificity and responsiveness to reassuring and alarming information. Behavior Research and

Therapy, 38, 819-833. doi: 10.1016/S0005-7967(99)00128-X

Hallowell, N., Foster, C., Eeles, R., Ardern-Jones, A., \& Watson, M. (2004).

Accommodating risk: Responses to BRCA1/2 genetic testing of women who have had cancer. Social Science \& Medicine, 59, 553-565.

doi:10.1016/j.socscimed.2003.11.025

Hampel, H., Frankel, W. L., Martin, E., Arnold, M., Khanduja, K., Kuebler, P., ... De La Chapelle, A. (2008). Feasibility of screening for Lynch syndrome among patients with colorectal cancer. Journal of Clinical Oncology, 26, 5783-5788.

doi:10.1200/JCO.2008.17.5950

Hedman, E., Andersson, G., Andersson, E., Ljótsson, B., Rück, C., Asmundson, G. J. G., \& Lindefors, N. (2011). Internet-based cognitive-behavioural therapy for severe health anxiety: Randomised controlled trial. The British Journal of Psychiatry, 198, 230-236. doi:10.1192/bjp.bp.110.086843

Henderson, A. S., Montgomery, I. M., \& Williams, C. L. (1972). Psychological immunization: A proposal for preventative psychiatry. Lancet, 1, 111-1113. doi: $10.1016 / \mathrm{S} 0140-6736(72) 91441-9$

Hitchcock, P.B., \& Mathews, A. (1992). Interpretation of bodily symptoms in 
hypochondriasis. Behavior Research and Therapy, 30, 223-234. doi:

$10.1016 / 0005-7967(92) 90068-\mathrm{R}$

Hsieh, H. F., \& Shannon, S. S. (2005). Three approaches to qualitative content analysis. Qualitative Health Research, 15, 1277-1288. doi: 10.1177/1049732305276687

Janzen, J. A., \& Hadjistavropoulos, H. D. (2008). Examination of Negative Affective Responses to waiting for surgery. Canadian Journal of Nursing Research, 40, $72-$ 91.

Järvinen, H. J., Renkonen-Sinisalo, L., Aktán-Collán, K., Peltomäki, P., Aaltonen, L. A., \& Mecklin, J.-P. (2009). Ten years after mutation testing for Lynch syndrome: Cancer incidence and outcome in mutation-positive and mutation-negative family members. Journal of Clinical Oncology, 27, 4793-4797.

doi:10.1200/JCO.2009.23.7784

Järvinen, H. J., Aarnio, M., Mustonen, H., Aktan-Collan, K., Aaltonen, L. A., Peltomäki, P., ... Mecklin, J. (2000). Controlled 15-year trial on screening for colorectal cancer in families with hereditary nonpolyposis colorectal cancer. Gastroenterology, 118, 829-834. doi:10.1016/S0016-5085(00)70168-5

Jemal, A., Bray, F., Center, M. M., Ferlay, J., Ward, E., \& Forman, D. (2011). Global cancer statistics. CA: A Cancer Journal for Clinicians, 61, 69-90. doi:10.3322/caac.20107

Jenkins, M. A., Baglietto, L., Dowty, J. G., Van Vliet, C. M., Smith, L., Mead, L. J., ... Southey, M. C. (2006). Cancer risks for mismatch repair gene mutation carriers: A population-based early onset case-family study. Clinical Gastroenterology and Hepatology, 4, 489-498. doi:10.1016/j.cgh.2006.01.002 
Johnson, J. M., \& Endler, N. S. (2002). Coping with human immunodeficiency virus: Do optimists fare better? Current Psychology: A Journal for Diverse Perspectives on Diverse Psychological Issues, 21, 3-16. doi: 10.1007/BF02903156

Jones, S. L., Hadjistavropoulos, H. D., \& Sherry, S. B. (2012). Health anxiety in women with early-stage breast cancer: What is the relationship to social support? Canadian Journal of Behavioural Science, 44, 108-116. doi: 10.1037/a0027526

Kehler, M., \& Hadjistavropoulos, H. (2009). Is health anxiety a significant problem for individuals with multiple sclerosis? Journal of Behavioral Medicine, 32, 150-161. doi:10.1007/s10865-008-9186-Z

Keller, M., Jost, R., Haunstetter, C. M., Kienle, P., Knaebel, H.-P., Gebert, J., ... Mazitschek, U. (2002). Comprehensive genetic counseling for families at risk for HNPCC: Impact on distress and perceptions. Genetic Testing, 6, 291-302. doi:10.1089/10906570260471822

Keller, M., Jost, R., Haunstetter, C. M., Sattel, H., Schroeter, C., Bertsch, U., ... Brechtel, A. (2008). Psychosocial outcome following genetic risk counselling for familial colorectal cancer: A comparison of affected patients and family members. Clinical Genetics, 74, 414-424. doi:10.1111/j.1399-0004.2008.01089.x

Kenen, R., Ardern-Jones, A., \& Eeles, R. (2006). “Social separation” among women under 40 years of age diagnosed with breast cancer and carrying a BRCA1 or BRCA2 mutation. Journal of Genetic Counseling, 15, 149-162. doi: $10.1007 / \mathrm{s} 10897-005-9015-2$

Kennedy, B. L., \& Schwab, J. J. (1997). Utilization of medical specialists by anxiety disorder patients. Psychosomatics, 38, 112-190. doi: 10.1016/S0033- 
$3182(97) 71478-6$

Klahr, A. M., \& Burt, S. A. (2014). Elucidating the etiology of individual differences in parenting: A meta-analysis of behavioral genetic research. Psychological Bulletin, 140(2), 544-586. doi:http://dx.doi.org/10.1037/a0034205

Kirby, S. E., \& Yardley, L. (2009). The contribution of symptoms of posttraumatic stress disorder, health anxiety and intolerance of uncertainty to distress in Meniere's disease. The Journal of Nervous and Mental Disease, 197, 324-329. doi: 10.1097/NMD.0b013e3181a20866

Koch, L., Jansen, L., Brenner, H. \& Arndt, V. (2013). Fear of recurrence and disease progression in long-term ( $\geq 5$ years) cancer survivors: A systematic review of quantitative studies. Psycho-Oncology, 22, 1-11. doi: 10.1002/pon.3022

Koehly, L. M., Peterson, S. K., Watts, B. G., Kempf, K. K., Vernon, S. W., \& Gritz, E. R. (2003). A social network analysis of communication about Hereditary Nonpolyposis Colorectal Cancer genetic testing and family functioning. Cancer Epidemiology Biomarkers \& Prevention, 12, 304-313.

Köteles, F., Freyler, A., Kökönyei, G., \& Bárdos, G. (2014). Family background of modern health worries, somatosensory amplification, and health anxiety: A questionnaire study. Journal of health psychology. Retrieved from http://hpq.sagepub.com/content/early/2014/01/07/1359105313516661.abstract. doi: $10.1177 / 1359105313516661$

Krauß, O., Ernst, J., Kuchenbecker, D., Hinz, A., \& Schwarz, R. (2007). Predictors of mental disorders in patients with malignant diseases: Empirical results. 
Psychotherapie Psychosomatik Medizinische Psychologie, 57, 273-280. doi:10.1055/s-2006-952034

Lancaster, J. M., Carney, M. E., \& Futreal, P. A. (1997). BRCA 1 and 2--A genetic link to familial breast and ovarian cancer. Medscape Women's Health, 2, 7.

Landsbergen, K. M., Prins, J. B., Brunner, H. G., van Duijvendijk, P., Nagengast, F. M., van Krieken, J. H., ... \& Hoogerbrugge, N. (2012). Psychological distress in newly diagnosed colorectal cancer patients following microsatellite instability testing for Lynch syndrome on the pathologist's initiative. Familial Cancer, 11, 259-267. doi: 10.1007/s10689-012-9510-1

Lau, R. R., Quadrel, M. J., \& Hartman, K. A. (1990). Development and change of young adults' preventive health beliefs and behavior: Influence from parents and peers. Journal of health and social behavior, 31, 240-259. doi: 10.2307/2136890

Lega, F., \& Mengoni, A. (2008). Why non-urgent patients choose emergency over primary care services? Empirical evidence and managerial implications. Health Policy, 88, 326-338. doi: 10.1016/j.healthpol.2008.04.005

Lerman, C., \& Croyle, R. (1994). Psychological issues in genetic testing for breast cancer susceptibility. Archives of Internal Medicine, 154, 609-616.

doi:10.1001/archinte.1994.00420060027003

Lerman, C., Croyle, R. T., Tercyak, K. P., \& Hamann, H. (2002). Genetic testing: Psychological aspects and implications. Journal of Consulting and Clinical Psychology, 70, 784-797. doi:10.1037/0022-006X.70.3.784

Lerman, C., Marshall, J., Audrain, J., \& Gomez-Caminero, A. (1998). Genetic testing for colon cancer susceptibility: Anticipated reactions of patients and challenges to 
providers. International Journal of Cancer, 69, 58-61. doi:10.1002/(SICI)10970215(19960220)69:1<58::AID-IJC15>3.0.CO;2-G

Lewis, L. M., Alpert, E. N., Lebo, B. L., \& Bane, G. A. (2006). Comparison of demographic, clinical, and attitudinal factors between urgent and nonurgent emergency department users. Academic Emergency Medicine, 13, 131. doi: 10.1016/j.healthpol.2008.04.005

Lim, J., Macluran, M., Price, M., Bennett, B., Butow, P., \& kConFab Psychosocial Group. (2004). Short- and long-term impact of receiving genetic mutation results in women at increased risk for hereditary breast cancer. Journal of Genetic Counseling, 13, 115-133. doi:10.1023/B:JOGC.0000018822.56297.a6

Lindor, N. M., Petersen, G. M., Hadley, D. W., Kinney, A. Y., Miesfeldt, S., Lu, K. H., ... Press, N. (2006). Recommendations for the care of individuals sith an inherited predisposition to Lynch syndrome. JAMA: The Journal of the American Medical Association, 296, 1507-1517. doi:10.1001/jama.296.12.1507

Lu, K. H. \& Broaddus, R. R. (2005). Gynaecologic cancers in Lynch syndrome/HNPCC. Familial Cancer, 4, 249-254. doi: 10.1007/s10689-005-1838-3

Lynch, H. T., Lynch, P. M., Lanspa, S. J., Snyder, C. L., Lynch, J. F., \& Boland, C. R. (2009). Review of the Lynch syndrome: History, molecular genetics, screening, differential diagnosis, and medicolegal ramifications. Clinical Genetics, 76, 1-18. doi:10.1111/j.1399-0004.2009.01230.x

Lynch, H. T., Lemon, S. J., Durham, C., Tinley, S. T., Connolly, C., Lynch, J. F., ... Narod, S. (1997). A descriptive study of BRCA1 testing and reactions to 
disclosure of test results. Cancer, 79, 2219-2228. doi:10.1002/(SICI)10970142(19970601)79:11<2219::AID-CNCR21>3.0.CO;2-Y

MacDonald, D. J., Sarna, L., Weitzel, J. N., et al. (2010). Women's perceptions of the personal and family impact of genetic cancer risk assessment: Focus group findings. Journal Genetic Counseling, 19, 148-160. doi: 10.1007/s10897-0099267-3

Macrodimitris, S. D., \& Endler, N. S. (2001). Coping, control, and adjustment in type 2 diabetes. Health Psychology, 20, 208-216. doi:10.1037/0278-6133.20.3.208

Maloney, E., Edgerson, S., Robson, M., Offit, K., Brown, R., Bylund, C., \& Kissane, D. W. (2012). What women with breast cancer discuss with clinicians about risk for their adolescent daughters. Journal of Psychosocial Oncology, 30, 484. doi: $10.1080 / 07347332.2012 .684855$

Marcus, D.K. (1999). The cognitive-behavioral model of hypochondriasis: Misinformation and triggers. Journal of Psychosomatic Research, 47, 79-91. doi: $10.1016 / \mathrm{S} 0022-3999(99) 00008-2$

Marcus, D.K., \& Church, S. E. (2003). Are dysfunctional beliefs about illness unique to hypochondriasis? Journal of Psychosomatic Research, 54, 543-547. doi: $10.1016 / \mathrm{S} 0022-3999(02) 00526-3$

Marcus, D. K., Hughes, K. T., \& Arnau, R. C. (2008). Health anxiety, rumination, and negative affect: A mediational analysis. Journal of Psychosomatic Research, 64, 495-501. doi: http://dx.doi.org/10.1016/j.jpsychores.2008.02.004 
Martin, A., \& Jacobi, F. (2006). Features of hypochondriasis and illness worry in the general population in Germany. Psychosomatic medicine, 68, 770-777. doi: 10.1097/01.psy.0000238213.04984.b0

Mcallister, M. (2002). Predictive genetic testing and beyond: A theory of engagement. Journal of Health Psychology, 7, 491-508. doi:10.1177/1359105302007005628

McInerney-Leo, A., Biesecker, B. B., Hadley, D. W., Kase, R. G., Giambarresi, T. R., Johnson, E., ... \& Struewing, J. P. (2005). BRCA1/2 testing in hereditary breast and ovarian cancer families II: Impact on relationships. American Journal of Medical Genetics Part A, 133, 165-169. doi: 10.1002/ajmg.a.30566

Meiser, B., Collins, V., Warren, R., Gaff, C., St John, D. J. B., Young, M., ... Halliday, J. (2004). Psychological impact of genetic testing for Hereditary Non-Polyposis Colorectal Cancer. Clinical Genetics, 66, 502-511. doi:10.1111/j.13990004.2004.00339.x

Meiser, B. (2005). Psychological impact of genetic testing for cancer susceptibility: An update of the literature. Psycho-Oncology, 14, 1060-1074. doi:10.1002/pon.933

Meiser, B. \& Halliday, J. L. (2002). What is the impact of genetic counselling in women at increased risk of developing hereditary breast cancer? A meta-analytic review. Social Science \& Medicine, 54, 1463-1470. doi:10.1016/S0277-9536(01)00133-2

Miesfeldt, S., Cohn, W. F., \& Jones, S. M. (2003). Breast cancer survivors' attitudes towards communication of breast cancer risk to their children. American Journal Medicine Genetics C Seminars in Medical Genetics, 119C, 45-50. 10.1002/ajmg.c.10012 
Mitchell, R. J., Farrington, S. M., Dunlop, M. G., \& Campbell, H. (2002). Mismatch repair genes hMLH1 and hMSH2 and colorectal cancer: A HuGE Review. American Journal of Epidemiology, 156, 885-902. doi:10.1093/aje/kwf139

Moorey, S., Greer, S., Watson, M., Gorman, C., Rowden, L., Tunmore, R., ... Bliss, J. (1991). The factor structure and factor stability of the Hospital Anxiety and Depression Scale in patients with cancer. The British Journal of Psychiatry, 158, 255-259. doi:10.1192/bjp.158.2.255

Möslein, G., Krause-Paulus, R., Hegger, R., Peterschulte, G., \& Vogel, T. (2000). Clinical aspects of Hereditary Nonpolyposis Colorectal Cancer. Annals of the New York Academy of Sciences, 910, 75-84. doi:10.1111/j.17496632.2000.tb06702.x

Murakami, Y., Okamura, H., Sugano, K., Yoshida, T., Kazuma, K., Akechi, T., \& Uchitomi, Y. (2004). Psychologic distress after disclosure of genetic test results regarding hereditary nonpolyposis colorectal carcinoma. Cancer, 101, 395-403. doi:10.1002/cncr.20363

Nakao, M., Shinozaki, Y., Ahern, D. K., \& Barsky, A. J. (2011). Anxiety as a predictor of improvements in somatic symptoms and health anxiety associated with cognitivebehavioral intervention in hypochondriasis. Psychotherapy and psychosomatics, 80(3), 151-158. doi: 10.1159/000320122

Norum, J. (2000). Health, life and disability insurance and hereditary risk for breast or colorectal cancer. Acta Oncologica, 39, 189-193. doi: 10.1080/028418600430752

Owens, K., \& Antony, M. (2011). Overcoming Health Anxiety: Letting Go of Your Fear of Illness. Oakland, CA: New Harbinger Publications. 
Owens, K. M. B., Asmundson, G. J. G, Hadjistavropoulos, T., \& Owens, T. J. (2004). Attentional bias toward illness threat in individuals with elevated health anxiety. Cognitive Therapy and Research, 28, 57-66. doi:

10.1023/B:COTR.0000016930.85884.29

Palmquist, A. E., Koehly, L. M., Peterson, S. K., Shegog, M., Vernon, S. W., \& Gritz, E. R. (2010). "The cancer bond": Exploring the formation of cancer risk perception in families with Lynch syndrome. Journal of Genetic Counselling, 19, 473-486. doi: $10.1007 / \mathrm{s} 10897-010-9299-8$

Patenaude, A. F., Dorval, M., DiGianni, L. S., Schneider, K. A., Chittenden, A., \& Garber, J. E. (2006). Sharing BRCA1/2 test results with first-degree relatives: Factors predicting who women tell. Journal of Clinical Oncology, 24, 700-706. doi:10.1200/JCO.2005.01.7541

Payne, K., Binik, Y., Pukall, C., Thaler, L., Amsel, R., \& Khalifé, S. (2007). Effects of sexual arousal on genital and non-genital sensation: A comparison of women with Vulvar Vestibulitis Syndrome and healthy controls. Archives of Sexual Behavior, 36, 289-300. doi:10.1007/s10508-006-9089-4

Peshkin, B. N., Demarco, T. A., \& Tercyak, K. P. (2010). On the development of a decision support intervention for mothers undergoing BRCA1/2 cancer genetic testing regarding communicating test results to their children. Familial Cancer, 9, 89-97. doi: 10.1007/s 10689-009-9267-3 
Peterson, H. V., Esplen, M. J., Ladelund, S., Bernstein, I., Sunde, L., Carlsson, C., \& Nilbert, M. (2011). Limited impact on self-concept in individuals with Lynch syndrome: Results from a national cohort study. Familial Cancer, 10, 633-639. doi: 10.1007/s10689-011-9459-5

Pugh, N. E., Hadjistavropoulos, H. D., \& Sharpe, D. (2012). The Reassurance Questionnaire: Comparison of the latent structure in university, community, and medical samples. Psychology, Health \& Medicine, 18, 117-124. doi:

$10.1080 / 13548506.2012 .668196$

Ratcliffe, D., Macleod, A., \& Sensky, T. (2006). Anxiety in patients who have had a myocardial infarction: The maintaining role of perceived physical sensations and causal attributions. Behavioural and Cognitive Psychotherapy, 34, 201-217. doi: $10.1017 / \mathrm{S} 1352465806002773$

Rief, W., Hiller, W., \& Margraf, J. (1998). Cognitive aspects of hypochondriasis and the somatization syndrome. Journal of Abnormal Psychology, 107, 587-95. doi: 10.1037/0021-843X.107.4.587

Rimes, K. A., Salkovskis, P. M., Jones, L., \& Lucassen, A. M. (2006). Applying a cognitive behavioral model of health anxiety in a cancer genetics service. Health Psychology, 25, 171-180. doi:10.1037/0278-6133.25.2.171

Ritter, P. L., Stewart, A. L., Kaymaz, H., Sobel, D. S., Block, D. A., \& Lorig, K. R. (2001). Self-reports of health care utilization compared to provider records. Journal of Clinical Epidemiology, 54, 136-141. doi: 10.1016/S08954356(00)00261-4 
Rode, S., Salkovskis, P., Dowd, H., \& Hanna, M. (2006). Health anxiety levels in chronic pain clinic attenders. Journal of Psychosomatic Research, 60, 155-161. doi:10.1016/j.jpsychores.2005.07.005

Rodriguez-Bigas, M. A., Vasen, H. F., O'Malley, L., Rosenblatt, M. J., Farrell, C., Weber, T. K., \& Petrelli, N. J. (1998). Health, life, and disability insurance and hereditary nonpolyposis colorectal cancer. American journal of human genetics, 62, 736. doi: $10.1086 / 301753$

Rolland, J. S., \& Williams, J. K. (2005). Toward a biopsychosocial model for 21stCentury genetics. Family Process, 44, 3-24. doi:10.1111/j.15455300.2005.00039.x

Salkovskis, P M, Rimes, K. A., Warwick, H. M. C., \& Clark, D. M. (2002). The Health Anxiety Inventory: Development and validation of scales for the measurement of health anxiety and hypochondriasis. Psychological Medicine, 32, 843-853.

Salkovskis, P. M., \& Warwick, H. M. (2001). Meaning, misinterpretations, and medicine: A cognitive-behavioral approach to understanding health anxiety and hypochondriasis. In Starcevic, V. and Lipsitt, D. R. (eds.), Hypochondriasis: Modern Perspectives on an Ancient Malady (pp. 202-222). New York, NY: Oxford University Press

Salkovskis, Paul M., \& Warwick, H. M. C. (1986). Morbid preoccupations, health anxiety and reassurance: a cognitive-behavioural approach to hypochondriasis. Behaviour Research and Therapy, 24, 597-602. doi:10.1016/00057967(86)90041-0 
Seivewright, H., Salkovskis, P., Green, J., Mullan, N., Behr, G., Carlin, E., ... Tyrer, P. (2004). Prevalence and service implications of health anxiety in genitourinary medicine clinics. International Journal of STD \& AIDS, 15, 519-522. doi:10.1258/0956462041558122

Seivewright, H., Green, J., Salkovskis, P., Barrett, B., Nur, U., \& Tyrer, P. (2008). Cognitive-behavioural therapy for health anxiety in a genitourinary medicine clinic: Randomised controlled trial. The British Journal of Psychiatry, 193, 332 337. doi:10.1192/bjp.bp.108.052936

Shiloh, S., Drori, E., Orr-urtreger, A., \& Friedman, E. (2009). Being 'at-risk' for developing cancer: Cognitive representations and psychological outcomes. Journal of Behavioral Medicine, 32, 197-208. doi: http://dx.doi.org/10.1007/s10865-0089178-Z

Siegel, R., Naishadham, D., \& Jemal, A. (2012). Cancer statistics, 2012. CA: A Cancer Journal for Clinicians, 62, 10-29. doi:10.3322/caac.20138

Sørensen, P., Birket-Smith, M., Wattar, U., Buemann, I., \& Salkovskis, P. (2011). A randomized clinical trial of cognitive behavioural therapy versus short-term psychodynamic psychotherapy versus no intervention for patients with hypochondriasis. Psychological Medicine, 41, 431-441. doi: 10.1017/S0033291710000292.

Stark, D. P. H., \& House, A. (2000). Anxiety in cancer patients. British Journal of Cancer, 83, 1261-1267. doi: 10.1054\%2Fbjoc.2000.1405 
Stark, D., Kiely, M., Smith, A., Morley, S., Selby, P., \& House, A. (2004). Reassurance and the anxious cancer patient. British Journal of Cancer, 91, 893-899. doi: 10.1038/sj.bjc.6602077

Stemler, S. E. (2004). A comparison of consensus, consistency, and measurement approaches to estimating interrater reliability. Practical Assessment, Research \& Evaluation, 9. Retrieved February 9, 2014 from http://PAREonline.net/getvn.asp?v=9\&n=4.

Sunderland, M., Newby, J. M., \& Andrews, G. (2012). Health anxiety in Australia: Prevalence, comorbidity, disability and service use. The British Journal of Psychiatry, 202, 56-61. doi:10.1192/bjp.bp.111.103960

Tabachnick, B., \& Fidell, L. (2001). Using Multivariate Statistics (4th ed.). Boston: Allyn $\&$ Bacon.

Taylor, S., \& Asmundson, G. J. G. (2004). Treating Health Anxiety: A CognitiveBehavioral Approach. New York: NY. Guilford Press.

Taylor, S., Jang, K. L., Stein, M. B., \& Asmundson, G. J. (2008). A behavioral-genetic analysis of health anxiety: Implications for the cognitive-behavioral model of hypochondriasis. Journal of Cognitive Psychotherapy, 22, 143-153. doi: $10.1891 / 0889-8391.22 .2 .143$

Taylor, S., Thordarson, D. S., Jang, K. L., \& Asmundson, G. J. (2006). Genetic and environmental origins of health anxiety: A twin study. World Psychiatry, 5, 4750.

Tercyak, K. P., Hughes, C., Main, D., Snyder, C., Lynch, J. F., Lynch, H. T., \& Lerman, C. (2001). Parental communication of BRCA1/2 genetic test results to children. 
Patient Education and Counseling, 42, 213-224. doi: 10.1016/S07383991(00)00122-1

Tercyak, K. P., Peshkin, B. N., DeMarco, T. A., Brogan, B. M., \& Lerman, C. (2002). Parent-child factors and their effect on communicating BRCA1/2 test results to children. Patient Education and Counseling, 47(2), 145-153. doi: 10.1016/S07383991(01)00192-6

Tercyak, K. P., Peshkin, B. N., DeMarco, T. A., Farkas Patenaude, A., Schneider, K. A., Garber, J. E., ... \& Schwartz, M. D. (2007). Information needs of mothers regarding communicating BRCA1/2 cancer genetic test results to their children. Genetic testing, 11, 249-255. doi:10.1089/gte.2006.0534.

Tilburt, J. C., James, K. M., Sinicrope, P. S., Eton, D. T., Costello, B. A., Carey, J., ... Murad, M. H. (2011). Factors influencing cancer risk perception in high risk populations: A systematic review. Hereditary Cancer in Clinical Practice, 9, 2. doi:10.1186/1897-4287-9-2

Trask, P. C., Paterson, A. G., Wang, C., Hayasaka, S., Milliron, K. J., Blumberg, L. R., ... Merajver, S. D. (2001). Cancer-specific worry interference in women attending a breast and ovarian cancer risk evaluation program: Impact on emotional distress and health functioning. Psycho-Oncology, 10, 349-360. doi:10.1002/pon.510

Trepanier, A., Ahrens, M., McKinnon, W., Peters, J., Stopfer, J., Grumet, S., ... Vockley, C. (2004). Genetic cancer risk assessment and counseling: Recommendations of the National Society of Genetic Counselors. Journal of Genetic Counseling, 13, 83-114. doi:10.1023/B:JOGC.0000018821.48330.77 
Tyrer, H. (2013). Tackling Health Anxiety: A CBT Handbook. London: UK. RCPsych Publications.

Vadaparampil, S. T., Ropka, M., \& Stefanek, M. E. (2005). Measurement of psychological factors associated with genetic testing for hereditary breast, ovarian and colon cancers. Familial Cancer, 4, 195-206. doi:10.1007/s10689-004-1446-7

van Oostrom, I., Meijers-Heijboer, H., Duivenvoorden, H. J., Bröcker-Vriends, Annette H. J. T., van Asperen, C. J., Sijmons, R. H., . . Tibben, A. (2007). Comparison of individuals opting for BRCA1/2 or HNPCC genetic susceptibility testing with regard to coping, illness perceptions, illness experiences, family system characteristics and hereditary cancer distress. Patient Education and Counseling, 65(1), 58-68. doi:http://dx.doi.org/10.1016/j.pec.2006.05.006

Van Oostrom, I., Meijers-Heijboer, H., Duivenvoorden, H. J., Bröcker-Vriends, A. H. J. T., Van Asperen, C. J., Sijmons, R. H., ... Tibben, A. (2006). Experience of parental cancer in childhood is a risk factor for psychological distress during genetic cancer susceptibility testing. Annals of Oncology, 17, 1090-1095. doi:10.1093/annonc/md1069

Van Oostrom, Iris, Meijers-Heijboer, H., Duivenvoorden, H. J., Bröcker-Vriends, A. H. J. T., Asperen, V., J, C., ... Tibben, A. (2006). A prospective study of the impact of genetic susceptibility testing for BRCA1/2 or HNPCC on family relationships. Psycho-Oncology, 16, 320-328. doi:10.1002/pon.1062

Vasen, H. F., Blanco, I., Aktan-Collan, K., Gopie, J. P., Alonso, A., Aretz, S., ... \& Möslein, G. (2013). Revised guidelines for the clinical management of Lynch 
syndrome (HNPCC): Recommendations by a group of European experts. Gut, 62, 812-823. doi:10.1136/gutjnl-2012-304356

Vernon, S. W., Gritz, E. R., Peterson, S. K., Amos, C. I., Perz, C. A., Baile, W. F., \& Lynch, P. M. (1997). Correlates of psychologic distress in colorectal cancer patients undergoing genetic testing for hereditary colon cancer. Health Psychology, 16, 73-86. doi: 10.1037/0278-6133.16.1.73

Vriezekolk, J. E., van Lankveld, W. G., Geenen, R., van den Ende, C. H. (2011). Longitudinal association between coping and psychological distress in rheumatoid arthritis: A systematic review. Annals of the Rheumatic Diseases, 70, 1243-1250. doi:10.1136/ard.2010.143271

Wade, T. D., Nehmy, T., \& Koczwara, B. (2004). Predicting worries about health after breast cancer surgery. Psycho-Oncology, 14, 503-509. doi:10.1002/pon.866

Wagner, A., van Kessel, I., Kriege, M., Tops, C., Wijnen, J., Vasen, H., ... MeijersHeijboer, H. (2005). Long term follow-up of HNPCC gene mutation carriers: Compliance with screening and satisfaction with counseling and screening procedures. Familial Cancer, 4, 295-300. doi:10.1007/s10689-005-0658-9

Walsh, S. R., Manuel, J. C., \& Avis, N. E. (2005). The impact of breast cancer on younger women's relationships with their partner and children. Families, Systems, \& Health, 23, 80-93. doi:10.1037/1091-7527.23.1.80

Warwick, H. M. C., \& Salkovskis, P. M. (1990). Hypochondriasis. Behaviour Research and Therapy, 28, 105-117. doi:10.1016/0005-7967(90)90023-C 
Warwick, H. M., Clark, D. M., Cobb, A. M., \& Salkovskis, P. M. (1996). A controlled trial of cognitive-behavioural treatment of hypochondriasis. The British Journal of Psychiatry, 169, 189-195. doi:10.1192/bjp.169.2.189

Watson, D., Clark, L. A., \& Tellegen, A. (1988). Development and validation of brief measures of positive and negative affect: The PANAS scales. Journal of Personality and Social Psychology, 54, 1063-1070. doi: 10.1037/00223514.54.6.1063

Watson, M., Lloyd, S., Davidson, J., Meyer, L., Eeles, R., Ebbs, S., \& Murday, V. (1999). The impact of genetic counselling on risk perception and mental health in women with a family history of breast cancer. British Journal of Cancer, 79, 868874. doi:10.1038/sj.bjc.6690139

Weck, F., Neng, J. M. B., Richtberg, S., \& Stangier, U. (2012). The restrictive concept of good health in patients with hypochondriasis. Journal of Anxiety Disorders, 26, 792-798. doi:http://dx.doi.org/10.1016/j.janxdis.2012.07.001

Wenzel, L. B., Fairclough, D. L., Brady, M. J., Cella, D., Garrett, K. M., Kluhsman, B. C., et al. (1999). Age-related differences in the quality of life of breast carcinoma patients after treatment. Cancer, 86, 1768-74. doi: 10.1002/(SICI)10970142(19991101)86:9<1768::AID-CNCR19>3.0.CO;2-O

Werner-Lin, A. (2010). Building the cancer family: Family planning in the context of inherited breast and ovarian cancer risk. Journal of the Society for Social Work and Research, 1, 14-27. doi: 10.5243/jsswr.2010.3

Wilson, B. J., Forrest, K., van Teijlingen, E. R., McKee, L., Haites, N., Matthews, E., \& Simpson, S. A. (2004). Family communication about genetic risk: The little 
that is known. Public Health Genomics, 7(1), 15-24. doi: 10.1159/000080300 World Health Organization. (1992). The ICD-10 classification of mental and behavioural disorders: Clinical descriptions and diagnostic guidelines. Geneva: World Health Organization.

Zabora J., BrintzenhofeSzoc, K., Curbow, B., Hooker, C., \& Piantadosi, S. (2001). The prevalence of psychological distress by cancer site. Psycho-Oncology, 10, 19-28. doi: 10.1002/1099-1611(200101/02)10:1<19::AID-PON501>3.0.CO;2-6 Zigmond, A. S., \& Snaith, R. P. (1983). The Hospital Anxiety and Depression Scale. Acta Psychiatrica Scandinavica, 67, 361-370. doi:10.1111/j.16000447.1983.tb09716.x 